\title{
The maximum efficiency of nano heat engines depends on more than temperature
}

\author{
Mischa P. Woods ${ }^{1,2}$, Nelly Huei Ying $\mathrm{Ng}^{2,3}$, and Stephanie Wehner ${ }^{2}$ \\ ${ }^{1}$ Institute for Theoretical Physics, ETH Zurich, Switzerland \\ ${ }^{2}$ QuTech, Delft University of Technology, Lorentzweg 1, 2611 CJ Delft, Netherlands \\ ${ }^{3}$ Dahlem Center for Complex Quantum Systems, Freie Universität Berlin, 14195 Berlin, Germany
}

Sadi Carnot's theorem regarding the maximum efficiency of heat engines is considered to be of fundamental importance in thermodynamics. This theorem famously states that the maximum efficiency depends only on the temperature of the heat baths used by the engine, but not on the specific structure of baths. Here, we show that when the heat baths are finite in size, and when the engine operates in the quantum nanoregime, a revision to this statement is required. We show that one may still achieve the Carnot efficiency, when certain conditions on the bath structure are satisfied; however if that is not the case, then the maximum achievable efficiency can reduce to a value which is strictly less than Carnot. We derive the maximum efficiency for the case when one of the baths is composed of qubits. Furthermore, we show that the maximum efficiency is determined by either the standard second law of thermodynamics, analogously to the macroscopic case, or by the non increase of the max relative entropy, which is a quantity previously associated with the single shot regime in many quantum protocols. This relative entropic quantity emerges as a consequence of additional constraints, called generalized free energies, that govern thermodynamical transitions in the nanoregime. Our findings imply that in order to maximize efficiency, further considerations in choosing bath Hamiltonians should be made, when explicitly constructing quantum heat engines in the future. This understanding of thermodynamics has implications for nanoscale engineering aiming to construct small thermal machines.

\section{Introduction}

Nicolas Léonard Sadi Carnot is often described as the "father of thermodynamics". In his only publication in 1824 [1], Carnot gave the first successful theory in analysing the maximum efficiency of heat engines. It was later used by Rudolf Clausius and Lord Kelvin to formalize the second law of thermodynamics and define the concept of entropy [2, 3]. In particular, Carnot studied heat engines where working fluids undergo heating and cooling between two heat sources at different temperatures. In 1824 , he concluded that the maximum efficiency attainable did not depend upon the exact nature of the working fluids [1]:
The motive power of heat is independent of the agents employed to realize it; its quantity is fixed solely by the tempera- tures of the bodies between which is ef- fected, finally, the transfer of caloric.

For his "motive power of heat", we would today say "the efficiency of a reversible heat engine", and "transfer of caloric" we would replace with "reversible transfer of heat". Carnot knew intuitively that his engine would have maximum efficiency, but was unable to state what that efficiency should be. He also defined a hypothetical heat engine (now known as the Carnot engine) which would achieve the maximum efficiency. Later, this efficiency - now known as the Carnot efficiency — was shown to be

$$
\eta_{C}=1-\frac{\beta_{\text {Hot }}}{\beta_{\text {Cold }}},
$$

where $\beta_{\text {Cold }}, \beta_{\text {Hot }}$ are the inverse temperatures of the cold and hot heat baths ${ }^{1}$; as they are now more commonly referred to.

\footnotetext{
${ }^{1}$ Throughout this manuscript we set the Boltzmann's and Planck's constants, $k_{\mathrm{B}}$ and $\hbar$ to unity.
} 
Unlike the large scale heat engines that inspired thermodynamics, we are now able to build nanoscale quantum machines consisting of a mere handful of particles, and this has prompted many efforts to understand quantum thermodynamics $[4,5,6,7,8,9,10,11,12,13,14,15$, $16,17,18,19,20,21,22,23,24]$. In particular, given such nanoscale devices, one of the main issues addressed in quantum thermodynamics is the single-shot analysis of thermodynamical state transitions or work extraction. This approach addresses the scenario of moving away from the thermodynamic limit of infinitely many identical particles: one is increasingly interested in how a single copy of some small system (such as one to two atoms), in one instance of its evolution, may exhibit thermodynamical behaviour (due to interactions with its environment). These approaches are complementary to previous approaches of studying the ensemble or time averaged behaviour of the system. Many of the results in single-shot analysis have shown that the workings of thermodynamics become more intricate in such regimes $[6,7,8,9]$. While earlier efforts in quantum thermodynamics apply methods in statistical physics to average over time [25], or particular models of open systems dynamics [26, 27, 28, 29, 30, 31, 32, 33, 34, 35], single-shot thermodynamics adopts tools from quantum information theory to contribute to answering similar physical problems in a different light.

Following this approach of single-shot quantum thermodynamics, we show in this manuscript that unlike at the macroscopic scale — where Carnot's fundamental results undoubtedly hold - there are new fundamental limitations to the maximal efficiency at the nanoscale. Most significantly, we show that this maximum efficiency ${ }^{2}$ depends on the heat baths. In other words, we find that the Carnot efficiency can be achieved, but only when certain conditions on the bath Hamiltonian are satisfied. Otherwise, a reduced efficiency is obtained, highlighting the significant difference in the performance of heat engines in the single-shot regime.

\footnotetext{
${ }^{2}$ We emphasize that by "maximum efficiency", it is understood that, for fixed hot and cold bath temperatures, we are maximising the efficiency over all possible heat engine cycles, in other words any machine that may interact with the different baths and undergo a cyclic process.
}

This manuscript is organized as follows: in Section 2, we first introduce the setup, and clearly detail all assumptions made about the heat engine model of our study. Next in Section 2.2 we introduce different notions of work in the nano regime, which will be important for understanding our results. Next, in Section 3 we detail our findings. We start by showing that although a positive amount of "perfect work" cannot be extracted, some amount of "near perfect" work is possible, and we derive the efficiency that can be achieved while extracting this type of work. Finally we conclude with a summary of our results and open questions in Section 5.

\section{Setup}

\subsection{The heat engine model}

A heat engine (see Fig. 1) is a procedure for extracting work from a temperature difference between two systems. It comprises of four basic elements: the two thermal baths at distinct inverse temperatures $\beta_{\text {Hot }}$ and $\beta_{\text {Cold }}$, a machine, and a system to which work is extracted, often referred to as a battery. The machine interacts with these baths in such a way that utilizes the temperature difference between the two baths to perform work extraction. The battery is a particularly useful way of quantifying extracted work in this model as it allows for the transfer/storage of energy into the battery ancillary system, while the machine returns to its original state. Different battery models such as the work qubit or qubits $[6,7,10,36]$, the weight $[37,29]$ and the purity battery $[38,39]$ have been recently studied and used to quantify work. Although the concept resembles the notion of a work reservoir such as in [40], in these recent works, the problem of extracting work is cast in a strongly operational perspective: one is not only interested in increasing the average energy of the battery, but is also interested in the final state such a battery takes, so that it may be used in the future to enable other processes [41].

In this section, we describe a general heat engine setup, where all involved systems and changes in energy are accounted for explicitly. Let us begin with the total Hamiltonian

$$
\hat{H}_{\text {tot }}=\hat{H}_{\mathrm{Cold}}+\hat{H}_{\mathrm{Hot}}+\hat{H}_{\mathrm{M}}+\hat{H}_{\mathrm{W}},
$$




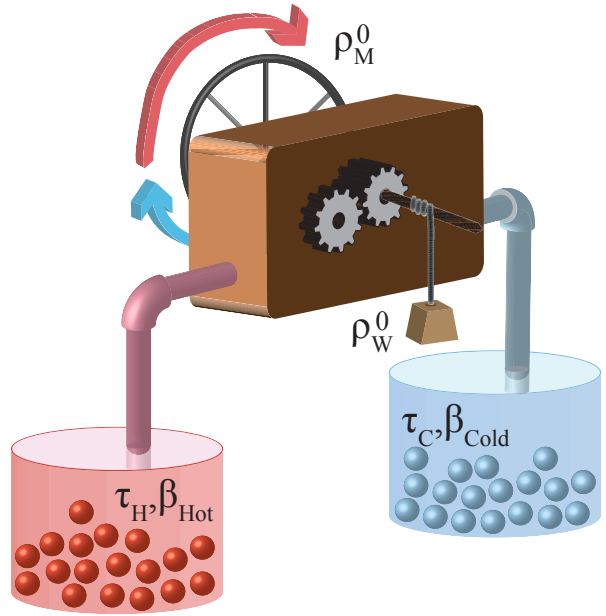

Figure 1: Illustration of a heat engine that contains four main components: two baths at different temperatures, a machine and a system we call a battery.

where the indices Hot, Cold, M, W represent a hot thermal bath (Hot), a cold thermal bath (Cold), a machine $(\mathrm{M})$, and a battery $(\mathrm{W})$ respectively. We adopt a resource theoretic approach, which allows all energy-preserving unitaries $U(t)$ on the global system, i.e. all unitaries which obey

$$
\left[U(t), \hat{H}_{\text {tot }}\right]=0 .
$$

Note that this global, time-independent Hamiltonian, is non-interacting; which is an indispensable feature of the resource theory framework [6, 5]. This approach allows us to mark a clear separation of the subsystems, and thus justifies the use of Gibbs states as thermal reservoirs. This is because for any given Hamiltonian, the Gibbs states uniquely satisfy complete passitivity [7, 42], which forbids the extraction of average energy via unitary operations on the state. Nevertheless, interactions of arbitrary strength between all the different systems are still allowed, under the only condition of Eq. (3). Indeed, $U(t)$ can be of the form

$$
U(t)=\mathrm{e}^{\mathrm{i} t\left(\hat{H}_{\mathrm{tot}}+\hat{I}_{\text {ColdHotMw }}\right)},
$$

with the norm of $\hat{I}_{\text {ColdHotMw }}$ being arbitrarily large, so long as $\left[\hat{I}_{\text {ColdHotMW }}, \hat{H}_{\text {tot }}\right]=0$ in order to preserve energy. In order to implement the unitary over the heat engine, one may use an auxiliary system. This is an aspect of the model which is common to all resource theory approaches to quantum thermodynamics. The optimal way to achieve this remains an open question. See [43] for partial results and Section V in [4] for a more in-depth discussion.

The initial state of our heat engine will be of the form

$$
\rho_{\text {ColdHotMW }}^{0}=\tau_{\text {Cold }}^{0} \otimes \tau_{\text {Hot }}^{0} \otimes \rho_{\mathrm{M}}^{0} \otimes \rho_{\mathrm{W}}^{0} .
$$

The state $\tau_{\text {Hot }}^{0}\left(\tau_{\text {Cold }}^{0}\right)$ is the initial thermal state at inverse temperature $\beta_{\text {Hot }}\left(\beta_{\text {Cold }}\right)$, corresponding to the hot (cold) bath Hamiltonians $\hat{H}_{\text {Hot }}, \hat{H}_{\text {Cold }}$, with $\beta_{\text {Cold }}>\beta_{\text {Hot }}$. More generally, given any Hamiltonian $\hat{H}$ and inverse temperature $\beta$, the thermal state is defined as $\tau=$ $\frac{1}{\operatorname{tr}\left(e^{-\beta \hat{H}}\right)} e^{-\beta \hat{H}}$. The initial machine $\left(\rho_{\mathrm{M}}^{0}, \hat{H}_{\mathrm{M}}\right)$ can be chosen arbitrarily, as long as its final state is preserved, and therefore the machine acts like a catalyst. Lastly, the initial battery state in our setup $\rho_{\mathrm{W}}^{0}$ is any energy eigenstate of the battery - see Section 2.2 for a further description of the battery model used in this manuscript.

Heat engines in practice tend to have their hot and cold thermal baths of different relative sizes. Power stations near the ocean are good examples of this. Here we allow our baths to play a similar role. The hot bath may be arbitrarily large, and acts like a reservoir, while the cold bath is of some fixed finite size. A reversal of the hot and cold bath sizes would be possible within our framework but unnecessary to reach our conclusions.

One cycle of the heat engine process produces a final reduced state $^{3}$

$$
\rho_{\text {ColdMW }}^{1}=\operatorname{tr}_{\text {Hot }}\left[U(t) \rho_{\text {ColdHotMW }}^{0} U(t)^{\dagger}\right],
$$

where the machine should be preserved, i.e. $\rho_{\mathrm{M}}^{1}=$ $\rho_{\mathrm{M}}^{0}$, and $\rho_{\text {Cold }}^{1}, \rho_{\mathrm{W}}^{1}$ are the final local states of the cold bath and battery. Note that we allow for arbitrary correlations to exist in the final state $\rho_{\text {ColdMW }}^{1}$, as long as the reduced state of the machine is preserved. Our central quantity of interest is the maximum efficiency of work extraction; and whether it can be as large as the Carnot efficiency. One may argue that allowing for arbitrary final correlations (quantum or classical) is not physically well motivated; since the correlations might degrade the functionality of the machine and potentially reduce the efficiency of the heat engine in subsequent cycles. Fortunately,

${ }^{3}$ For any bipartite state $\rho_{\mathrm{AB}}$, we use the notation of reduced states $\rho_{\mathrm{A}}:=\operatorname{tr}_{\mathrm{B}}\left(\rho_{\mathrm{AB}}\right), \rho_{\mathrm{B}}:=\operatorname{tr}_{\mathrm{A}}\left(\rho_{\mathrm{BA}}\right)$. 
this debate can be avoided, since we also show that if the heat engine setup is such that Carnot efficiency is achievable, then the final state of the heat engine must be of product form:

$$
\rho_{\text {ColdMW }}^{1}=\rho_{\text {Cold }}^{1} \otimes \rho_{\mathrm{M}}^{1} \otimes \rho_{\mathrm{W}}^{1} .
$$

The fact that correlations in the final state never allow, in all cases, for a larger amount of work nor an increased efficiency is proven in Section F.1 of the Supplementary Material. However, one can intuitively see why it is so, from the fact that the non-equilibrium free energy [44, 45, 46] is superadditive. More precisely, for any system of state $\rho$ in thermal contact with a bath at inverse temperature $\beta$, consider the non-equilibrium free energy $^{4}$

$$
F(\rho):=\operatorname{tr}(\hat{H} \rho)-\beta^{-1} S(\rho),
$$

where $S(\rho)=-\operatorname{tr}(\rho \ln \rho)$, is the von Neumann entropy. For our setup, super-additivity is then the statement that for any bipartite state $\rho_{A B}$, $F\left(\rho_{A B}\right) \geq F\left(\rho_{A}\right)+F\left(\rho_{B}\right)$, where equality is achieved if and only if $A$ and $B$ are uncorrelated. This implies that creating a final state with correlations is at least as hard as creating one without correlations, and thus allowing the final components of the heat engine to become correlated, cannot help one to achieve the Carnot efficiency; which may be achieved only in the limit when $F(\cdot)$ is invariant under one cycle of the heat engine (i.e. the limit in which the heat engine is macroscopically reversible $)^{5}$. As such, since a heat engine process needs to satisfy the non-increase of the free energy, it turns out that without loss of generality we can assume $\rho_{\text {ColdMW }}^{1}$ to be of the form given in Eq. (7) when investigating the achievability of Carnot efficiency in our setup. We therefore base the rest of our analysis in the main text on Eq. (7).

Since $\left(\tau_{\text {Hot }}^{0}, \hat{H}_{\mathrm{Hot}}\right)$ and $\left(\rho_{\mathrm{M}}^{0}, \hat{H}_{\mathrm{M}}\right)$ can be arbitrarily chosen and since Eq. (7) is assumed, the

\footnotetext{
${ }^{4}$ For systems in thermodynamics equilibrium, this free energy is also known as the Helmholtz free energy. For simplicity, we will also refer to this quantity as standard free energy in the text.

${ }^{5}$ As a side remark for readers familiar with [47], the reason why correlating catalysts do not boost efficiency in our set-up, is because the dimension of the catalyst diverges in the limit approaching the Carnot efficiency. Furthermore, the techniques developed in this manuscript might potentially pave way to solving an open problem in [47], as pointed out by the authors.
}

setup now corresponds to the set of catalytic thermal operations [7] one can perform on the joint state ColdW. This implies that the cold bath is used as a resource state. By catalytic thermal operations that act on the cold bath, using the hot bath as a thermal reservoir, and the machine as a catalyst, one can possibly extract work and store it in the battery. In the next section, we see how work is defined and categorized according to initial and final states of the battery $\rho_{\mathrm{W}}^{0}$ and $\rho_{\mathrm{W}}^{1}$. As for now, to summarize, the following assumptions are made in our heat engine setup:

1. The initial global state is a product state between all the systems, as shown in Eq. (5).

2. Cyclicity of the machine, i.e. system $M$ undergoes a cyclic process: $\rho_{\mathrm{M}}^{0}=\rho_{\mathrm{M}}^{1}$.

3. The heat engine as a whole is isolated from and does not interact with the world, i.e. $\left[U(t), \hat{H}_{\text {tot }}\right]=0$. This assumption ensures that all possible resources in a work extraction process has been accounted for.

4. The Hilbert space associated with $\rho_{\text {ColdHotMW }}^{0}$ is finite dimensional but can be arbitrarily large.

\subsection{Work in the nanoregime}

The definition of work when dealing with nanoscopic quantum systems has seen much attention lately [6, 7, 8, 9, 11, 12]. Performing work is always understood as changing the energy of a system, which in this manuscript is called battery. In the macroregime, one often pictures raising a weight on a string. In the nanoregime, this corresponds to changing the energy of a quantum system by pumping it to an excited state (see Fig. 2). In particular, a minimalistic battery model can be demonstrated as a two-level system [7]. Performing work corresponds to bringing the state from its ground state to the excited state, where the energy gap is fine-tuned to the amount of work $W_{\text {ext }}$ to be done.

While an arbitrary energy spacing is difficult to realize in a two-level system, it can be done by picking two levels with the desired spacing from a quasi-continuum battery: this battery comprises of a large but finite number of discrete levels which form a quasi-continuum. Such a battery closely resembles the classical notion of a "weight 
attached to a string" as considered in [37]. The battery can be charged by bringing it from a particular state (e.g. the ground state) to any of the higher energy levels. In this paper, we adopt the use of such a quasi-continuum battery model. This battery $W$ has a Hamiltonian (written in its diagonal form)

$$
\hat{H}_{\mathrm{W}}:=\sum_{i=1}^{n_{\mathrm{W}}} E_{i}^{\mathrm{W}}\left|E_{i}\right\rangle\left\langle\left. E_{i}\right|_{\mathrm{W}},\right.
$$

where $\left\{E_{i}^{\mathrm{W}}\right\}_{i=1}^{n_{\mathrm{W}}}$ is a set which can be arbitrarily large, but of fixed cardinality; while its elements $E_{i}^{\mathrm{W}} \in \mathbb{R}$ may or may not be uniformly bounded.
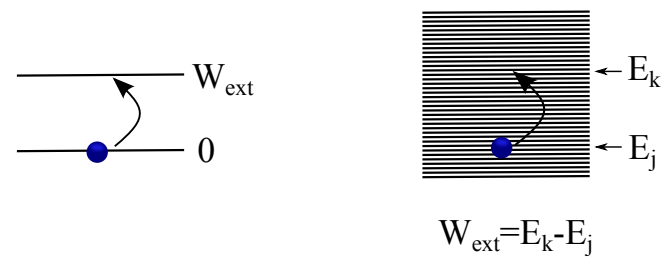

Figure 2: The battery models a work-storage component of a heat engine. In our setup, an adaptive quasicontinuum of energy levels is used.

One aspect of extracting work $W$ is to bring the battery's initial state $\rho_{\mathrm{W}}^{0}$ to some final state $\rho_{\mathrm{W}}^{1}$ such that $W=\operatorname{tr}\left(\rho_{\mathrm{W}}^{1} \hat{H}_{\mathrm{W}}\right)-\operatorname{tr}\left(\rho_{\mathrm{W}}^{0} \hat{H}_{\mathrm{W}}\right)>0$. However, a change in energy alone, does not yet correspond to performing work. It is implicit in our macroscopic understanding of work that the energy transfer takes place in an ordered form. When lifting a weight, we know its final position and can exploit this precise knowledge to transfer all the work onto a third system without - in principle - losing any energy in the process.

In the quantum regime, such knowledge corresponds to $\rho_{\mathrm{W}}^{1}$ being a pure state. When $\rho_{\mathrm{W}}^{1}$ is diagonal in the energy eigenbasis of $\hat{H}_{\mathrm{W}}$, then $\rho_{\mathrm{W}}^{1}$ is an energy eigenstate. We can thus understand work as an energy transfer about which we have perfect information, while heat, in contrast, is an energy transfer about which we hold essentially no information, other than average energy increase. Clearly, there is also an intermediary regime in which we transfer energy, while having some, but imperfect information.

To illustrate this, consider the quasi-continuum battery described above, and starting out from an arbitrary initial energy eigenstate $\rho_{\mathrm{W}}^{0}=$ $\left|E_{j}\right\rangle\left\langle\left. E_{j}\right|_{\mathrm{W}}\right.$. Ideally, we want to extract work and store it in the battery, by inducing a transition to another energy eigenstate $\left|E_{k}\right\rangle\left\langle\left. E_{k}\right|_{\mathrm{W}}\right.$, where $E_{k}^{\mathrm{W}}>E_{j}^{\mathrm{W}}$. Let $\varepsilon$ denote the failure probability of our doing so, and

$$
W_{\text {ext }}=E_{k}^{\mathrm{W}}-E_{j}^{\mathrm{W}}>0
$$

the work extracted. In the case where $W_{\text {ext }}$ is a value such that the transition $\rho_{\text {ColdW }}^{0} \rightarrow \rho_{\text {ColdW }}^{1}$ is possible via catalytic thermal operations, it corresponds to extracting work. We first define the case in which we always succeed:

Definition 1. The work extracted $W_{\text {ext }}$, is called perfect work when $\rho_{\mathrm{W}}^{1}=\left|E_{k}\right\rangle\left\langle\left. E_{k}\right|_{\mathrm{W}}\right.$, in other words, $\varepsilon=0$.

In general however, there is a non-zero failure probability of work extraction. This causes us to lose some information about the battery state: rather than the final state of the battery being $\left|E_{k}\right\rangle\left\langle\left. E_{k}\right|_{\mathrm{W}}\right.$, it could be any state which is a distance $\varepsilon$ away, namely

$$
d\left(\rho_{\mathrm{W}}^{1},\left|E_{k}\right\rangle\left\langle\left. E_{k}\right|_{\mathrm{W}}\right)=\varepsilon,\right.
$$

where we use $d(\rho, \sigma)$ to denote the trace distance of two states $\rho$ and $\sigma$ (see Eq. (44) for a definition). The usage of trace distance here is motivated by its strong operational meaning: the smaller the trace distance between two states, the harder it is to distinguish them using any quantum measurements. We allow for any value of

$$
\varepsilon \in[0, l], \text { for any fixed } l<1 \text {, }
$$

and the smaller $\varepsilon$ is, the closer we are to the situation of perfect work ${ }^{6}$. Note that any energy incoherent state $\rho_{W}^{1}$ such that $d\left(\rho_{W}^{1},\left|E_{k}\right\rangle\left\langle E_{k}\right|\right)=\varepsilon$ can be written in the form

$$
\rho_{W}^{1}=(1-\varepsilon)\left|E_{k}\right\rangle\left\langle E_{k}\right|+\varepsilon \rho_{\text {junk }},
$$

where $\rho_{\text {junk }}$ is any density matrix of the battery that does not have support on $\left|E_{k}\right\rangle\left\langle E_{k}\right|$. The parameter $W_{\text {ext }}>0$ is defined as an energy difference in Eq. (10). Our goal is to maximize the achievable value of $W_{\text {ext }}$, while allowing for all final battery states of the form of Eq. (13), such

${ }^{6}$ In Eq. (12), the constraint of a fixed $l<1$, is simply to rule out the physically irrelevant limit $\varepsilon \rightarrow 1$, where the final state has no overlap at all with $\left|E_{\mathrm{W}}^{k}\right\rangle\left\langle E_{\mathrm{W}}^{k}\right|$. We could choose $l=0.999$ for example. 
that there exists a catalytic thermal operation enabling the transition $\rho_{\text {ColdW }}^{0} \rightarrow \rho_{\text {ColdW }}^{1}$.

In this manuscript, we propose a characterization of the quality of extracted energy by the entropy difference

$$
\Delta S:=S\left(\rho_{\mathrm{W}}^{1}\right)-S\left(\rho_{\mathrm{W}}^{0}\right),
$$

where $S(\rho)=-\operatorname{tr}(\rho \ln \rho)$, is the von Neumann entropy. For perfect work, $\Delta S=0$. Another type of example one sometimes comes across, is when $\rho_{\mathrm{W}}^{1}$ becomes a thermal state $[29,31]$. In such cases, although the average energy of the battery may still increase, the corresponding entropy increase is maximal [48]. Moreover, when $\Delta S>0$, what is relevant is not its value as an absolute, but relative to the energy $W_{\text {ext }}$ that is extracted. We are thus interested in the quantity $\Delta S / W_{\text {ext }}$, in particular the limit $\Delta S / W_{\text {ext }} \rightarrow 0$ ${ }^{7}$, which motivates the following definition:

Definition 2. (Near perfect work) We say that a partially ordered set of heat engine protocols characterized by the extracted amount of work and and corresponding failure parameters $\mathcal{S}^{\text {H.E. }}=$ $\left\{\left(W_{\mathrm{ext}}, \varepsilon\right)\right\}$, leads to near perfect work extraction if

1) For all values of $\varepsilon, 0<\varepsilon \leq l$, for some fixed $l<1$ and

2) For all $1>p>0$, there exists a non-trivial subset of protocols $\mathcal{S}_{p}^{\text {H.E. }} \subset \mathcal{S}^{\text {H.E. }}$ such that when $\left(W_{\text {ext }}, \varepsilon\right) \in \mathcal{S}_{p}$, then

$$
\frac{\Delta S}{W_{\text {ext }}}<p
$$

We shall see in Section 3 that the maximum efficiency of a heat engine cycle can only be achieved in the limit where $W_{\text {ext }} \rightarrow 0$. In such cases, condition 2) in Def. 2 also corresponds to the limit $\Delta S / W_{\text {ext }} \rightarrow 0$. In Section A of the Supplementary Material, we show that the restriction of near perfect work can be re-cast in terms of an equivalent condition involving the probability of failure $\varepsilon$, namely: the two conditions of Definition 2 above are satisfied iff

$$
\lim _{\varepsilon \rightarrow 0^{+}} \frac{\Delta S}{W_{\text {ext }}}=0 .
$$

\footnotetext{
${ }^{7}$ Note that $\Delta S$ and $W_{\text {ext }}$ have different units. If one prefers to work with a unitless measure, one can instead work with $c \cdot \Delta S / W_{\text {ext }}$ for any constant $c$ with units of inverse temperature w.l.o.g., since the limit $c \cdot \Delta S / W_{\text {ext }} \rightarrow$ 0 holds iff $\Delta S / W_{\text {ext }} \rightarrow 0$ holds.
}

Perfect and near perfect work are the two types of energy investigated in this paper, due to their strong operational significance in capturing both the essence of energy increase and knowledge about the final battery state. Other types of energy increase, which we refer to as imperfect work, are studied in a separate paper, due to the large qualitative differences in the extracted energy [49]. For example, it is worth noting that for both perfect and near perfect work, one may recover Carnot's results about the efficiency of heat engines by invoking the non-increase of free energy (see Section 3.3); this is not the case for imperfect work, where one can surpass the Carnot efficiency, since $\Delta S$ is non-negligible compared to $W_{\text {ext }}$; thus heat contributions are not separated from the extracted energy. From another perspective, this means that imperfect work is a more debatable way of quantifying energy as work, nevertheless, this is addressed in [49] due to the extensive literature on quantum thermodynamics that uses mean energy increase as a quantifier of work.

\subsection{Definition of efficiency and maximum effi- ciency}

The efficiency of a heat engine is defined as

$$
\eta:=\frac{W_{\text {ext }}}{\Delta H}
$$

where $\Delta H$ is the amount of heat drawn from the hot bath, namely $\Delta H=\operatorname{tr}\left(\hat{H}_{\text {Hot }} \rho_{\text {Hot }}^{0}\right)-$ $\operatorname{tr}\left(\hat{H}_{\mathrm{Hot}} \rho_{\mathrm{Hot}}^{1}\right)$. Since the machine's final and initial states are the same after one cycle, and the initial state of the cold bath is fixed; due to total mean energy conservation, $\Delta H$ can be expressed solely as a function of $\rho_{\text {Cold }}^{1}$ and $W_{\text {ext }}$. From Eq. (17), we can define the maximum achievable efficiency in the nanoregime $\eta^{\text {nano }}$ as a function of the final state of the cold bath $\rho_{\text {Cold }}^{1}$. More precisely,

$$
\begin{aligned}
& \eta^{\text {nano }}\left(\rho_{\text {Cold }}^{1}\right):=\sup _{W_{\text {ext }}>0} \eta\left(\rho_{\text {Cold }}^{1}\right) \quad \text { subject to } \\
& F_{\alpha}\left(\rho_{\mathrm{W}}^{0} \otimes \tau_{\text {Cold }}^{0}\right) \geq F_{\alpha}\left(\rho_{\mathrm{W}}^{1} \otimes \rho_{\text {Cold }}^{1}\right) \quad \forall \alpha \geq 0,
\end{aligned}
$$

where $F_{\alpha}$ are the generalized free energies (see Eq. (30) in Section 4 for definition). Note that by fulfilling Eq. (19), we are already maximizing over all possible heat engine cycles for a given value of 
$W_{\text {ext }}$. Eq. (18) further maximizes efficiency over achievable values of $W_{\text {ext }}$. Recall that $\beta_{\text {Cold }}, \beta_{\text {Hot }}$ are fixed, and $\rho_{\mathrm{W}}^{0}$ is an energy eigenstate. In the resource theoretic framework for thermodynamics, the generalized free energies provide conditions for the possibility of catalytic thermal operations between the initial and final states to take place. In other words, Eq. (19) is an application of the so-called generalized second laws of quantum thermodynamics, to our heat engine setup; as shown in detail in Section 4. They can be seen as a generalization of the second law of thermodynamics (see Section 3.3), in single-shot quantum thermodynamics. From Eq. (30), we see that the constraint of Eq. (19) is equivalent to $D_{\alpha}\left(\rho_{\mathrm{W}}^{0} \otimes \tau_{\text {Cold }}^{0} \| \tau_{\text {ColdW }}^{h}\right) \geq D_{\alpha}\left(\rho_{\mathrm{W}}^{1} \otimes \rho_{\text {Cold }}^{1} \| \tau_{\text {ColdW }}^{h}\right)$ for all $\alpha \geq 0$, where $\tau_{\text {ColdW }}^{h}$ is the Gibbs state of the cold bath and battery at inverse temperature $\beta_{h}$, and $D_{\alpha}$ are called $\alpha$-Rényi divergences. The family of $\alpha$-Rényi divergences (which the max relative entropy discussed later is a member of) have been a powerful tool in single-shot quantum information theory, when it comes to tasks such as randomness extraction [50, 51, 52], source coding $[50,53]$, or hypothesis testing [54, 55] for finite number of trials.

With Eqns. (18) and (19) at hand, one can now define the maximum efficiency across all final states in the cold bath Hilbert space $\mathcal{S}\left(\mathcal{H}_{\text {Cold }}\right)$, when demanding near perfect work. Specifically,

$$
\eta_{\max }=\sup _{\rho_{\text {Cold }}^{1} \in \mathcal{S}\left(\mathcal{H}_{\text {Cold }}\right)} \eta^{\text {nano }}\left(\rho_{\text {Cold }}^{1}\right)
$$

where the supremum is also over all partially ordered heat engine protocols corresponding to near perfect work. In our analysis, a particular notion of efficiency emerges as the quantity of interest, which we refer to as the quasi-static limit. This corresponds to the maximum efficiency when the final state of the cold bath is thermal and its temperature only increases by an infinitesimal amount, namely

$$
\eta_{\max }^{\text {stat }}=\lim _{g \rightarrow 0^{+}} \eta^{\text {nano }}(\tau(g))
$$

where $\tau(g)$ is the Gibbs state on $\mathcal{S}\left(\mathcal{H}_{\text {Cold }}\right)$ at inverse temperature $\beta_{f}=\beta_{\text {Cold }}-g$. The reason why this limit emerges as a relevant scenario is as follows: first of all, we show that $\eta^{\text {nano }}\left(\rho_{\text {Cold }}^{1}\right)$ depends on two quantities, namely the average energy change in the cold bath (denoted as $\Delta C$ throughout this manuscript), and the extractable work $W_{\text {ext }}$. Given any fixed amount of $\Delta C>0$, maximizing the efficiency over $\rho_{\text {Cold }}^{1}$ corresponds to further maximizing $W_{\text {ext }}$ according to the $F_{1}$ constraint in Eq. (19). Moreover, $F_{1}$ is precisely the non-equilibrium free energy in Eq. (8). We show that this maximum $W_{\text {ext }}$ occurs precisely when $\rho_{\text {Cold }}^{1}=\tau(g)$ for the particular value of $g$ that corresponds to the fixed $\Delta C$. Furthermore, we also prove that the efficiency is monotonically decreasing with this parameter $g$, which means that the maximum efficiency occurs at the quasi-static limit described in Eq. (20). Indeed, if one evaluates the efficiency taking the quasistatic limit when assuming that only the condition on $F_{1}$ needs to be satisfied, one obtains the Carnot efficiency $\eta_{C}$ in the limit $g \rightarrow 0^{+}$. Since this is a necessary condition for the possibility of a heat engine process, we will thus frequently work in the quasi-static limit in the rest of this manuscript.

\section{Main results}

\subsection{No perfect work}

Before establishing our main result, we first show that in the nanoscopic regime, no heat engine can output perfect work (Def. 1). That is, the efficiency of any such heat engine,

$$
\sup _{\rho_{\text {Cold }}^{1} \in \mathcal{S}\left(\mathcal{H}_{\text {Cold }}\right)} \eta^{\text {nano }}\left(\rho_{\text {Cold }}^{1}\right)=0 .
$$

In other words, there exists no global energy preserving unitary $U(t)$ obeying Eq. (3) for which $W_{\text {ext }}>0$ can be achieved. The proof of this statement can be found in Section E.1 of the Supplementary Material. In fact, it is interesting to note that the impossibility of drawing perfect work is a direct consequence of needing to satisfy one instance of the generalized second laws, in particular $F_{\alpha}(\rho)$ in Eq. (19) when $\alpha=0$.

While this might appear puzzling at first glance, it has a very nice analog in information theory; namely zero-error data compression. The scenario is as follows: suppose one desires to send a message across a particular channel. Depending on the redundancy of your data, you might not need to send the full file over: you can send a compressed version of the data, that only sends a fraction of symbols. But if your data is distributed over symbols with respect to a probability distribution of full rank, then theoretically 
you cannot perform compression with precisely zero error - compression may be possible, however, if very small errors are allowed.

\subsection{Obtainable efficiency}

Clearly, however, it is unreasonable to say that no heat engine could work at all in the quantum nanoregime, prompting the question how this might be possible. We show that for any $\varepsilon>0$, there exists a heat engine such that $W_{\text {ext }}>0$ can be achieved. Therefore, a heat engine is possible if we ask only for near perfect work. Interestingly, even in the macroscopic regime, we can envision a heat engine that only extracts work with probability $1-\varepsilon$, but over many cycles of the engine we do not notice this feature when looking at the average work gained in each run.

To study the efficiency in the nanoscale regime we make crucial use of the second laws of quantum thermodynamics [7]. It is apparent from these laws that we might only discover further limitations to the efficiency than we see at the macroscopic scale. Indeed they do arise, as we find that the efficiency no longer depends on just the temperatures of the heat baths. Instead, the explicit structure of the cold bath Hamiltonian $\hat{H}_{\text {Cold }}$ becomes important (a similar argument can be made for the hot bath) - even when choosing the optimal machine.

We conduct the full analysis of the efficiency according to the second laws of thermodynamics considering a cold bath comprised of $n$ noninteracting two-level systems (qubits) each with its own energy gap $\bar{E}_{k}$,

$$
\hat{H}_{\mathrm{Cold}}=\sum_{k=1}^{n} \mathbb{1}^{\otimes(k-1)} \otimes \bar{E}_{k}\left|\bar{E}_{k}\right\rangle\left\langle\bar{E}_{k}\right| \otimes \mathbb{1}^{\otimes(n-k)}
$$

where $n$ can be arbitrarily large, but finite. Let us denote the spectral gap of the cold bath the energy gap between its ground state and first excited state - by $E_{\min }$. We can then define the quantity

$$
\Omega=\frac{E_{\min }\left(\beta_{\text {Cold }}-\beta_{\text {Hot }}\right)}{1+e^{-\beta_{\text {Cold }} E_{\min }}},
$$

Whenever $\Omega \leq 1$, consider all qubits on sites $k$ for which

$$
\frac{\bar{E}_{k}\left(\beta_{\text {Cold }}-\beta_{\text {Hot }}\right)}{1+e^{-\beta_{\text {Cold }} \bar{E}_{k}}} \leq 1,
$$

holds. Since $\Omega \leq 1$ in this case, there will be a non-trivial subset $\mathcal{C}$ of the cold bath qubits (at least one qubit) where Eq. (25) holds. We show that the quasi-static efficiency $\eta_{\max }^{\text {stat }}$ (for which the cold bath is taken over $\mathcal{C}$ ) is indeed the familiar Carnot efficiency, which can be expressed as

$$
\eta_{\max }=\eta_{\max }^{\text {stat }}=\left(1+\frac{\beta_{\text {Hot }}}{\beta_{\text {Cold }}-\beta_{\text {Hot }}}\right)^{-1} .
$$

Note that this is true for any $n$ number of qubits, in particular also when $n=1$, which remarkably tells us that even when the cold bath consists of only a single qubit, Carnot efficiency can still be achieved when $\Omega \leq 1$ is satisfied. Intuitively, although the constrained optimization of Eq. (18) looks complicated in general, nevertheless only the constraint imposed by the $\alpha=1$ second law in Eq. (19) is the most stringent one, and the optimal transition is when $F\left(\rho_{\text {ColdW }}^{0}\right)=F\left(\rho_{\text {ColdW }}^{1}\right)$. The other constraints in Eq. (19) are trivially satisfied with an inequality. Therefore, the second laws give effectively the same constraint as the usual second law.

However, when $\Omega>1$, we find a new nanoscale limitation. In this situation, the efficiency for near perfect work is only

$$
\eta_{\max }^{\text {stat }}=\left(1+\frac{\beta_{\text {Hot }}}{\beta_{\text {Cold }}-\beta_{\text {Hot }}} \Omega\right)^{-1}
$$

for a quasi-static heat engine. Furthermore, note that for the case of a single qubit, all energyincoherent states are thermal states with a particular temperature, and therefore the quasi-static limit is the only possible parametrization for the limit $\rho_{\text {Cold }}^{1} \rightarrow \tau_{\text {Cold }}^{0}$. This means that for a singlequbit cold bath, if $\Omega>1$ holds, then Eq. (27) gives the maximum achievable efficiency, which will be strictly less than $\eta_{C}$.

Eq. (27) marks a limitation at the nano/quantum scale. This limitation occurs because in the constrained optimization of Eq. (19), unlike when $\Omega \leq 1$, the $\alpha=\infty$ second law poses the strongest constraint (even stronger than the constraint of the non-equilibrium free energy), and therefore becomes solely relevant in dictating the state transition. In particular, the $\alpha=1$ second law can only be satisfied with a strict inequality when $\Omega>1$. The $\alpha$-Rényi divergence $D_{\infty}$, corresponding to the $\alpha=\infty$ second law in Eq. (19), is a well-known quantity in single-shot information theory called the max relative entropy, 


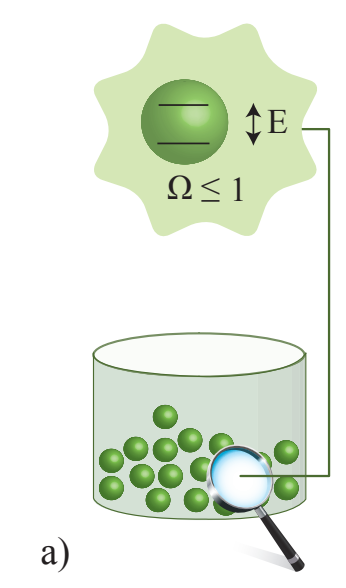

b)

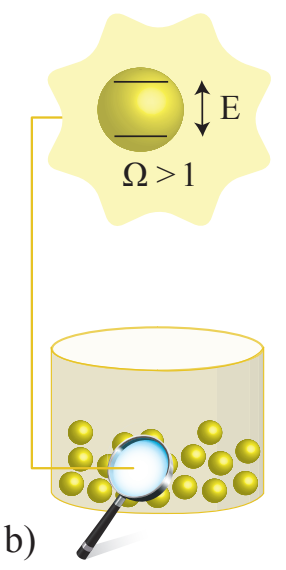

Figure 3: For fixed inverse temperatures $\beta_{\text {Cold }}$, $\beta_{\text {Hot }}$, the efficiency of a nanoscale heat engine depends on the structure of the cold bath. At the nano/quantum scale, Carnot's statement about the universality of heat engines does not hold. We find that the maximum efficiency of a heat engine, does not only depend on the inverse temperatures $\beta_{\text {Cold }}, \beta_{\text {Hot }}$ of the heat baths. In (a) the energy gaps are small enough to allow the heat engine to achieve Carnot efficiency, i.e., $\Omega \leq 1$. In (b) the efficiency of the heat engine is reduced below the Carnot efficiency because the energy gap of the qubits are above the critical value $\Omega>1$.

denoted by $D_{\max }[56,57]$. As such, in this regime, we find that instead of the non-equilibrium free energy, it is the max relative entropy that determines the efficiency of the heat engine cycle. The emergence of the max relative entropy as the deciding factor in obtainable efficiency is a signature of single-shot effects coming into play. In the quantum thermodynamics literature, it has also been shown that the max relative entropy dictates the minimum amount of input work required to create a state via catalytic thermal operations [6].

The restriction of near perfect work per cycle can now be further justified by examining how well the heat engine performs when the machine runs over many cycles: we find that if $\Omega \leq 1$, the heat engine can be run quasi-statically with an efficiency arbitrarily close to the Carnot efficiency while extracting any finite amount of work with an arbitrarily small entropy increase in the battery. This follows from repeatedly applying our single-shot results in the $\Omega \leq 1$ regime, as shown in Section E.3 of the Supplementary Material.

\subsection{Comparison to standard free energy results}

For any system in thermal contact with a bath at inverse temperature $\beta$, consider the nonequilibrium free energy defined in Eq. (8). In the macroregime, the usual second law states that the non-equilibrium free energy never increases,

$$
F\left(\rho_{0}\right) \geq F\left(\rho_{1}\right),
$$

when the system goes from a state $\rho_{0}$ to a state $\rho_{1}$. Note that this quantity, $F(\rho)$ is defined for arbitrary non-equilibrium states [58, 46], where $\beta$ is simply the inverse temperature of its surrounding bath. In the setting where one averages over infinitely many cycles, this quantity has been shown to correspond to the amount of work extractable from a generic, non-equilibrium state [46]. It also implies that $F(\rho)$ dictates the possibility of asymptotically transforming $n$ copies of $\rho_{0}$ into $\rho_{1}$, in the limit where $n \rightarrow \infty$.

In the single-shot quantum regime, however, Eq. (28) is but one of many conditions necessary for a state transformation. The generalized second laws [7] are a core result of quantum thermodynamics, that were derived based only on the foundations of quantum theory. Using these second laws, not only that many physical assumptions in classical thermodynamics can be avoided, but also one sees a more refined structure of the second law expressed in the context of nanoscale quantum systems. The limitations we observe on the efficiency are a consequence of having such generalized second laws.

From another perspective, the fact that more laws appear in this regime can intuitively be understood as being analogous to the fact that when performing a probabilistic experiment only a handful of times, not just the average, but other moments of a distribution become relevant. Indeed, all second laws converge to the standard second law in the limit of infinitely many particles [7], illustrating why we are traditionally accustomed to only this second law. The standard second law also emerges in some regimes of inexact catalysis [7], however, this corresponds to a degradation of the machine in each cycle, which would need one to use work to repair. Another example of a regime where the second law emerges, is when the catalysts are allowed to become correlated among themselves or with the rest of the machine $[59,47]$; however, in the limiting case in which only the second law is rele- 
vant, the dimension of the catalysts diverge. Furthermore, correlations between catalysts and the other states may require some additional work to destroy, hence making it less clear how to faithfully characterise the net extracted work.

It is illustrative to analyze our problem when we apply just the standard second law in Eq. (28) to derive bounds on efficiency, which is indeed a matter of textbook thermodynamics [60]. However, we here apply the law precisely to the heat engine model as given in Section 2, in which all energy flows are accounted for and (near) perfect work is performed. One might wonder whether the limitations we observe are just due to either a limited model, or our demand for near perfect instead of average work, and might thus also arise even when invoking only the standard second law. That is, are these newfound limitations really a consequence of the need to obey a wider family of second laws, or would the standard free energy predict the same things when energy is quantized, and quantum correlations are possible?

We show independently of whether we consider perfect or near perfect work, that according to the standard free energy in Eq. (28), the maximum achievable efficiency is given by the Carnot efficiency. Furthermore, we recover Carnot's statement that the Carnot efficiency can be achieved for any cold bath (i.e. for a cold bath with any finite dimensional pure point spectrum). We also see that for this case, the Carnot efficiency can always be achieved for quasi-static heat engines. These results can be proven without invoking any additional assumptions than those laid out in Section 2. In contrast, usual proofs of the second law require assumptions such as reversibility or that the system is in thermodynamic equilibrium at all times. Therefore, with our setup we recover exactly what Carnot predicted, namely that the maximum efficiency of heat engines only depends on the temperatures of the hot and cold bath. This rules out that our inability to achieve what Carnot predicted according to the macroscopic laws of thermodynamics is not merely the consequence of an overly stringent heat engine model, or definition of work.

Finally, it is important to note that there have been several recent works $[10,28,37,29,31,18$, $61,62]$ on analyzing the efficiencies of small quantum heat engines, and had achieved Carnot efficiency. In [37], a protocol was even constructed to achieve the Carnot efficiency for any system Hamiltonian and any arbitrary quantum state albeit considering operations which only preserve total energy on average. However, more common to all these approaches is that they consider an average notion of work, without directly accounting for a contribution from disordered energy (heat). Instead, one aims keeps the entropy of the battery low [37], or bound the higher moments of the energy distribution [29]. However, these only limit contributions from heat, but do not fully prevent them. Our notion of (near) perfect work now makes this aspect of macroscopic work explicit in the nanoregime, which has not been studied in the previous work. It is important to note that our work does not contradict previous results such as that of [37]. For example, the analysis of [37] shows that in each step of their protocol to achieve Carnot efficiency, the amount of energy change scales the same as the amount of entropy change, which does not correspond to perfect or near perfect work. Needless to say, imperfect work with some contribution of heat can also be useful in certain scenarios. Yet, it does not quite constitute work if we cannot explicitly single out a contribution from heat. One could construct a machine which extracts some amount of energy, with some non-negligible amount of information. It is proven in this case that Carnot efficiency can even be exceeded [49]. This should not come as a surprise, because we are no longer asking for work - energy transfer about which we have (near) perfect information.

\section{Proof Overview}

To quantify the amount of extractable work, we apply the generalized second laws derived in [7]. The initial cold bath $\rho_{\text {Cold }}^{0}$ is thermal, and therefore diagonal in the energy eigenbasis, while the initial battery state $\rho_{\mathrm{W}}^{0}$ is also a pure energy eigenstate (see Fig. 2). Since the unitary $U(t)$ is energy conserving, it will never increase coherences between global energy eigenstates [7]. We can therefore conclude that $\rho_{\text {ColdW }}^{1}$ is also diagonal in the energy eigenbasis. We can thus invoke the necessary and sufficient conditions for a transformation to be possible [7]. Specifically, $\rho_{\text {Cold }}^{0} \otimes \rho_{\mathrm{W}}^{0} \rightarrow \rho_{\text {ColdW }}^{1}$ iff $\forall \alpha \geq 0$,

$$
F_{\alpha}\left(\rho_{\text {Cold }}^{0} \otimes \rho_{\mathrm{W}}^{0}, \tau_{\text {ColdW }}^{h}\right) \geq F_{\alpha}\left(\rho_{\text {ColdW }}^{1}, \tau_{\text {ColdW }}^{h}\right),
$$


where $\tau_{\text {ColdW }}^{h}$ is the thermal state of the joint system (cold bath and battery) at temperature $T_{\text {Hot }}$. The generalized free energy $F_{\alpha}$ is defined as

$$
F_{\alpha}(\rho, \tau):=\frac{1}{\beta_{\mathrm{Hot}}}\left[D_{\alpha}(\rho \| \tau)-\ln Z_{\mathrm{Hot}}\right],
$$

where $D_{\alpha}(\rho \| \tau)$ are known as $\alpha$-Rényi divergences. For states $\rho, \tau$ which are diagonal in the same eigenbasis, the Rényi divergences can be simplified to

$$
D_{\alpha}(\rho \| \tau)=\frac{1}{\alpha-1} \ln \sum_{i} p_{i}^{\alpha} q_{i}^{1-\alpha}
$$

where $p_{i}, q_{i}$ are the eigenvalues of $\rho$ and $\tau$ respectively. The case $\alpha=1$ is defined by continuity in $\alpha$. Taking the limit $\alpha \rightarrow 1$ for Eq. (30), one recovers the non-equilibrium free energy, $F(\rho)=$ $\langle\hat{H}\rangle_{\rho}-\beta_{\mathrm{Hot}}^{-1} S(\rho)$. Using the second laws [7] is a powerful tool, since when searching for the optimum efficiency, we do not have to optimize explicitly over the possible machines $\left(\rho_{\mathrm{M}}, \hat{H}_{\mathrm{M}}\right)$, the form of the hot bath $\hat{H}_{\mathrm{Hot}}$, or the energy conserving unitary $U(t)$. Whenever Eq. (29) is satisfied, then we are guaranteed a suitable choice exists and hence we can focus solely on the possible final states $\rho_{\text {ColdW }}^{1}$.

Since we know that $\rho_{\text {ColdW }}^{1}$ is diagonal in the energy eigenbasis, the correlations between cold bath and battery can only be classical (w.r.t. energy eigenbasis). However, even such correlations cannot improve the efficiency: we show in the Supplementary Material that we may take the output state to have the form $\rho_{\text {ColdW }}^{1}=\rho_{\text {Cold }}^{1} \otimes$ $\rho_{\mathrm{W}}^{1}$ in order to achieve the maximum efficiency. According to Fig. 2, consider $\rho_{\mathrm{W}}^{0}=\left|E_{\mathrm{W}}^{j}\right\rangle\left\langle E_{\mathrm{W}}^{j}\right|$ and the final state

$$
\rho_{\mathrm{W}}^{1}=(1-\varepsilon)\left|E_{\mathrm{W}}^{k}\right\rangle\left\langle E_{\mathrm{W}}^{k}|+\varepsilon| E_{\mathrm{W}}^{j}\right\rangle\left\langle E_{\mathrm{W}}^{j}\right| .
$$

Although this is a particularly simple case of $\rho_{\mathrm{W}}^{1}$, we can show that it is actually sufficient for our analysis, i.e. allowing a more general battery state does not change the maximum efficiency. We do this by analyzing the generalized free energy $F_{\alpha}$ in the limit of $\alpha \rightarrow \infty$, and show that any other final battery state achieves at most the same maximum efficiency given by Eq. (32) (see Supplementary Material Section F.2). From the second laws Eq. (29), we may derive the maximum amount of extractable work, which is the largest value of $W_{\mathrm{ext}}=E_{\mathrm{W}}^{k}-E_{\mathrm{W}}^{j}$ such that the state transition $\rho_{\text {Cold }}^{0} \otimes \rho_{\mathrm{W}}^{0} \rightarrow \rho_{\text {Cold }}^{1} \otimes \rho_{\mathrm{W}}^{1}$ is possible. The form of $W_{\text {ext }}$ (derived in the Supplementary Material Section E.2.1) is

$$
\begin{aligned}
W_{\mathrm{ext}} & =\inf _{\alpha \geq 0} W_{\alpha} \\
W_{\alpha} & =\frac{1}{\beta_{\mathrm{Hot}}(\alpha-1)}\left[\ln \left(A-\varepsilon^{\alpha}\right)-\alpha \ln (1-\varepsilon)\right] \\
A & =\frac{\sum_{i} p_{i}^{\alpha} q_{i}^{1-\alpha}}{\sum_{i} p_{i}^{\prime \alpha} q_{i}^{1-\alpha}}
\end{aligned}
$$

where $p_{i}=\frac{e^{-\beta_{\mathrm{Cold}} E_{i}}}{Z_{\beta_{\mathrm{Cold}}}}, q_{i}=\frac{e^{-\beta_{\mathrm{Hot}} E_{i}}}{Z_{\beta_{\mathrm{Hot}}}}$ are probabilities of the thermal state of the cold bath at temperatures $\beta_{\text {Cold }}, \beta_{\text {Hot }}$ respectively, and $p_{i}^{\prime}$ are the probability amplitudes of state $\rho_{\text {Cold }}^{1}$ when written in the energy eigenbasis of $\hat{H}_{\text {Cold }}$. The quantity $W_{\text {ext }}$ is dependent on the initial and final cold bath $\rho_{\text {Cold }}^{0}, \rho_{\text {Cold }}^{1}$, the hot bath inverse temperature $\beta_{\text {Hot }}$, and the allowed failure probability $\varepsilon$. The difficulty of evaluating $W_{\text {ext }}$ comes from the infimum over $\alpha$, which is completely dependent on $\beta_{\text {Hot }}, \beta_{\text {Cold }}, \hat{H}_{\text {Cold }}$ and other parameters.

The efficiency $\eta$ defined in Eq. (17), however, is not determined by the maximum extractable work, but rather by an optimal tradeoff between $W_{\text {ext }}$ and the energy drawn from the hot bath, $\Delta$ Hot. Since $\hat{H}_{\text {ColdHotMw }}=\hat{H}_{\text {Cold }}+\hat{H}_{\text {Hot }}+\hat{H}_{\mathrm{M}}+$ $\hat{H}_{\mathrm{W}}$ is void of interaction terms, and since total energy is preserved, we can also write the change of energy in the hot bath, in terms of the energy change in the remaining systems,

$$
\Delta \text { Hot }=\Delta \text { Cold }+\Delta W .
$$

where $\Delta$ Cold $:=\operatorname{tr}\left[H_{\text {Cold }} \rho_{\text {Cold }}^{1}\right]-\operatorname{tr}\left[H_{\text {Cold }} \rho_{\text {Cold }}^{0}\right]$, and $\Delta W:=\operatorname{tr}\left(\hat{H}_{\mathrm{W}} \rho_{\mathrm{W}}^{1}\right)-\operatorname{tr}\left(\hat{H}_{\mathrm{W}} \rho_{\mathrm{W}}^{0}\right)$ are changes in average energy of the cold bath and battery. We thus see that the efficiency can be described solely in terms of the battery and the cold bath.

Macroscopic second law We first analyze the efficiency invoking only the standard second law, namely assuming that the free energy $(\alpha=1)$ fully dictates if a certain state transition is possible. The question is then: given an initial cold bath Hamiltonian $\hat{H}_{\text {Cold }}$, what is the maximum attainable efficiency considering all possible final states $\rho_{\text {Cold }}^{1}$ ? In both cases of perfect and near perfect work, we find that the efficiency is only maximized whenever $\rho_{\text {Cold }}^{1}$ is (A) a thermal state, and (B), when it is a thermal state, can only achieve the Carnot efficiency when the inverse temperature $\beta_{f}$ is arbitrarily close to $\beta_{\text {Cold }}$. 
We refer to this situation as a quasi-static heat engine. Moreover, we find that the Carnot efficiency can be achieved by any given $\hat{H}_{\text {Cold }}$. These results rigorously prove Carnot's findings when only the usual free energy is relevant.

Nanoscale second laws Here, when considering perfect work under the constraints of all generalized free energies coming into play, we are immediately faced with an obstacle: the constraint at $\alpha=0$ implies that $W_{\text {ext }}>0$ is not possible, whenever $\rho_{\text {Cold }}^{0}$ is of full rank. This is due to the discontinuity of $D_{0}$ in Eq. (31) w.r.t. the quantum state, and is similar to effects observed in information theory in lossy vs. lossless compression: no compression is possible if no error however small is allowed. However, when considering the limit $\Delta S / W_{\text {ext }} \rightarrow 0$, i.e. near perfect work, the $D_{0}$ constraint is satisfied automatically.

The results for the macroscopic second law form an upper bound for both the maximum extractable work and efficiency for nanoscale second laws, since the constraint of generalized free energy at $\alpha=1$ is one of the many constraints described by all $\alpha \geq 0$. We can thus show that the results from the standard second law have the following implication in the nanoregime: if we can achieve the Carnot efficiency, we can only do so when $\rho_{\text {Cold }}^{1} \rightarrow \tau_{\text {Cold }}^{0}$. We analyze the quasi-static regime. Furthermore, we specialize to the case where the cold bath consists of multiple identical two-level systems, each of which are described by a Hamiltonian with energy gap $E$.

Firstly, we identify characteristics that the failure parameter of work extraction $\varepsilon$ should have, such that near perfect work is extracted in the limit $\beta_{f} \rightarrow \beta_{\text {Cold }}$ (i.e. when (A) and (B) are satisfied). We then show two technical results:

1. The choice of $\varepsilon$ (as a function of $\beta_{f}$ ) simplifies the minimization problem in Eq. (33), by reducing the range of the variable $\alpha$ appearing in the optimization of $W_{\text {ext }}$. Under the consideration of near perfect work, $\varepsilon$ can be chosen such that the optimization of $\alpha$ is over $\alpha \geq \kappa$ for some $\kappa \in(0,1]$, instead of $\alpha \geq 0$. The larger $\kappa$ is for a chosen $\varepsilon$, the slower $\Delta S / W_{\text {ext }}$ converges to zero.

2. We analyze the following cases separately:

- For $\Omega \leq 1, \varepsilon$ can always be chosen so that Eq. (33) is obtained in the limit $\alpha \rightarrow 1$.
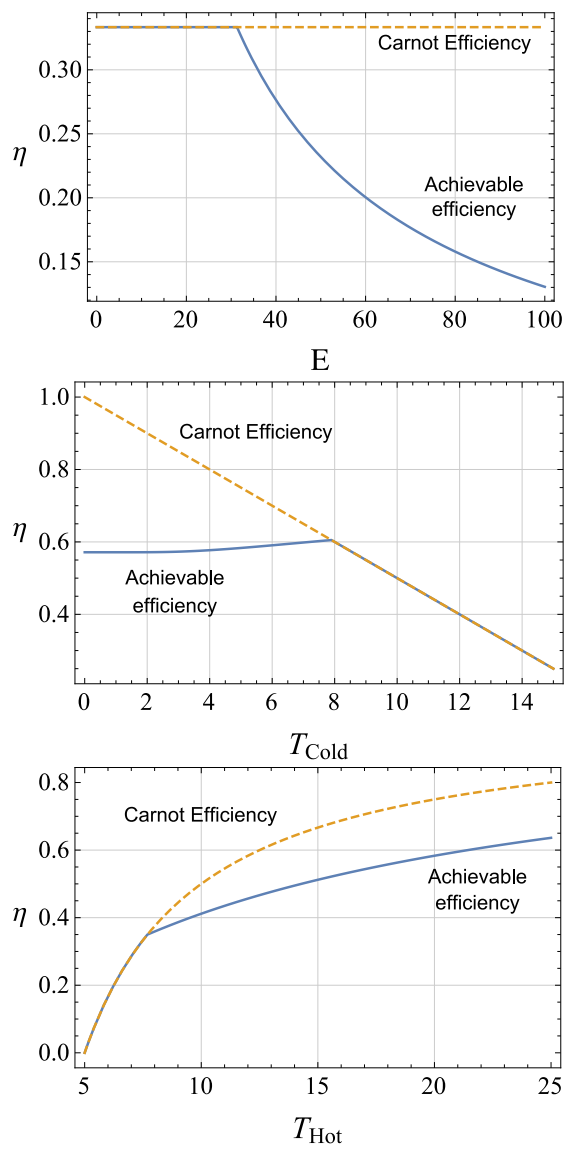

Figure 4: Comparison of the quasi-static nanoscale efficiency versus Carnot efficiency. Top: efficiency vs. the energy gap $E_{\min }$ of $\hat{H}_{\text {Cold }}$ (recall that $k_{\mathrm{B}}=1$ ). According to Eqs. (26), (27), for any $T_{\text {Cold }}<T_{\text {Hot }}$ one can achieve Carnot efficiency when $E_{\min }$ is sufficiently small. Otherwise, we find a reduced efficiency. This has been plotted for $T_{\text {Hot }}=15$ and $T_{\text {Cold }}=10$. Middle: efficiency vs. $T_{\text {Cold }}$, where $E_{\text {min }}=15$. For every $\hat{H}_{\text {Cold }}$, there exists a temperature regime ( $T_{\text {Cold }}$ vs. $\left.T_{\text {Hot }}\right)$ such that Carnot efficiency cannot be achieved. This happens as $T_{\text {Cold }}$ gets further from the temperature of the hot bath $T_{\text {Hot }}=20$. Bottom: efficiency vs. $T_{\mathrm{Hot}}$, where $E_{\min }=15$. Similarly, we see the inability of attaining Carnot efficiency, as $T_{\text {Hot }}$ increases relative to $T_{\text {Cold }}=5$. 
Evaluating the efficiency in the limit $\alpha \rightarrow 1$ corresponds to the Carnot efficiency.

- For $\Omega>1$, we show that for the best choice of $\varepsilon$, the infimum in Eq. (33) for $W_{\text {ext }}$ is obtained at $\alpha \rightarrow \infty$. Furthermore, $\Omega>1$ means that up to leading order terms (with regards to the quasi-static limit parameters), $W_{1}>W_{\infty}$ for $W_{\alpha}$ defined in Eq. (34). But we know that the quantity $W_{1}$ gives us Carnot efficiency. Therefore, the maximum efficiency is strictly less than the Carnot value.

\section{Discussions and Conclusion}

Our work establishes a fundamental result for the operation of nanoscale heat engines. We find all cold baths can be used in heat engines; and remarkably - that even when one of the heat baths is as small as a single qubit, as long as certain conditions of the bath Hamiltonian are met, Carnot efficiency can still be achieved in the quasi-static limit. However, Carnot efficiency is not necessarily always achieved for all baths; instead, achievability depends on the Hamiltonian structures of the baths. In the case where the cold bath consists of multiple qubits, we find that for all values of inverse temperatures $\beta_{\text {Cold }}$ and $\beta_{\text {Hot }}$ of the cold and hot baths, there exists an energy gap $E_{\min }\left(\beta_{\text {Cold }}, \beta_{\text {Hot }}\right)$ of the qubits forming the cold bath above, in which the optimal quasi-static efficiency is reduced below the Carnot efficiency. Viewed from another direction, for a fixed energy gap $E_{\min }\left(\beta_{\text {Cold }}, \beta_{\text {Hot }}\right)$, whether the Carnot efficiency can be achieved depends on the relation between $T_{\text {Hot }}$ and $T_{\text {Cold }}$ as illustrated in Fig. 4 . Loosely speaking, the Carnot efficiency can be achieved whenever the two temperatures are unequal but not too far apart. One might wonder why this restriction has not been observed before in the classical scenario. There, the energy spectrum of the bath is continuous or forms a quasicontinuum, and hence we always have access to the regime where Carnot efficiency is achievable.

Nanoscale heat engines are starting to be constructed experimentally [14, 20]. Not all of these nanoscale heat engines will be able to obtain the Carnot efficiency due to sub-optimality of the heat engines. Our results may influence future nanoscale machine designs, since engineers may wish to use thermal baths that have small energy gaps such that $\Omega$ is not too large, depending on the temperature difference between the two baths involved.

Our result is a consequence of the fact that the second law takes on a more complicated form in the nanoregime. It has been known for some time now that in addition to the standard second law, many other second laws become relevant and lead to additional restrictions. However, the implications of these restrictions on heat engines that operate in the quantum nanoregime have never been addressed in full until this paper. From a statistical perspective, small numbers require more refined descriptions than provided by averages, and as a result thermodynamics becomes more complicated when considering systems comprised of few particles. Similar effects can also be observed in information theory, where averaged quantities as given by the Shannon entropy need to be supplemented with refined quantities when we consider finitely many channel uses.

In the macroscopic regime, for completeness, we ruled out the possibility that the observed limitations on efficiency is a consequence of our demand for near perfect work, or the fact that we are using systems with discrete (sufficiently large spaced) spectra. This verification was achieved by showing that the Carnot efficiency can indeed always be attained (regardless to the size of an energy gap if present) when extracting near perfect work, when we are in such large systems that only the standard second law is relevant. One might wonder whether heat engines that do not operate by extracting an infinitesimal amount of work, or employing quantum coherences would allow us to achieve the Carnot efficiency independent of the structure of the cold bath. As we show in the Supplementary Material, both do not help.

It would furthermore be satisfying to derive the explicit form of a hot bath, and machine attaining Carnot - or our reduced Carnot - efficiency. One might wonder whether a non-trivial machine $\left(\rho_{\mathrm{M}}, \hat{H}_{\mathrm{M}}\right)$ is needed at all in this case. To illustrate the dependence on the bath, it was sufficient to consider a bath comprising solely of qubits. The tools proposed in the Supplementary Material can also be used to study other forms of bath structures, yet it is a non-trivial question to derive efficiencies for such cold baths.

Most interestingly, there is the extremely challenging question of deriving a statement that is 
analogous to the Carnot efficiency, but which makes explicit the trade-off between information and energy for all possible starting situations. In a heat engine, we obtain energy from two thermal baths about which we have minimal information. It is clear that the Carnot efficiency is thus a special case of a more general statement in which we start with two systems of a certain energy about which we may have some information, and we want to extract work by combining them. Indeed, the form that such a general statement should take is by itself a beautiful conceptual challenge, since what we understand as efficiency may not only be a matter of work obtained vs. energy wasted. Instead, we may want to take a loss of information about the initial states into account when formulating such a fully general efficiency.

\section{Acknowledgments}

We thank J. Oppenheim, M. Horodecki, R. Renner and A. Winter for useful comments and discussions. This research was funded by Singapore's MOE Tier 3A Grant MOE2012-T3-1-009, and STW, Netherlands. M.P.W. acknowledges funding from the Swiss National Science Foundation (Ambizione Fellowship PZ00P2_179914). N.H.Y.N. acknowledges support from the Alexander von Humboldt foundation.

\section{References}

[1] S. Carnot. Reflections on the Motive Power of Fire. 1824.

[2] R. Clausius. "Ueber verschiedene für die Anwendung bequeme Formen der Hauptgleichungen der mechanischen Wärmetheorie". In: Annalen der Physik 201.7 (1865), pp. 353-400. ISSN: 1521-3889.

[3] W. Thompson (Lord Kelvin). "On the Dynamical Theory of Heat, with numerical results deduced from Mr Joule's equivalent of a Thermal Unit, and M. Regnault's Observations on Steam". In: Transactions of the Royal Society of Edinburgh (1851). DOI: 10.1080/14786445208647064.
[4] N. Ng and M. P. Woods. "Resource Theory of Quantum Thermodynamics: Thermal Operations and Second Laws". In: Thermodynamics in the Quantum Regime: Fundamental Aspects and New Directions. Ed. by F. Binder et al. Springer International Publishing, 2018, pp. 625-650. ISBN: 978-3-319-99046-0. DOI: 10.1007/9783-319-99046-0_26.

[5] F. Brandão et al. "Resource theory of quantum states out of thermal equilibrium". In: Physical Review Letters 111.25 (2013), p. 250404. DOI: 10.1103/PhysRevLett.111.250404.

[6] M. Horodecki and J. Oppenheim. "Fundamental limitations for quantum and nano thermodynamics". In: Nature Communications 4.2059 (2013). DOI: 10.1038/ncomms3059.

[7] F. Brandão et al. "The second laws of quantum thermodynamics". In: Proceedings of the National Academy of Sciences 112.11 (2015), pp. 3275-3279. DOI: 10.1073/pnas.1411728112.

[8] J. Åberg. "Truly work-like work extraction via a single-shot analysis". In: Nature Communications 4 (2013). DOI: 10.1038/ncomms2712.

[9] O. Dahlsten et al. "Inadequacy of von Neumann entropy for characterizing extractable work". In: New Journal of Physics 13.5 (2011), p. 053015. DOI: 10.1088/1367-2630/13/5/053015.

[10] R. Uzdin, A. Levy, and R. Kosloff. "Quantum heat machines equivalence, work extraction beyond markovianity, and strong coupling via heat exchangers". In: Entropy 18.4 (2016), p. 124. DOI: 10.3390/e18040124.

[11] R. Gallego, J. Eisert, and H. Wilming. "Thermodynamic work from operational principles". In: New Journal of Physics 18.10 (2016), p. 103017. DOI: 10.1088/13672630/18/10/103017.

[12] J. Gemmer. and J. Anders. "From singleshot towards general work extraction in a quantum thermodynamic framework". In: New Journal of Physics 17.8 (2015), p. 085006 . DOI: $10.1038 /$ ncomms 5185 . 
[13] M. Lostaglio, D. Jennings, and T. Rudolph. "Description of quantum coherence in thermodynamic processes requires constraints beyond free energy". In: $\mathrm{Na}$ ture Communications 6 (2015). DOI: 10.1038/ncomms7383.

[14] M. O. Scully et al. "Extracting work from a single heat bath via vanishing quantum coherence". In: Science 299.5608 (2003), pp. 862-864. DOI: 10.1126/science.1078955.

[15] M. Lostaglio et al. "Quantum Coherence, Time-Translation Symmetry, and Thermodynamics". In: Physical Review X 5 (2 2015), p. 021001. DOI: 10.1103/PhysRevX.5.021001.

[16] F. Binder et al. "Quantum thermodynamics of general quantum processes". In: Physical Review E 91 (3 2015), p. 032119. DOI: 10.1103/PhysRevE.91.032119.

[17] S. Salek and K. Wiesner. "Fluctuations in single-shot $\epsilon$-deterministic work extraction". In: Physical Review A 96 (5 2017), p. 052114. DOI: 10.1103/PhysRevA.96.052114.

[18] H. Tajima and M. Hayashi. "Finite-size effect on optimal efficiency of heat engines". In: Physical Review E 96 (1 2017), p. 012128. DOI: 10.1103/PhysRevE.96.012128.

[19] J. Gemmer, M. Michel, and G. Mahler. Quantum Thermodynamics: Emergence of Thermodynamic Behavior Within Composite Quantum Systems. Lecture Notes in Physics. Springer Berlin Heidelberg, 2009. ISBN: 9783540705109.

[20] J. Roßnagel et al. "Nanoscale Heat Engine Beyond the Carnot Limit". In: Physical Review Letters 112 (3 2014), p. 030602. DOI: 10.1103/PhysRevLett.112.030602.

[21] B. Gardas and S. Deffner. "Thermodynamic universality of quantum Carnot engines". In: Physical Review E 92 (4 2015), p. 042126. DOI: 10.1103/PhysRevE.92.042126.

[22] M. Perarnau-Llobet et al. "Extractable Work from Correlations". In: Physical Review $X \quad 5 \quad(4$ 2015), p. 041011. DOI: 10.1103/PhysRevX.5.041011.
[23] A. M. Alhambra, J. Oppenheim, and C. Perry. "Fluctuating States: What is the Probability of a Thermodynamical Transition?" In: Physical Review X 6.4 (2016), p. 041016. DOI: 10.1103/PhysRevX.6.041016.

[24] A. M. Alhambra et al. "Fluctuating Work: From Quantum Thermodynamical Identities to a Second Law Equality". In: Physical Review X 6.4 (2016), p. 041017. DOI: 10.1103/PhysRevX.6.041017.

[25] H. Spohn and J. L. Lebowitz. "Irreversible thermodynamics for quantum systems weakly coupled to thermal reservoirs". In: Adv. Chem. Phys 38 (1978), pp. 109-142. DOI: $10.1002 / 9780470142578 . \operatorname{ch} 2$.

[26] M. T. Mitchison and P. Potts. "Physical Implementations of Quantum Absorption Refrigerators". In: Thermodynamics in the Quantum Regime: Fundamental Aspects and New Directions. Springer International Publishing, 2018, pp. 149-174. ISBN: 978-3-319-99046-0. DOI: 10.1007/9783-319-99046-0_6.

[27] T. Batalhão et al. "Characterizing Irreversibility in Open Quantum Systems". In: Thermodynamics in the Quantum Regime: Fundamental Aspects and New Directions. Springer International Publishing, 2018, pp. 395-410. ISBN: 978-3-319-99046-0. DOI: 10.1007/978-3-319-99046-0_16.

[28] P. Skrzypczyk et al. "The smallest refrigerators can reach maximal efficiency". In: Journal of Physics A: Mathematical and Theoretical 44.49 (2011), pp. 492002-492008. DOI: $10.1088 \% 2$ F $1751-$ $8113 \% 2 \mathrm{~F} 44 \% 2 \mathrm{~F} 49 \% 2 \mathrm{~F} 492002$.

[29] N. Brunner et al. "Virtual qubits, virtual temperatures, and the foundations of thermodynamics". In: Physical Review E 85 (5 2012), p. 051117. DOI: $10.1103 /$ PhysRevE.85.051117.

[30] M. T. Mitchison et al. "Coherence-assisted single-shot cooling by quantum absorption refrigerators". In: New Journal of Physics 17.11 (2015), p. 115013 . DOI: 10.1088/13672630/17/11/115013. 
[31] N. Brunner et al. "Entanglement enhances cooling in microscopic quantum refrigerators". In: Physical Review E 89 (3 2014), p. 032115. DOI: 10.1103/PhysRevE.89.032115.

[32] R. Alicki. "The quantum open system as a model of the heat engine". In: Journal of Physics A: Mathematical and General 12.5 (1979), pp. L103-L107. DOI: 10.1088/0305$4470 / 12 / 5 / 007$.

[33] D. Gelbwaser-Klimovsky, W. Niedenzu, and G. Kurizki. "Chapter Twelve - Thermodynamics of Quantum Systems Under Dynamical Control". In: Advances In Atomic, Molecular, and Optical Physics. Ed. by Ennio Arimondo, Chun C. Lin, and Susanne F. Yelin. Vol. 64. Academic Press, 2015, pp. 329-407. DOI: 10.1016/bs.aamop.2015.07.002.

[34] R. Kosloff. "Quantum Thermodynamics: A Dynamical Viewpoint". In: Entropy 15.6 (2013), pp. 2100-2128. ISSN: 1099-4300. DOI: $\quad 10.1146 /$ annurev-physchem-040513103724 .

[35] R. Kosloff and A. Levy. "Quantum Heat Engines and Refrigerators: Continuous Devices". In: Annual Review of Physical Chemistry 65.1 (2014). PMID: 24689798, pp. 365-393. DOI: 10.1146/annurevphyschem-040513-103724.

[36] B. Bylicka et al. "Thermodynamic power of non-Markovianity". In: Scientific Reports 6 (2016). DOI: 10.1038/srep27989.

[37] P. Skrzypczyk, A.J. Short, and S. Popescu. "Work extraction and thermodynamics for individual quantum systems". In: Nature Communications 5 (2014). DOI: 10.1038/ncomms5185.

[38] L. Del Rio et al. "The thermodynamic meaning of negative entropy". In: $\mathrm{Na}$ ture 474.7349 (2011), pp. 61-63. DOI: $10.1038 /$ nature10123.

[39] P. Faist et al. "The minimal work cost of information processing". In: $\mathrm{Na}$ ture Communications 6.7669 (2015). DOI: 10.1038/ncomms8669.
[40] S. Deffner and C. Jarzynski. "Information processing and the second law of thermodynamics: An inclusive, Hamiltonian approach". In: Physical Review X 3.4 (2013), p. 041003. DOI: $10.1103 /$ PhysRevX.3.041003.

[41] F. Campaioli et al. "Enhancing the charging power of quantum batteries". In: Physical Review Letters 118.15 (2017), p. 150601. DOI: 10.1103/PhysRevLett.118.150601.

[42] W. Pusz and S. L. Woronowicz. "Passive states and KMS states for general quantum systems". In: Communications in Mathematical Physics 58.3 (1978), pp. 273-290. DOI: $10.1007 / \mathrm{BF} 01614224$.

[43] M. Woods, R. Silva, and J. Oppenheim. "Autonomous Quantum Machines and Finite-Sized Clocks". In: Annales Henri Poincaré (2018). ISSN: 1424-0661. DOI: $10.1007 / \mathrm{s} 00023-018-0736-9$.

[44] H. Wilming, R. Gallego, and J. Eisert. "Axiomatic Characterization of the Quantum Relative Entropy and Free Energy". In: Entropy 19.6 (2017), p. 241. ISSN: 1099-4300. DOI: $10.3390 / \mathrm{e} 19060241$.

[45] M. Esposito, K. Lindenberg, and C. Van den Broeck. "Entropy production as correlation between system and reservoir". In: New Journal of Physics 12.1 (2010), p. 013013. DOI: 10.1088/13672630/12/1/013013.

[46] J. Parrondo, J. M. Horowitz, and T. Sagawa. "Thermodynamics of information". In: Nature Physics 11.2 (2015), pp. 131-139. DOI: 10.1038/nphys3230.

[47] M. P. Müller. "Correlating Thermal Machines and the Second Law at the Nanoscale". In: Physical Review $X 8$ (4 2018), p. 041051. DOI: 10.1103/PhysRevX.8.041051.

[48] E. T. Jaynes. "Information theory and statistical mechanics". In: Physical Review 106.4 (1957), p. 620. DOI: $10.1103 /$ PhysRev.106.620.

[49] N. Ng, M. P. Woods, and S. Wehner. "Surpassing the Carnot efficiency by extracting imperfect work". In: New Journal of Physics 19.11 (2017), p. 113005. DOI: 10.1088/1367-2630/aa8ced. 
[50] R. Renner and S. Wolf. "Smooth Rényi entropy and applications". In: IEEE International Symposium on Information Theory. 2004, pp. 233-233. DOI: 10.1109/ISIT.2004.1365269.

[51] R. Konig, R. Renner, and C. Schaffner. "The operational meaning of min-and maxentropy". In: IEEE Transactions on Information theory 55.9 (2009), pp. 4337-4347. DOI: 10.1109/TIT.2009.2025545.

[52] M. Tomamichel et al. "Leftover hashing against quantum side information". In: IEEE Transactions on Information Theory 57.8 (2011), pp. 5524-5535. DOI: 10.1109/TIT.2011.2158473.

[53] L. Wang, R. Colbeck, and R. Renner. "Simple channel coding bounds". In: 2009 IEEE International Symposium on Information Theory. IEEE. 2009, pp. 1804-1808. DOI: 10.1109/ISIT.2009.5205312.

[54] I. Csiszár. "Generalized cutoff rates and Renyi's information measures". In: IEEE Transactions on information theory 41.1 (1995), pp. 26-34. DOI: 10.1109/18.370121.

[55] O. Shayevitz. "On Rényi measures and hypothesis testing". In: ISIT. 2011, pp. 894898. DOI: 10.1109/ISIT.2011.6034266.

[56] N. Datta. "Min-and max-relative entropies and a new entanglement monotone". In: IEEE Transactions on Information Theory 55.6 (2009), pp. 2816-2826. DOI: 10.1109/TIT.2009.2025545.

[57] F. G. S. L. Brandão and N. Datta. "One-shot rates for entanglement manipulation under non-entangling maps". In: IEEE Transactions on Information Theory 57.3 (2011), pp. 1754-1760. DOI: 10.1109/TIT.2011.2104531.
[58] M. Esposito and C. Van den Broeck. "Second law and Landauer principle far from equilibrium". In: EPL (Europhysics Letters) 95.4 (2011), p. 40004. DOI: 10.1209/0295-5075/95/40004.

[59] M. Lostaglio, M. P. Müller, and M. Pastena. "Stochastic Independence as a Resource in Small-Scale Thermodynamics". In: Physical Review Letters 115 (15 2015), p. 150402. DOI: 10.1103/PhysRevLett.115.150402.

[60] F. Reif. Fundamentals of statistical and thermal physics. McGraw-Hill series in fundamentals of physics. McGraw-Hill, 1965. ISBN: 9781478610052.

[61] R. Uzdin et al. "Markovian heat sources with the smallest heat capacity". In: New Journal of Physics 20.6 (2018), p. 063030. DOI: 10.1088/1367-2630/aac932.

[62] R. Uzdin, A. Levy, and R. Kosloff. "Equivalence of Quantum Heat Machines, and Quantum-Thermodynamic Signatures". In: Physical Review X 5 (3 2015), p. 031044. DOI: 10.1103/PhysRevX.5.031044.

[63] V. Shoup. A Computational Introduction to Number Theory and Algebra. Cambridge University Press, 2009. ISBN: 9780521516440 .

[64] T. M. Cover and J. A. Thomas. Elements of Information Theory 2nd Edition (Wiley Series in Telecommunications and Signal Processing). Wiley-Interscience, 2006. ISBN: 0471241954.

[65] C. Shannon. "A Mathematical Theory of Communication". In: Bell Labs Technical Journal 27 (3 1948), p. 379. DOI: 10.1002/j.1538-7305.1948.tb01338.x.

[66] S. P. Boyd and L. Vandenberghe. Convex Optimization. Cambridge University Press, 2004. ISBN: 9780521833783. 


\section{The maximum efficiency of nano heat engines depends on more than temperature: Supplementary Material}

\section{Contents}

1 Introduction 1

2 Setup 2

2.1 The heat engine model . . . . . . . . . . . . . . . . . . . . . . 2

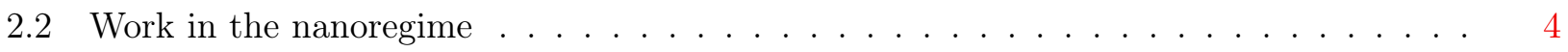

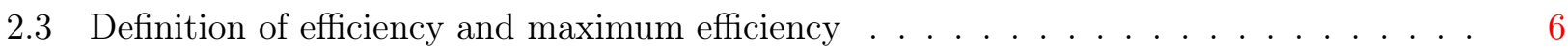

3 Main results $\quad 7$

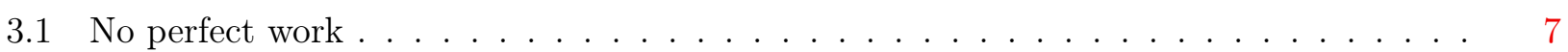

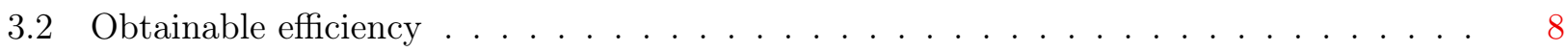

3.3 Comparison to standard free energy results $\ldots \ldots \ldots \ldots \ldots$

4 Proof Overview $\quad 10$

5 Discussions and Conclusion $\quad 13$

$\begin{array}{lr}\text { A The general setting for a heat engine } & 19\end{array}$

B The conditions for thermodynamical state transitions 23

B.1 Second law for macroscopic systems . . . . . . . . . . . . . . . . 23

B.2 Second laws for nanoscopic systems . . . . . . . . . . . . . . . . . . . 23

C Efficiency, maximum efficiency and how to evaluate it 25

C.1 Definition of efficiency and maximum efficiency . . . . . . . . . . . . . 25

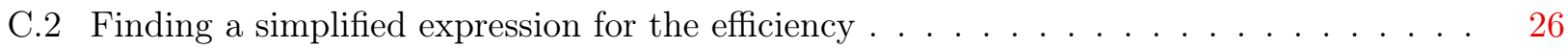

D Efficiency of a heat engine according to macroscopic thermodynamics 26

D.1 Maximum extractable work according to macroscopic law of thermodynamics . . . . 27

D.2 Maximum efficiency for perfect work is Carnot efficiency . . . . . . . . . . . 28

D.3 Maximum efficiency for near perfect work is still Carnot efficiency . . . . . . . . . . . 34

E Efficiency of a nanoscopic quantum heat engine 38

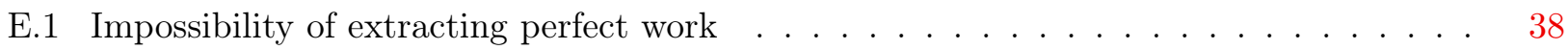

E.2 Efficiency for extracting near perfect work . . . . . . . . . . . . . . . . 39

E.2.1 An explicit expression for $W_{\text {ext }} \ldots \ldots \ldots \ldots \ldots \ldots$

E.2.2 An upper bound for the efficiency . . . . . . . . . . . . . . . . 41

E.2.3 Evaluating near perfect work in the quasi-static heat engine . . . . . . . . . . . 42

E.2.4 The choice of $\varepsilon$ determines the infimum to evaluating $W_{\text {ext }} \ldots \ldots \ldots \ldots$

E.2.5 Solving the infimum for $W_{\text {ext }} \ldots \ldots \ldots \ldots \ldots \ldots \ldots \ldots$

E.2.6 Main results: evaluating the efficiency . . . . . . . . . . . . . . . . 55

E.3 Running the heat engine for many cycles quasi-statically . . . . . . . . . . . . . . . 59

$\begin{array}{lll}\text { F Extensions to the setup } & 61\end{array}$

F.1 Final correlations between battery, cold bath, and machine . . . . . . . . . . . . . 62

F.1.1 Defining the generalized efficiency . . . . . . . . . . . . . . 62

F.1.2 Final correlations do not allow the surpassing of Carnot efficiency . . . . . . . . 63

F.1.3 Achievability of Carnot efficiency still depends on more than temperature . . . 67 
In this Supplementary Material, we detail our findings. Sections A-C are aimed at giving the reader an overview of the important concepts regarding heat engines, and to introduce the quantities of interest. Firstly, in Section A we describe the setup of our heat engine, the systems involved, and how work is extracted and stored. By using this general setup, we then proceed in Section B to introduce conditions for thermodynamical state transitions in a cycle of a heat engine. In Section C, we introduce the formal definition of efficiency, and specify how can this quantity be maximized over a set of free parameters (involving the bath Hamiltonian structure).

After providing these guidelines, we start in Section D to apply the macroscopic law of thermodynamics. We have performed the analysis with the generalization of allowing for an arbitrarily small probability of failure. The results in this section might be familiar and known to the reader, however from a technical perspective, their establishment is helpful for proving our main results (in Section E) about nanoscale systems. In Section E, we apply the recently discovered generalizations of the second law for small quantum systems. The results in Section D and Section E are summarized at the beginning of each section, for the reader to have a concise overview of the distinction between thermodynamics of macroscopic and nanoscopic systems. Finally, in Section F, we show that even when considering a more general setup, these results obtained in Section E remain unchanged.

\section{A The general setting for a heat engine}

A heat engine is a procedure for extracting work from a temperature difference. It comprises of four basic elements: two thermal baths at distinct temperatures $T_{\text {Hot }}$ and $T_{\text {Cold }}$ respectively, a machine, and a battery. The machine interacts with these baths in such a way that utilizes the temperature difference between the two baths to perform work extraction. The extracted work can then be transferred and stored in the battery, while the machine returns to its original state.

In this section, we describe a fully general setup, where all involved systems and changes in energy are accounted for explicitly. Let us begin with the total Hamiltonian

$$
\hat{H}_{t}=\hat{H}_{\mathrm{Cold}}+\hat{H}_{\mathrm{Hot}}+\hat{H}_{\mathrm{M}}+\hat{H}_{\mathrm{W}}
$$

where the indices Hot, Cold, M, W represent a hot thermal bath (Hot), a cold thermal bath (Cold), a machine $(\mathrm{M})$, and a battery $(\mathrm{W})$ respectively. Let us also consider an initial state

$$
\rho_{\text {ColdHotMW }}^{0}=\tau_{\text {Cold }}^{0} \otimes \tau_{\text {Hot }}^{0} \otimes \rho_{\mathrm{M}}^{0} \otimes \rho_{\mathrm{W}}^{0} .
$$

The state $\tau_{\text {Hot }}^{0}\left(\tau_{\text {Cold }}^{0}\right)$ is the initial thermal state at temperature $T_{\text {Hot }}\left(T_{\text {Cold }}\right)$, corresponding to the hot (cold) bath Hamiltonians $\hat{H}_{\text {Hot }}, \hat{H}_{\text {Cold }}$. More generally, we have the following definition

Definition 3. (Thermal state) Given any Hamiltonian $\hat{H}$ and temperature $T$, the thermal state is defined as $\tau=\frac{1}{\operatorname{tr}\left(e^{-\hat{H} / k_{B} T}\right)} e^{-\hat{H} / k_{B} T}$. For notational convenience, we shall often use inverse temperatures, defined as $\beta_{h}:=1 / k_{B} T_{\text {Hot }}$ and $\beta_{c}:=1 / k_{B} T_{\text {Cold }}$ where $k_{B}$ is the Boltzmann constant.

We will assume throughout that $T_{\text {Cold }}<T_{\text {Hot }}$. This is what is meant by "hot"; namely that it is at a higher temperature than the "cold" bath. The initial machine $\left(\rho_{\mathrm{M}}^{0}, \hat{H}_{\mathrm{M}}\right)$ can be chosen arbitrarily, as long as its final state is preserved (and therefore the machine acts like a catalyst).

The aim is to achieve a final reduced state $\rho_{\text {ColdHotMw }}^{1}$, such that

$$
\rho_{\text {ColdMW }}^{1}=\operatorname{tr}_{\text {Hot }}\left(\rho_{\text {ColdHotMW }}^{1}\right)=\rho_{\text {Cold }}^{1} \otimes \rho_{\mathrm{M}}^{1} \otimes \rho_{\mathrm{W}}^{1},
$$

where $\rho_{\mathrm{M}}^{1}=\rho_{\mathrm{M}}^{0}$, i.e. the machine is preserved, and $\rho_{\text {Cold }}^{1}, \rho_{\mathrm{W}}^{1}$ are the final states of the cold bath and battery. In Section F, we will consider the case in which there are correlations between the final state of the cold bath, hot bath, battery and or machine. We will find that the correlations do not 
change our results. For any bipartite state $\rho_{\mathrm{AB}}$, we use the notation of reduced states $\rho_{\mathrm{A}}:=\operatorname{tr}_{\mathrm{B}}\left(\rho_{\mathrm{AB}}\right)$, $\rho_{\mathrm{B}}:=\operatorname{tr}_{\mathrm{A}}\left(\rho_{\mathrm{BA}}\right)$.

Finally, we describe the battery such that the state transformation from $\rho_{\text {ColdHotMw }}^{0}$ to $\rho_{\text {ColdHotMw }}^{1}$ stores work in the battery. This is done as follows: consider the battery which has a Hamiltonian (written in its diagonal form)

$$
\hat{H}_{\mathrm{W}}:=\sum_{i=1}^{n_{\mathrm{W}}} E_{i}^{\mathrm{W}}\left|E_{i}\right\rangle\left\langle\left. E_{i}\right|_{\mathrm{W}},\right.
$$

where $\left\{E_{i}^{\mathrm{W}} \in \mathbb{R}\right\}_{i=1}^{n_{\mathrm{W}}}$ are arbitrary while $n_{\mathrm{W}} \in \mathbb{N}^{+}$is an arbitrarily large fixed integer. For some parameter $\varepsilon \in[0,1)$, we consider the initial and final states of the battery to be

$$
\begin{aligned}
& \rho_{\mathrm{W}}^{0}=\left|E_{j}\right\rangle\left\langle\left. E_{j}\right|_{\mathrm{W}}\right. \\
& \rho_{\mathrm{W}}^{1}=(1-\varepsilon)\left|E_{k}\right\rangle\left\langle\left. E_{k}\right|_{\mathrm{W}}+\varepsilon \mid E_{j}\right\rangle\left\langle\left. E_{j}\right|_{\mathrm{W}}\right.
\end{aligned}
$$

respectively. The parameter $W_{\text {ext }}$ is defined as the energy difference

$$
W_{\text {ext }}:=E_{k}^{\mathrm{W}}-E_{j}^{\mathrm{W}} .
$$

where we define $E_{k}^{\mathrm{W}}>E_{j}^{\mathrm{W}}$ such that $W_{\text {ext }}>0$. We refer to the parameter $\varepsilon$ as the probability of failure of work extraction. Note that $\varepsilon$ in Eq. (42) is also the trace distance

$$
d(\rho, \sigma)=\frac{1}{2}\|\rho-\sigma\|_{1}=\frac{1}{2} \operatorname{tr}\left[\sqrt{(\rho-\sigma)^{\dagger}(\rho-\sigma)}\right]
$$

between $\rho_{\mathrm{W}}^{1}$ and $\left|E_{k}\right\rangle\left\langle\left. E_{k}\right|_{\mathrm{W}}\right.$. In Section $\mathrm{F}$, we will generalize this definition to include all final states of the battery $\rho_{\mathrm{W}}^{1}$, which are a trace distance $\varepsilon$ from the ideal final battery state $\left|E_{k}\right\rangle\left\langle\left. E_{k}\right|_{\mathrm{W}}\right.$. We show that our findings regarding the achievability of C.E. remains unchanged.

Throughout our analysis, we deal with two distinct scenarios of work extraction as defined below.

Definition 4. (Perfect work) An amount of work extracted $W_{\text {ext }}$ is referred to as perfect work when $\varepsilon=0$.

The next definition of work involves a condition regarding the von Neumann entropy of the final battery state. Let $\Delta S$ be the von Neumann entropy of the final battery state. When the initial state $\rho_{\mathrm{W}}^{0}$ is pure, we have

$$
\Delta S:=-\operatorname{tr}\left(\rho_{\mathrm{W}}^{1} \ln \rho_{\mathrm{W}}^{1}\right) .
$$

When the final battery state is given by Eq. (42), its probability distribution has its support on a two-dimensional subspace of the battery system, this definition also coincides with the binary entropy of $\varepsilon$,

$$
\mathrm{h}_{2}(\varepsilon)=-\varepsilon \ln \varepsilon-(1-\varepsilon) \ln (1-\varepsilon)=\Delta S .
$$

We will see that no heat-engine can actually achieve the value of Carnot efficiency exactly, but moreover, that under certain conditions; some achieve it as a limiting process. ${ }^{8}$ For this reason, it is convenient to introduce the notion of a partially ordered set of heat engines. Roughly speaking, we will say later that a heat engine can achieve the Carnot efficiency when a heat engine in the closure of the set can achieve said efficiency. Let $\mathcal{S}^{\text {H.E. }}=\left\{\left(W_{\text {ext }}, \varepsilon\right)\right\}$ denote a partially ordered set ${ }^{9}$ of heat engines with extracted work $W_{\text {ext }}$ and corresponding failure parameter $\varepsilon$, introduced above.

Definition 5. (Near perfect work) We say that a partially ordered set of heat engines $\mathcal{S}^{\text {H.E. }}$ (introduced above) can achieve near perfect work when

\footnotetext{
${ }^{8}$ This subtlety is not unique to nano scale sized systems, but moreover is also true at the macroscopic level. In the standard formulation of Carnot's famous results about heat engine efficiency, the Carnot efficiency can only be achieved in the so-called "quasi-static limit".

${ }^{9}$ The partial order is given by the condition $W_{\text {ext }} \leq W_{\text {ext }}^{\prime}$ for two set elements $\left(W_{\text {ext }}, \varepsilon\right),\left(W_{\text {ext }}^{\prime}, \varepsilon^{\prime}\right) \in \mathcal{S}^{\text {H.E. }}$.
} 


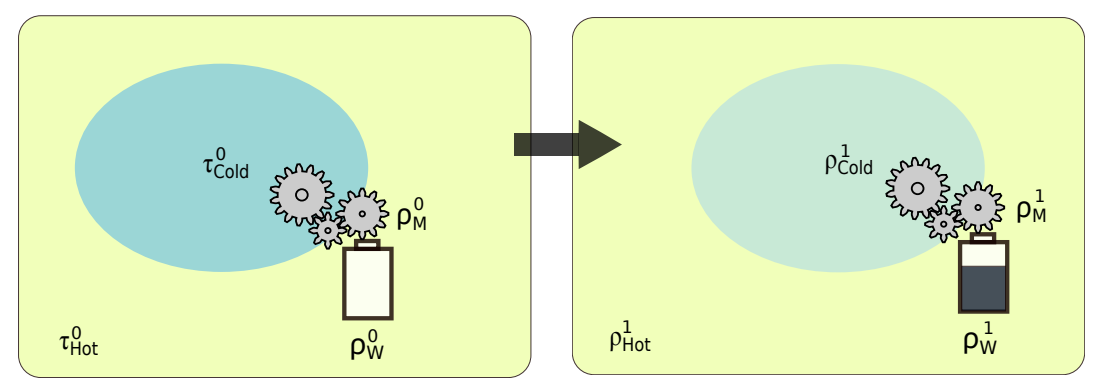

Figure 5: The setting of a working heat engine.

1) $0<\varepsilon \leq l$, for some fixed $l<1$ and

2) For all $1>p>0$ there exists a non trivial subset $\mathcal{S}_{p}^{\text {H.E. }} \subset \mathcal{S}^{\text {H.E. }}$ such that when $\left(W_{\text {ext }}, \varepsilon\right) \in \mathcal{S}_{p}^{\text {H.E. }}$,

$$
0<\frac{\Delta S}{W_{\text {ext }}}<p
$$

where recall $\Delta S=\Delta S(\varepsilon)$ [see Eq. (46)].

Note that when this definition is used in lemmas and theorems, the precise type of "heat engine" will be specified, e.g. heat engines satisfying the macroscopic laws of thermodynamics (which are defined later). In the main text, we have provided a detailed discussion regarding the physical meaning of perfect work and near perfect work, and the necessity for considering these quantities. In particular, it is discussed how it can only be achieved as a limiting process. Why we are interested in such limits will become apparent when we discuss the macroscopic case, even before we derive the efficiency in the nano regime. As we will see later in the proof to Lemma 5, 1) and 2) in Def. 5 are both satisfied if and only if

$$
\lim _{\varepsilon \rightarrow 0^{+}} \frac{\Delta S}{W_{\text {ext }}}=0
$$

Since the initial state $\rho_{\text {ColdHotMW }}^{0}$ is diagonal in the energy eigenbasis, and since catalytic thermal operations do not create coherences between energy eigenstates, therefore $\rho_{\text {ColdMw }}^{1}$ has to be diagonal in the energy eigenbasis. Furthermore, (as already stated above) in Section F, we extend the setup to include correlation in the final state between the battery, cold bath and machine and more general final battery states.

Note that in our model we allow the battery to have arbitrarily many (but finite) eigenvalues. One can compare this to the two-dimensional battery used in [7], referred to as the wit. Having a minimal dimension, the wit is a conceptually very useful tool to visualize work extraction. However, it has the disadvantage that the energy spacing, i.e. the amount of work to be extracted, has to be known a priori to the work being extracted in order to tune the energy gap of the wit. The more general battery, which we describe in Eq. (40), requires a higher system dimension, but has the advantage that it can form a quasi-continuum and thus effectively any amount of work (i.e. any $W_{\text {ext }}>0$ ) can be stored in it without prior knowledge of the work extraction process. We will see that our results are independent of $n_{\mathrm{W}} \geq 2$.

To summarize, so far we have made the following minimal assumptions:

(A.1) Product state: There are no initial nor final correlations between the cold bath, machine and battery. Initial correlations we assume do not exist, since each of the initial systems are brought independently into the process. This is an advantage of our setup, since if one assumed initial coherence, one would then have to use unknown resources to generate them in the first place. We later also show that correlations between the final cold bath and battery do not provide improvements in maximum extractable work or efficiency. 
(A.2) Perfect cyclicity: The machine undergoes a cyclic process, i.e. $\rho_{\mathrm{M}}^{0}=\rho_{\mathrm{M}}^{1}$.

(A.3) Isolated quantum system: The heat engine as a whole, is isolated from and does not interact with the world. This assumption ensures that all possible resources in a work extraction process has been accounted for.

(A.4) Finite dimension: The Hilbert space associated with $\rho_{\text {ColdHotMw }}^{0}$ is finite dimensional but can be arbitrarily large. Moreover, the Hamiltonians $\hat{H}_{\text {Cold }}, \hat{H}_{\mathrm{Hot}}, \hat{H}_{\mathrm{M}}$ and $\hat{H}_{\mathrm{W}}$ all have bounded pure point spectra, meaning that these Hamiltonians have eigenvalues which are bounded.

After defining the set of allowed operations, and describing the desired state transformation process, one can then ask: what conditions should be fulfilled such that there exists a hot bath $\left(\tau_{\text {Hot }}^{0}, \hat{H}_{\text {Hot }}\right)$, and a machine $\left(\rho_{\mathrm{M}}^{0}, \hat{H}_{\mathrm{M}}\right)$ such that $\rho_{\text {ColdW }}^{0} \rightarrow \rho_{\text {ColdW }}^{1}$ is possible? Throughout this document we use " $\rightarrow$ " to denote a state transition via catalytic thermal operations.

In Section D, by assuming the macroscopic law of thermodynamics governs the heat engine, we derive the efficiency of a heat engine, and verify the long known Carnot efficiency as the optimal efficiency. We do this for both cases where $\varepsilon=0$ and when $\varepsilon$ is arbitrarily small. In Section E, we analyze the same problem under recently derived second laws, which hold for small quantum systems. We show that these new second laws lead to fundamental differences to the efficiency of a heat engine.

Throughout our analysis, a particular notion that describes thermodynamical transitions will be important towards achieving maximum efficiency. We therefore define this technical term, which will be used throughout the manuscript.

Definition 6. (Quasi-static) A heat engine is quasi-static if the final state of the cold bath is a thermal state and its inverse temperature $\beta_{f}$ only differs infinitesimally from the initial cold bath temperature, i.e. $\beta_{f}=\beta_{c}-g$, where $0<g \ll 1$.

Since throughout this analysis we frequently deal with arbitrarily small paramaters $\varepsilon, g$, we also introduce beforehand the notation of order function $\Theta(x), o(x)$, which denotes the growth of a function.

Definition 7. (Big $\Theta$, small o notation [63]) Consider two real-valued functions $P(x), Q(x)$. We say that

1. $P(x)=\Theta(Q(x))$ in the limit $x \rightarrow a$ iff there exists $c_{1}, c_{2}>0$ and $\delta>0$ such that for all $|x-a| \leq \delta$, $c_{1} \leq\left|\frac{P(x)}{Q(x)}\right| \leq c_{2}$.

2. $P(x)=o(Q(x))$ in the limit $x \rightarrow a$ iff there exists $c_{3} \geq 0$ such that $\lim _{x \rightarrow a}\left|\frac{P(x)}{Q(x)}\right|=c_{3}$.

Remark 1. In Def.7, if the limit of $x$ is unspecified, by default we take $a=0$. In [63], these order terms were only defined for $x \rightarrow \infty$. However, choosing a general limit $x \rightarrow a$ can be done by simply defining the variable $x^{\prime}=1 /(x-a)$, and $x \rightarrow a^{+}$is the same as taking $x^{\prime} \rightarrow \infty$.

We also list a few properties of these functions here for $x \rightarrow 0$, which will help us throughout the proof:

a) For any $c \neq 0, \Theta(c \cdot P(x))=\Theta(P(x))$.

b) For any functions $P_{1}(x)$ and $P_{2}(x), \Theta\left(P_{1}(x)\right)+\Theta\left(P_{2}(x)\right)=\Theta\left(\max \left\{\left|P_{1}(x)\right|,\left|P_{2}(x)\right|\right\}\right)$.

c) For any functions $P_{1}(x)$ and $P_{2}(x), \Theta\left(P_{1}(x)\right) \cdot \Theta\left(P_{2}(x)\right)=\Theta\left(P_{1}(x) P_{2}(x)\right)$.

d) For any functions $P_{1}(x)$ and $P_{2}(x), \Theta\left(P_{1}(x)\right) / \Theta\left(P_{2}(x)\right)=\Theta\left(P_{1}(x) / P_{2}(x)\right)$.

Definition 6 has two direct implications for a quasi-static heat engine:

(i) The temperature of the final state of the cold bath $T_{f}$, only increases w.r.t. its initial temperature by an infinitesimal amount, i.e. $T_{f}=T_{\text {Cold }}+T_{\text {Cold }}^{2} g+\Theta\left(g^{2}\right)$.

(ii) The amount of work extracted is infinitesimal: as we shall see later, the extractable perfect and near perfect work $W_{\text {ext }}>0$ (see Defs. (4), (5)) is of order $\Theta(g)$. This follows from using Eq. (82) for the case where $\rho_{\text {Cold }}^{1}$ is a thermal state with inverse temperature $\beta_{f}=\beta_{c}-g$, and calculating the Taylor expansion of $W_{\text {ext }}$ about $g=0$. 


\section{B The conditions for thermodynamical state transitions}

In this section, we state the laws which govern the transitions from initial, $\rho_{\text {ColdHotMw }}^{0}$ to final, $\rho_{\text {ColdHotMw }}^{1}$ states for one cycle of our heat engine. By applying these laws, the amount of extractable work $W$ ext can be quantified and expressed as a function of the cold bath. We distinguish between two cases, the standard macroscopic regime, and the quantum regime.

\section{B.1 Second law for macroscopic systems}

The cold bath, machine and battery form a closed but not isolated thermodynamic system. This means only heat exchange (and not mass exchange) occurs between these systems and the hot bath. Therefore, a transition from $\rho_{\text {ColdMw }}^{0}$ to $\rho_{\text {ColdMw }}^{1}$ will be possible if and only if the non-equilibrium free energy, $F$ does not increase

$$
F\left(\rho_{\text {ColdMW }}^{0}\right) \geq F\left(\rho_{\text {ColdMW }}^{1}\right),
$$

where

$$
F(\rho):=\langle\hat{H}\rangle_{\rho}-\frac{1}{\beta} S(\rho),
$$

and $S(\rho):=-\operatorname{tr}(\rho \ln \rho)$ and $\langle\hat{H}\rangle_{\rho}:=\operatorname{tr}(\hat{H} \rho)$ being the entropy and the mean energy of state $\rho$ respectively. Throughout the manuscript, whenever the state is a thermal state at temperature $\beta$, we use the shorthand notation $\left\langle\hat{H}_{\text {Cold }}\right\rangle_{\beta}$ and $S(\beta)$.

The non-equilibrium free energy bears a close relation to the relative entropy,

$$
D(\rho \| \sigma)=\operatorname{tr}(\rho \ln \rho)-\operatorname{tr}(\rho \ln \sigma) .
$$

Whenever $\rho$ and $\sigma$ are diagonal in the same basis, the relative entropy can be written as

$$
D(\rho \| \sigma)=\sum_{i} p_{i} \ln \frac{p_{i}}{q_{i}}
$$

where $p_{i}, q_{i}$ are the eigenvalues of $\rho$ and $\sigma$ respectively. Now, for any Hamiltonian $\hat{H}$, consider $\tau_{\beta}=$ $\mathrm{e}^{-\beta \hat{H}} / Z_{\beta}$, which is the thermal state at some inverse temperature $\beta$, with partition function $Z_{\beta}=$ $\operatorname{tr}\left[\mathrm{e}^{-\beta \hat{H}}\right]$, and denote its eigenvalues as $q_{i}$. Then for any diagonal state $\rho$ with eigenvalues $p_{i}$, and denoting $\left\{E_{i}\right\}_{i}$ as the eigenvalues of $\hat{H}$,

$$
D\left(\rho \| \tau_{\beta}\right)=\sum_{i} p_{i} \ln \frac{p_{i}}{q_{i}}=-S(\rho)+\sum_{i} p_{i}\left(\beta E_{i}+\ln Z_{\beta}\right)=\beta F(\rho)+\ln Z_{\beta}
$$

This implies that

$$
F(\rho)=\frac{1}{\beta}\left[D\left(\rho \| \tau_{\beta}\right)-\ln Z_{\beta}\right] .
$$

In Section D we will solve Eq. (49) in order to evaluate the maximum efficiency.

\section{B.2 Second laws for nanoscopic systems}

In the microscopic quantum regime, where only a few quantum particles are involved, it has been shown that macroscopic thermodynamics is not a complete description of thermodynamical transitions. More precisely, not only the non-equilibrium free energy, but a whole other family of generalized free energies have to decrease during a state transition [7]. This places further constraints on whether a particular transition is allowed. In particular, these laws also give necessary and sufficient conditions, when a system with initial state $\rho_{\text {ColdW }}^{0}$ can be transformed to final state $\rho_{\text {ColdW }}^{1}$ (both diagonal in the energy eigenbasis), with the help of any catalyst/machine which is returned to its initial state after the process.

Formally, these laws correspond to the following case: A transition from the initial state $\rho_{\text {ColdMw }}^{0}$ to the final state $\rho_{\text {ColdMW }}^{1}$, is possible iff there exists an energy-preserving unitary $U(t)$ on the global 
system, (i.e. a unitary that obeys $\left[U(t), \hat{H}_{\text {ColdHotMw }}\right]=0$ ), where states $\rho_{\text {ColdMw }}^{0}, \rho_{\text {ColdMw }}^{1}$ are of the form Section described in A (i.e. the state of the machine $\rho_{\mathrm{M}}^{0}$ is preserved). If $\left(\tau_{\mathrm{Hot}}^{0}, \hat{H}_{\mathrm{Hot}}\right)$ and $\left(\rho_{\mathrm{M}}^{0}, \hat{H}_{\mathrm{M}}\right)$ can be arbitrarily chosen, these correspond to the set of catalytic thermal operations [7] one can perform on the joint state ColdW. This implies that the cold bath is used as a resource state.

We can apply these second laws to our scenario by associating the catalyst with $\rho_{\mathrm{M}}^{0}$, and considering the state transition $\rho_{\mathrm{W}}^{0} \otimes \tau_{\text {Cold }}^{0} \rightarrow \rho_{\mathrm{W}}^{1} \otimes \rho_{\text {Cold }}^{1}$ as described in Section A. Note that the initial state $\rho_{\mathrm{W}}^{0} \otimes \tau_{\text {Cold }}^{0}$ is block-diagonal in the energy eigenbasis (for the battery by our choice, and for the cold bath because it is a thermal state). By catalytic thermal operations, the final state is also blockdiagonal in the energy eigenbasis. Furthermore, according to the second laws in [7], the transition from $\rho_{\mathrm{W}}^{0} \otimes \tau_{\text {Cold }}^{0} \rightarrow \rho_{\mathrm{W}}^{1} \otimes \rho_{\text {Cold }}^{1}$ is then possible iff

$$
F_{\alpha}\left(\tau_{\text {Cold }}^{0} \otimes \rho_{\mathrm{W}}^{0}, \tau_{\text {ColdW }}^{h}\right) \geq F_{\alpha}\left(\rho_{\text {Cold }}^{1} \otimes \rho_{\mathrm{W}}^{1}, \tau_{\text {ColdW }}^{h}\right) \quad \forall \alpha \geq 0,
$$

where $\tau_{\text {ColdW }}^{h}$ is the thermal state of the system at temperature $T_{\text {Hot }}$ of the surrounding bath. The quantity $F_{\alpha}(\rho, \sigma)$ for $\alpha \geq 0$ corresponds to a family of free energies defined in [7], which can be written in the form

$$
F_{\alpha}(\rho, \tau)=\frac{1}{\beta_{h}}\left[D_{\alpha}(\rho \| \tau)-\ln Z_{h}\right],
$$

where $D_{\alpha}(\rho \| \tau)$ are known as $\alpha$-Rényi divergences. Sometimes we will use the short hand $F_{\infty}:=$ $\lim _{\alpha \rightarrow \infty} F_{\alpha}$. On occasion, we will refer to a particular transition as being possible/impossible according to the $F_{\alpha}$ free energy constraint. By this, we mean that for that particular value of $\alpha$ and transition, Eq. (55) is satisfied/not satisfied. The $\alpha$-Rényi divergences can be defined for arbitrary quantum states, giving us necessary (but insufficient) second laws for state transitions [7, 13]. However, since we are analyzing states which are diagonal in the same eigenbasis, the Rényi divergences can be simplified to

$$
D_{\alpha}(\rho \| \tau)=\frac{1}{\alpha-1} \ln \sum_{i} p_{i}^{\alpha} q_{i}^{1-\alpha}
$$

where $p_{i}, q_{i}$ are the eigenvalues of $\rho$ and the state $\tau$. The cases $\alpha=0$ and $\alpha \rightarrow 1$ are defined by continuity, namely

$$
\begin{aligned}
& D_{0}(\rho \| \tau)=\lim _{\alpha \rightarrow 0^{+}} D_{\alpha}(\rho \| \tau)=-\ln \sum_{i: p_{i} \neq 0} q_{i}, \\
& D_{1}(\rho \| \tau)=\lim _{\alpha \rightarrow 1} D_{\alpha}(\rho \| \tau)=\sum_{i} p_{i} \ln \frac{p_{i}}{q_{i}}
\end{aligned}
$$

and we also define $D_{\infty}$ as

$$
D_{\infty}(\rho \| \tau)=\lim _{\alpha \rightarrow \infty^{+}} D_{\alpha}(\rho \| \tau)=\ln \max _{i} \frac{p_{i}}{q_{i}} .
$$

The quantity $D_{1}(\rho \| \tau)$ coincides with $D(\rho \| \tau)$, as we have defined in Eq. (51), and evaluated in Eq. (52) for diagonal states. We will often use this convention. Furthermore, since we are considering initial states which are block-diagonal in the energy eigenbasis, these generalized second laws are both necessary and sufficient conditions for state transformations. Therefore, in Section E.2.1 we will solve Eq. (55) explicitly to find an expression for $W_{\text {ext }}$ with the ultimate goal of evaluating the maximum efficiency in this regime.

The reader should note that for both Section B.1 and B.2, the conditions for state transformation place upper bounds on the quantity $W_{\text {ext }}$. In particular, this allows us to express the maximum values $W_{\text {ext }}$ can take (such that the joint state transformation of cold bath and battery is possible) in terms of quantities related to the cold bath, and the error probability $\varepsilon$. It is also worth comparing the conditions for state transformation in Section B.1 and B.2, which are stated in Eqs. (49) and (55). In particular, Eq. (49) is but a particular instance of Eq. (55), and therefore the nanoscopic second laws always place a stronger upper bound on $W_{\text {ext }}$ compared to the macroscopic second law. 


\section{Efficiency, maximum efficiency and how to evaluate it}

The central quantity of interest in this article is the efficiency of heat engines. Since we have already introduced the notion of a heat engine in Section A, and the rules which govern the possibility of thermodynamical transitions of one cycle of a heat engine in Section B, it is timely to define the efficiency. After defining this quantity, we demonstrate how to go about calculating its maximum value under different conditions, such as for perfect work, near perfect work, in both the macroscopic and nanoscopic regimes. This will prepare the scene for Sections D and E, where we evaluate the maximum efficiency more explicitly.

\section{C.1 Definition of efficiency and maximum efficiency}

As stated in the main text, the efficiency of a particular heat engine (recall that a heat engine is defined by its initial and final states $\rho_{\text {ColdHotMW }}^{0}, \rho_{\text {ColdHotMW }}^{1}$ as described in Section A) is defined as

$$
\eta:=\frac{W_{\text {ext }}}{\Delta H}
$$

where $W_{\text {ext }}$ is the amount of work extracted which is defined in Eq. (43), and $\Delta H$ is the amount of mean energy drawn from the hot bath, namely $\Delta H:=\operatorname{tr}\left(\hat{H}_{\text {Hot }} \rho_{\text {Hot }}^{0}\right)-\operatorname{tr}\left(\hat{H}_{\text {Hot }} \rho_{\text {Hot }}^{1}\right)$, where $\rho_{\text {Hot }}^{1}$ is the reduced state of the hot bath.

Now, consider the set of conditions on state transformations given by Eq. (55) for nanoscale systems. As discussed in Section B, these conditions place a restriction on the range of values $W_{\text {ext }}$ can take. Therefore, for any fixed $\rho_{\text {Cold }}^{1}$, we define $\eta^{\text {nano }}\left(\rho_{\text {Cold }}^{1}\right)$ as the maximum achievable efficiency as a function of the final state of the cold bath. More precisely,

$$
\begin{aligned}
& \eta^{\text {nano }}\left(\rho_{\text {Cold }}^{1}\right) \\
& =\sup _{W_{\text {ext }}} \eta\left(\rho_{\text {Cold }}^{1}\right) \quad \text { subject to } \quad F_{\alpha}\left(\rho_{\mathrm{W}}^{0} \otimes \tau_{\text {Cold }}^{0}, \tau_{\text {ColdW }}^{h}\right) \geq F_{\alpha}\left(\rho_{\mathrm{W}}^{1} \otimes \rho_{\text {Cold }}^{1}, \tau_{\text {ColdW }}^{h}\right) \quad \forall \alpha \geq 0 .
\end{aligned}
$$

In Eq. (62), we have written the quantity in Eq. (61) as $\eta=\eta\left(\rho_{\text {Cold }}^{1}\right)$ to remind ourselves of its explicit final cold bath state dependency. Therefore, the maximum efficiency will correspond to maximizing over the final state of the cold bath:

$$
\eta_{\max }=\sup _{\rho_{\text {Cold }}^{1} \in \mathcal{S}} \eta^{\text {nano }}\left(\rho_{\text {Cold }}^{1}\right)
$$

where $\mathcal{S}$ is the space of all quantum states in $\mathcal{H}_{\text {Cold }}$. By analyzing this quantity in Section E, we show that perfect work cannot be extracted. Therefore, when we calculate the maximization in Eq. (64) we will consider near perfect work (see Def. 5).

In the macro regime, we have to satisfy a less stringent requirement, namely the macroscopic second law of thermodynamics. And hence we have that for fixed $\rho_{\text {Cold }}^{1}, \eta^{\operatorname{mac}}\left(\rho_{\text {Cold }}^{1}\right)$ is the maximum efficiency as a function of $\rho_{\text {Cold }}^{1}$

$$
\begin{aligned}
\eta^{\text {mac }}\left(\rho_{\text {Cold }}^{1}\right)=\sup _{W_{\text {ext }}} \eta\left(\rho_{\text {Cold }}^{1}\right) \quad \text { subject to } \quad F\left(\rho_{\text {ColdMW }}^{0}\right) \geq F\left(\rho_{\text {ColdMW }}^{1}\right) \\
\text { and } \operatorname{tr}\left(\hat{H}_{t} \rho_{\text {ColdHotMW }}^{0}\right)=\operatorname{tr}\left(\hat{H}_{t} \rho_{\text {ColdHotMW }}^{1}\right),
\end{aligned}
$$

where $\hat{H}_{t}$ is defined in Eq. (37). Similarly to the nanoscale setting, the maximum efficiency is

$$
\eta_{\max }=\sup _{\rho_{\text {Cold }}^{1} \in \mathcal{S}} \eta^{\text {mac }}\left(\rho_{\text {Cold }}^{1}\right) .
$$

We can also define the maximum quasi-static efficiencies for the macro and nano scale. The maximum efficiency of a quasi-static heat engine (see Def. 6), is

$$
\begin{aligned}
& \eta_{\text {max }}^{\text {stat }}=\lim _{g \rightarrow 0^{+}} \eta^{\text {nano }}(\tau(g)), \\
& \eta_{\max }^{\text {stat }}=\lim _{g \rightarrow 0^{+}} \eta^{\text {mac }}(\tau(g)),
\end{aligned}
$$


for the nanoscopic and macroscopic cases respectively. $\tau(g) \in \mathcal{H}_{\text {Cold }}$ is the thermal state with Hamiltonian $\hat{H}_{\text {Cold }}$ at temperature $\beta_{f}=\beta_{c}-g$ and $\eta^{\text {nano }}, \eta^{\text {mac }}$ are defined in Eqs. (62) and (65) respectively. Since we can extract perfect and near perfect work in the macroscopic setting, we will derive the efficiency for both cases in Section D.

\section{C.2 Finding a simplified expression for the efficiency}

We can find a more useful expression for $\Delta H$ appearing in Eq. (61). This can be obtained by observing that since only energy preserving operations are allowed, we have

$$
\operatorname{tr}\left(\hat{H}_{t} \rho_{\text {ColdHotMW }}^{0}\right)=\operatorname{tr}\left(\hat{H}_{t} \rho_{\text {ColdHotMW }}^{1}\right),
$$

where $\hat{H}_{t}=\hat{H}_{\mathrm{Hot}}+\hat{H}_{\mathrm{Cold}}+\hat{H}_{\mathrm{M}}+\hat{H}_{\mathrm{W}}$. Since the Hamiltonian does not contain interaction terms between these systems, the mean energy depends only on the reduced states of each system. Mathematically, it means that Eq. (70) can be written as

$$
\begin{array}{r}
\operatorname{tr}\left(\hat{H}_{\text {Hot }} \rho_{\text {Hot }}^{0}\right)+\operatorname{tr}\left(\hat{H}_{\text {Cold }} \rho_{\text {Cold }}^{0}\right)+\operatorname{tr}\left(\hat{H}_{\mathrm{M}} \rho_{\mathrm{M}}^{0}\right)+\operatorname{tr}\left(\hat{H}_{\mathrm{W}} \rho_{\mathrm{W}}^{0}\right)= \\
\operatorname{tr}\left(\hat{H}_{\text {Hot }} \rho_{\text {Hot }}^{1}\right)+\operatorname{tr}\left(\hat{H}_{\text {Cold }} \rho_{\text {Cold }}^{1}\right)+\operatorname{tr}\left(\hat{H}_{\mathrm{M}} \rho_{\mathrm{M}}^{1}\right)+\operatorname{tr}\left(\hat{H}_{\mathrm{W}} \rho_{\mathrm{W}}^{1}\right) .
\end{array}
$$

Also, note that since $\rho_{\mathrm{M}}^{0}=\rho_{\mathrm{M}}^{1}$, therefore $\operatorname{tr}\left(\hat{H}_{\mathrm{M}} \rho_{\mathrm{M}}^{0}\right)=\operatorname{tr}\left(\hat{H}_{\mathrm{M}} \rho_{\mathrm{M}}^{1}\right)$. This implies that we have

$$
\Delta H=\Delta C+\Delta W,
$$

where

$$
\Delta C:=\operatorname{tr}\left[\hat{H}_{\text {Cold }} \rho_{\text {Cold }}^{1}\right]-\operatorname{tr}\left[\hat{H}_{\text {Cold }} \tau_{\text {Cold }}^{c}\right]
$$

and

$$
\Delta W:=\operatorname{tr}\left(\hat{H}_{\mathrm{W}} \rho_{\mathrm{W}}^{1}\right)-\operatorname{tr}\left(\hat{H}_{\mathrm{W}} \rho_{\mathrm{W}}^{0}\right)
$$

are the change in average energy of the cold bath and battery. We can thus write Eq. (61) as

$$
\eta=\frac{W_{\mathrm{ext}}}{\Delta W+\Delta C}
$$

Furthermore, from Eqs. (41), (42), (43) and (75), we have $\Delta W=(1-\varepsilon) W_{\text {ext }}$, and hence we can write the inverse efficiency as

$$
\eta^{-1}\left(\rho_{\text {Cold }}^{1}\right)=1-\varepsilon+\frac{\Delta C\left(\rho_{\text {Cold }}^{1}\right)}{W_{\text {ext }}\left(\rho_{\text {Cold }}^{1}\right)},
$$

where we have made explicit the $\rho_{\text {Cold }}^{1}$ dependency. We already know from the setting that $\rho_{\text {Cold }}^{0}$ is thermal. If $\rho_{\text {Cold }}^{1}$ is also a thermal state at some temperature $\beta$ according to the cold bath Hamiltonian $\hat{H}_{\text {Cold }}$, we will sometimes use the shorthand notation $\eta(\beta)$ for $\eta\left(\rho_{\text {Cold }}^{1}\right)$ and $\Delta W(\beta), \Delta C(\beta)$ for $\Delta W\left(\rho_{\text {Cold }}^{1}\right), \Delta C\left(\rho_{\text {Cold }}^{1}\right)$ respectively.

In Section D, we will derive an expression for $W_{\text {ext }}$ and solve Eqs. (65), (67). In Section E, we will derive a new expression for $W_{\text {ext }}$ in the nanoscopic regime, and solve Eqs. (62), (64).

\section{Efficiency of a heat engine according to macroscopic thermodynamics}

In this section, we study the efficiency of the setup detailed in Section A under the constraints of macroscopic thermodynamics, as described in Section B.1. This implies that the non-equilibrium free energy solely dictates whether $\rho_{\text {ColdW }}^{0} \rightarrow \rho_{\text {ColdW }}^{1}$ is possible. We find that in both cases of extracting perfect and near perfect work, 
(1) The maximum achievable efficiency is the Carnot efficiency.

(2) The Carnot efficiency can be achieved for any cold bath Hamiltonian.

(3) For any $\Delta C$, the maximum efficiency achievable for the particular value of $\Delta C$, is achieved iff the final state of the cold bath is thermal (according to a different temperature $T_{f}$ ).

(4) When the final state of the cold bath is thermal, the Carnot efficiency is achieved iff we take the limit corresponding to a quasi-static heat engine (Eq. (69)). Roughly speaking, this means that there is only infinitesimal change in the final temperature of the cold bath, compared to its original state.

This section can be summarized as follows: in Section D.1, we first apply the macroscopic law of thermodynamics, namely the fact that non-equilibrium free energy is non-increasing, to our heat engine setup. By making use of energy conservation, we can derive the amount of maximum extractable work as shown in Eq. (81). Next, in Section D.2 we show that when considering the extraction of perfect work, we show the points (1)-(4) as stated above. In Section D.3, we show that points (1)-(4) hold also when considering near perfect work.

The main results can be found in Theorem 1 and Lemma 6. One may think points (1)-(4) are obvious since it has long been known that the optimal achievable efficiency of a heat engine operating between two thermal baths is the Carnot efficiency, and that this efficiency can be achieved quasi-statically. The motivations for proving these results here are two-fold. Firstly, this is a rigorous and mathematical proof of optimality, while usually one encounters arguments such as reversibility, or that the heat engine must remain in thermal equilibrium at all times during the working of the heat engine. Secondly, we will find later on at the nano/quantum scale that the Carnot efficiency can be achieved but observation (2) does not hold anymore. For these reasons, it is worthwhile proving that one can actually achieve points (1)-(4) in this setting for any cold bath Hamiltonian according to macroscopic thermodynamics. From a practical point of view, many of the technical results proved here will be needed in the proofs of Section E, where we derive results involving a more refined set of generalized free energies, which describes thermodynamic transitions for nanoscale quantum systems.

\section{D.1 Maximum extractable work according to macroscopic law of thermodynamics}

Our first task is to find an expression for $W_{\text {ext }}$ in the macro regime. We do so by solving Eq. (49) for $W_{\text {ext }}$ such that

$$
\left\langle\hat{H}_{\text {ColdMW }}\right\rangle_{\rho_{\text {ColdMw }}^{1}}-\frac{1}{\beta_{h}} S\left(\rho_{\text {ColdMW }}^{1}\right) \leq\left\langle\hat{H}_{\text {ColdMW }}\right\rangle_{\rho_{\text {ColdMw }}^{0}}-\frac{1}{\beta_{h}} S\left(\rho_{\text {ColdMW }}^{0}\right) .
$$

The entropy is an additive quantity under tensor product, meaning that $S\left(\rho_{1} \otimes \rho_{2}\right)=S\left(\rho_{1}\right)+S\left(\rho_{2}\right)$ for any states $\rho_{1}, \rho_{2}$. Furthermore, since the joint Hamiltonian does not contain interaction terms, therefore the mean energy also depends only on the reduced states. In summary, both $S$ and $\langle\hat{H}\rangle$ are additive under a tensor product structure of $\rho_{\text {ColdMw }}^{0}$ and $\rho_{\text {ColdMw }}^{1}$ as described in Eqs. (38) and (39). This means one can rewrite Eq. (78) by expanding its terms,

$$
\begin{gathered}
\left\langle\hat{H}_{\text {Cold }}\right\rangle_{\rho_{\text {Cold }}^{1}}+\left\langle\hat{H}_{\mathrm{M}}\right\rangle_{\rho_{\mathrm{M}}^{1}}+\left\langle\hat{H}_{\mathrm{W}}\right\rangle_{\rho_{\mathrm{W}}^{1}}-\frac{1}{\beta_{h}}\left[S\left(\rho_{\text {Cold }}^{1}\right)+S\left(\rho_{\mathrm{M}}^{1}\right)+S\left(\rho_{\mathrm{W}}^{1}\right)\right] \leq \\
\left\langle\hat{H}_{\text {Cold }}\right\rangle_{\rho_{\text {Cold }}^{0}}+\left\langle\hat{H}_{\mathrm{M}}\right\rangle_{\rho_{\mathrm{M}}^{0}}+\left\langle\hat{H}_{\mathrm{W}}\right\rangle_{\rho_{\mathrm{W}}^{0}}-\frac{1}{\beta_{h}}\left[S\left(\rho_{\text {Cold }}^{0}\right)+S\left(\rho_{\mathrm{M}}^{0}\right)+S\left(\rho_{\mathrm{W}}^{0}\right)\right],
\end{gathered}
$$

Furthermore, note that $\rho_{\mathrm{M}}^{0}=\rho_{\mathrm{M}}^{1}$, and therefore $S\left(\rho_{\mathrm{M}}^{0}\right),\left\langle\hat{H}_{\mathrm{M}}\right\rangle_{\rho_{\mathrm{M}}^{0}}$ are common terms on both sides of Eq. (79) which can be cancelled out. Furthermore, by our construction of the battery in Eqs. (40)-(43), 
we have that $S\left(\rho_{\mathrm{W}}^{0}\right)=0, S\left(\rho_{\mathrm{W}}^{1}\right)=\Delta S=\mathrm{h}_{2}(\varepsilon)$ and $\left\langle\hat{H}_{\mathrm{W}}\right\rangle_{\rho_{\mathrm{W}}^{0}}=E_{j}^{\mathrm{W}}$ and $\left\langle\hat{H}_{\mathrm{W}}\right\rangle_{\rho_{\mathrm{W}}^{1}}=(1-\varepsilon) E_{k}^{W}+\varepsilon E_{j}^{W}$. Thus, Eq. (79) can be simplified to

$$
(1-\varepsilon) W_{\text {ext }}+\left\langle\hat{H}_{\text {Cold }}\right\rangle_{\rho_{\text {Cold }}^{1}}-\frac{1}{\beta_{h}} S\left(\rho_{\text {Cold }}^{1}\right) \leq\left\langle\hat{H}_{\text {Cold }}\right\rangle_{\rho_{\text {Cold }}^{0}}-\frac{1}{\beta_{h}} S\left(\rho_{\text {Cold }}^{0}\right)+\frac{1}{\beta_{h}} \mathrm{~h}_{2}(\varepsilon),
$$

where $W_{\text {ext }}$ has been defined in Eq. (43). In other words, $(1-\varepsilon) W_{\text {ext }} \leq F\left(\rho_{\text {Cold }}^{0}\right)-F\left(\rho_{\text {Cold }}^{1}\right)+\frac{1}{\beta_{h}} \mathrm{~h}_{2}(\varepsilon)$.

We can also express $W_{\text {ext }}$ with the relative entropy instead, by using Eq. (54). We can apply this identity to Eq. (80) whenever the initial and final states are diagonal in the energy eigenbasis. Note that the initial $\rho_{\text {Cold }}^{0}$ is a thermal state (of some temperature), and therefore diagonal in the energy eigenbasis. Since we start with a state $\tau_{\mathrm{Cold}}^{0} \otimes \rho_{\mathrm{W}}^{0}$ which is diagonal w.r.t. the Hamiltonian, and since catalytic thermal operations can never increase coherences between energy eigenstates (or in the macro setting, since we only demand mean energy conservation), we know that the final state $\rho_{\text {Cold }}^{1} \otimes \rho_{\mathrm{W}}^{1}$ is also diagonal in the energy eigenbasis. Therefore, Eq. (80) can be rewritten w.r.t. the relative entropies as follows

$$
(1-\varepsilon) W_{\text {ext }} \leq F\left(\rho_{\text {Cold }}^{0}\right)-F\left(\rho_{\text {Cold }}^{1}\right)+\frac{1}{\beta_{h}} \mathrm{~h}_{2}(\varepsilon)=\frac{1}{\beta_{h}}\left[D\left(\rho_{\text {Cold }}^{0} \| \tau_{\text {Cold }}^{h}\right)-D\left(\rho_{\text {Cold }}^{1} \| \tau_{\text {Cold }}^{h}\right)+\mathrm{h}_{2}(\varepsilon)\right] .
$$

\section{D.2 Maximum efficiency for perfect work is Carnot efficiency}

In this section, we want to find the maximum efficiency according to Eqs. (61), (65) and (67), for the case of $\varepsilon=0$ which implies $\mathrm{h}_{2}(\varepsilon)=0$. We do this by the following steps:

1. Evaluate $W_{\text {ext }}$. According to Eq. (81), we know that

$$
W_{\text {ext }}=F\left(\rho_{\text {Cold }}^{0}\right)-F\left(\rho_{\text {Cold }}^{1}\right)=\frac{1}{\beta_{h}}\left[D\left(\rho_{\text {Cold }}^{0} \| \tau_{\text {Cold }}^{h}\right)-D\left(\rho_{\text {Cold }}^{1} \| \tau_{\text {Cold }}^{h}\right)\right],
$$

where recall that we have defined $\tau_{\text {Cold }}^{h}$ previously as the thermal state of system Cold with temperature $T_{\text {Hot }}$. Note that here equality can be achieved because in macroscopic thermodynamics, satisfying the free energy constraint is a necessary and sufficient condition for the possibility of a state transformation. Note that since by construction the initial and final states of the battery are pure energy eigenstates, namely $\varepsilon=0$ and therefore

$$
W_{\text {ext }}=\Delta W
$$

2. Write inverse maximum efficiency as optimization problem. By substituting the simplified expression for efficiency derived in Eq. (77) into Eq. (67), we have

$$
\eta_{\max }^{-1}=\inf _{\rho_{\text {Cold }}^{1}}\left(\eta^{\text {mac }}\right)^{-1}=1+\inf _{\rho_{\text {Cold }}^{1}} \frac{\Delta C}{W_{\text {ext }}} .
$$

3. Maximize $W_{\text {ext }}$ given a fixed value of $\Delta C$. This is done in Lemma 1 , where we show that given a fixed $\Delta C$, the final cold bath state that maximizes $W_{\text {ext }}$ is uniquely a thermal state, corresponding to a certain inverse temperature $\beta^{\prime}$.

4. Show that 3) implies that efficiency is maximized by a thermal state of the cold bath. This is proven in Lemma 2. Therefore, this implies one only needs to optimize Eq. (84) over one variable, i.e. $\beta_{f}$, the final temperature of the cold bath.

5. Show that the efficiency is strictly increasing with $\beta_{f}$. This is done first by proving several identities, which are summarized in Corollary 1. Using these identities, we prove in Lemma 4 that the first derivative of efficiency w.r.t. $\beta_{f}$ is always positive over the range where $W_{\text {ext }}>0$. This leads us to conclude, in Theorem 1, that maximum efficiency is achieved in the limit $\beta_{f} \rightarrow \beta_{c}$, and evaluating the efficiency at this limit gives us the Carnot efficiency. 
Firstly, let us develop a technical Lemma 1, which concerns the unique solution towards maximizing $W_{\text {ext }}$ for a fixed $\Delta C$. By applying Lemma 1, we show in Lemma 2 that the maximal efficiency is achieved when $\rho_{\text {Cold }}^{1}$ is a thermal state. The reader can easily find similar proofs in [64].

Lemma 1. Given any Hamiltonian $\hat{H}_{\text {Cold }}$, a corresponding thermal state $\tau_{\text {Cold }}^{h}$ of some temperature $\beta_{h}$, and a fixed initial state $\rho_{\text {Cold }}^{0}$, consider the maximization over final states $\rho_{\text {Cold }}^{1}$,

$$
\max _{\rho_{\text {Cold }}^{1}} W_{\text {ext }}=\frac{1}{\beta_{h}}\left[D\left(\rho_{\text {Cold }}^{0} \| \tau_{\text {Cold }}^{h}\right)-\min _{\rho_{\text {Cold }}^{1}} D\left(\rho_{\text {Cold }}^{1} \| \tau_{\text {Cold }}^{h}\right)\right] .
$$

over all states $\rho_{\text {Cold }}^{1}$ which are diagonal in the energy eigenbasis, subject to the constraint that $\Delta C$ is a constant. Then the solution for $\rho_{\text {Cold }}^{1}$ is unique, and $\rho$ is a thermal state according to the Hamiltonian $\hat{H}_{\text {Cold }}$ at a certain temperature $\beta^{\prime}$.

Proof. Firstly, from Eq. (74) we see that the constraint $\Delta C$ being a constant, is the same as $\operatorname{tr}\left[\hat{H}_{\text {Cold }} \rho_{\text {Cold }}^{1}\right]$ being a constant. This is because they differ only by a constant term. On the other hand, from Eq. (75) and (83), we can see that $\max _{\rho_{\text {Cold }}^{1}} W_{\text {ext }}$ is equal to the R.H.S. of Eq. (85). Since $\rho_{\text {Cold }}^{1}$ and $\tau$ are both diagonal in the energy eigenbasis ( $\rho_{\text {Cold }}^{1}$ by the statement in the lemma, and $\tau$ by it being a thermal state), one can evaluate the relative entropy by using Eq. (51). Denote the eigenvalues of our variable $\rho_{\text {Cold }}^{1}$ to be $\left\{p_{i}\right\}_{i}$, and the eigenvalues of the thermal state $\tau$ to be $\left\{q_{i}\right\}_{i}$. We can then write the optimization problem as

$$
\begin{gathered}
\min _{\left\{p_{i}\right\}} \sum_{i} p_{i}\left(\ln p_{i}-\ln q_{i}\right) ; \quad \text { subject to } \sum_{i} p_{i} E_{i}=c \quad \text { constant, and } \sum_{i} p_{i}=1 . \\
\text { where } \quad q_{i}=\frac{e^{-\beta E_{i}}}{Z_{\beta}} ; \quad Z_{\beta}=\sum_{i} e^{-\beta E_{i}} .
\end{gathered}
$$

We can now employ techniques of Lagrange multipliers to solve this optimization. The constrained Lagrange equation is

$$
\begin{aligned}
L\left(\left\{p_{i}\right\}, \lambda\right) & =\sum_{i} p_{i}\left(\ln p_{i}-\ln q_{i}\right)+\lambda\left(\sum_{i} E_{i} p_{i}-c\right)+\mu\left(\sum_{i} p_{i}-1\right), \\
\frac{d L}{d p_{i}} & =\left(\ln p_{i}-\ln q_{i}+1+\lambda E_{i}+\mu\right)=0, \\
\frac{d L}{d \lambda} & =\sum_{i} E_{i} p_{i}-c=0 . \\
\frac{d L}{d \mu} & =\sum_{i} p_{i}-1=0 .
\end{aligned}
$$

We find that the normalized solution is

$$
p_{i}=\frac{e^{-\beta^{\prime} E_{i}}}{Z_{\beta^{\prime}}}, \quad Z_{\beta^{\prime}}=e^{(1+\mu)} Z_{\beta},
$$

and $p_{i}$ are probabilities corresponding to the Boltzmann distribution, according to inverse temperature $\beta^{\prime}=\beta+\lambda$. Depending on the mean energy constraint $c$ and normalization condition, one can solve for the Lagrange multipliers $\lambda$ and $\mu$. With this we conclude that the state $\rho$ which maximizes $D\left(\rho_{\text {Cold }}^{1} \| \tau\right)$ is a thermal state, where its temperature is such that the constraint on mean energy is satisfied.

Lemma 2. Consider the work extraction process described by the state transformation $\rho_{\text {ColdMw }}^{0} \rightarrow$ $\rho_{\text {ColdMW }}^{1}$, where $\rho_{\text {Cold }}^{0}, \rho_{\mathrm{W}}^{0}$ and $\rho_{\mathrm{W}}^{1}$ have been described in Section A. Denote $\mathcal{H}_{\text {Cold }}$ as the Hilbert space of the cold bath. Then the maximal efficiency in Eq. (84) is obtained for a final state of the cold bath $\rho_{\text {Cold }}^{1}$, which is thermal:

$$
\eta_{\max }^{-1}=1+\inf _{\rho_{\text {Cold }}^{1} \in \mathcal{S}_{\tau}} \frac{\Delta C}{W_{\text {ext }}}
$$


where $\mathcal{S}_{\tau}$ the set of all thermal states (for $\hat{H}_{\text {Cold }}$ with any temperature $T>0$ ) in $\mathcal{H}_{\text {Cold }}$. Furthermore, all non-thermal states do not achieve the maximum efficiency, i.e.

$$
\eta_{\max }^{-1}<1+\left.\frac{\Delta C}{W_{\text {ext }}}\right|_{\rho_{\text {Cold }}^{1}} \quad \text { for any } \rho_{\text {Cold }}^{1} \in \mathcal{S} \backslash \mathcal{S}_{\tau}
$$

where $\mathcal{S}$ is the space of all quantum states in $\mathcal{H}_{\text {Cold }}$

Proof. First of all, note that without loss of generality we can always consider only diagonal states, as explained in the paragraph before Eq. (81) that catalytic thermal operations do not increase coherences between energy eigenstates. We begin by substituting Eqs. (74) and (82) into Eq. (84), and finding

$$
\begin{aligned}
\eta_{\max }^{-1} & =1+\inf _{\rho_{\text {Cold }}^{1}} \frac{\Delta C}{W_{\text {ext }}} \\
& =1+\inf _{\rho_{\text {Cold }}^{1}} \frac{\beta_{h} \Delta C}{D_{1}\left(\tau_{\text {Cold }}^{c} \| \tau_{\text {Cold }}^{h}\right)-D_{1}\left(\rho_{\text {Cold }}^{1} \| \tau_{\text {Cold }}^{h}\right)} \\
& =1+\beta_{h}\left[\sup _{\rho_{\text {Cold }}^{1}} \frac{D_{1}\left(\tau_{\text {Cold }}^{c} \| \tau_{\text {Cold }}^{h}\right)-D_{1}\left(\rho_{\text {Cold }}^{1} \| \tau_{\text {Cold }}^{h}\right)}{\operatorname{tr}\left(\hat{H}_{\text {Cold }} \rho_{\text {Cold }}^{1}\right)-\operatorname{tr}\left(\hat{H}_{\text {Cold }} \tau_{\text {Cold }}^{c}\right)}\right]^{-1} .
\end{aligned}
$$

In the last line of Eq. (95), we see that only two terms depend on the maximization variable $\rho_{\text {Cold }}^{1}$. This means we can perform the maximization in two steps:

$$
\sup _{\rho_{\text {Cold }}^{1}} \frac{D_{1}\left(\tau_{\text {Cold }}^{c} \| \tau_{\text {Cold }}^{h}\right)-D_{1}\left(\rho_{\text {Cold }}^{1} \| \tau_{\text {Cold }}^{h}\right)}{\operatorname{tr}\left(\hat{H}_{\text {Cold }} \rho_{\text {Cold }}^{1}\right)-\operatorname{tr}\left(\hat{H}_{\text {Cold }} \tau_{\text {Cold }}^{c}\right)}=\sup _{A>0} \frac{D_{1}\left(\tau_{\text {Cold }}^{c} \| \tau_{\text {Cold }}^{h}\right)-B(A)}{A}
$$

where $B(A)$ is the optimal value of a separate minimization problem:

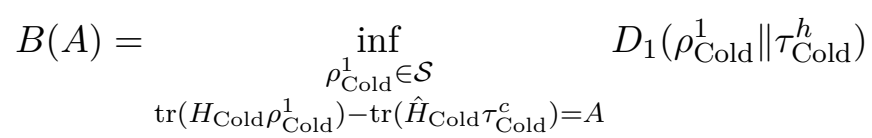

From Lemma 1, we know that the solution of the sub-minimization problem in Eq. (97) has a unique form, namely $\rho_{\text {Cold }}^{1}=\tau_{\text {Cold }}^{f}$ is a thermal state of some temperature $\beta_{f}$. Therefore, Eq. (96) can be simplified to

$$
\sup _{\rho_{\text {Cold }}^{1}} \frac{D_{1}\left(\tau_{\text {Cold }}^{c} \| \tau_{\text {Cold }}^{h}\right)-D_{1}\left(\rho_{\text {Cold }}^{1} \| \tau_{\text {Cold }}^{h}\right)}{\operatorname{tr}\left(\hat{H}_{\text {Cold }} \rho_{\text {Cold }}^{1}\right)-\operatorname{tr}\left(\hat{H}_{\text {Cold }} \tau_{\text {Cold }}^{c}\right)}=\sup _{\beta_{f}} \frac{D_{1}\left(\tau_{\text {Cold }}^{c} \| \tau_{\text {Cold }}^{h}\right)-D_{1}\left(\tau_{\text {Cold }}^{f} \| \tau_{\text {Cold }}^{h}\right)}{\operatorname{tr}\left(\hat{H}_{\text {Cold }} \tau_{\text {Cold }}^{f}\right)-\operatorname{tr}\left(\hat{H}_{\text {Cold }} \tau_{\text {Cold }}^{c}\right)} .
$$

Whats more, for every constant $A$, the function

$$
f(x)=\left(1+\beta_{h}\left[\frac{D_{1}\left(\tau_{\text {Cold }}^{c} \| \tau_{\text {Cold }}^{h}\right)-x}{A}\right]^{-1}\right)^{-1}
$$

is bijective in $x \in \mathbb{R}$ and thus due to the uniqueness of the sub-minimization problem in Eq. (97), we conclude that for all non-thermal states $\rho_{\text {Cold }}^{1}$, the corresponding efficiency will be strictly less than that of Eq. (95). Thus from Eq. (98) and (95) we conclude the lemma.

After establishing Lemma 2, we can continue to solve the optimization problem in Eq. (84) by only looking at final states which are thermal (according to some final temperature $\beta_{f}$ which we optimize over). In the next Lemma 3 and Corollary 1, we derive some useful and interesting identities. These identities concern quantities such as the derivatives of mean energy and entropy of the thermal state (with respect to inverse temperature), and relates them to the variance of energy. The reader can find similar proofs in any standard thermodynamic textbook (For example in Sections 6.5, 6. of [60]), but we derive them here for completeness. 
Lemma 3. For any cold bath Hamiltonian $\hat{H}_{\text {Cold }}$, consider the thermal state $\tau_{\beta}=\frac{1}{Z_{\beta}} e^{-\beta \hat{H}_{\text {Cold }}}$ with inverse temperature $\beta$. Define $\left\langle\hat{H}_{\text {Cold }}\right\rangle_{\beta}=\operatorname{tr}\left(\hat{H}_{\text {Cold }} \tau_{\beta}\right)$, and $S(\beta)=-\tau_{\beta} \ln \tau_{\beta}$ to be the mean energy and entropy of $\tau_{\beta}$. Then the following identities hold:

$$
\begin{aligned}
\frac{d\left\langle\hat{H}_{\text {Cold }}\right\rangle_{\beta}}{d \beta} & =-\operatorname{var}\left(\hat{H}_{\text {Cold }}\right)_{\beta} \\
\frac{d S(\beta)}{d \beta} & =-\beta \cdot \operatorname{var}\left(\hat{H}_{\text {Cold }}\right)_{\beta},
\end{aligned}
$$

where $\operatorname{var}\left(\hat{H}_{\text {Cold }}\right)_{\beta}=\left\langle\hat{H}_{\text {Cold }}^{2}\right\rangle_{\beta}-\left\langle\hat{H}_{\text {Cold }}\right\rangle_{\beta}^{2}$ is the variance of energy for $\tau_{\beta}$.

Proof. Intuitively we know that the expectation value of energy increases as temperature increases (or as the inverse temperature decreases). More precisely, consider the probabilities of $\tau_{\beta}$ for each energy level of the Hamiltonian $E_{i}$,

$$
\begin{aligned}
p_{i} & =\frac{e^{-\beta E_{i}}}{Z_{\beta}}, \quad \text { where } Z_{\beta}=\sum_{i} e^{-\beta E_{i}} \\
\frac{d p_{i}}{d \beta} & =\frac{1}{Z_{\beta}^{2}}\left[-E_{i} e^{-\beta E_{i}} \cdot Z_{\beta}-\frac{d Z_{\beta}}{d \beta} \cdot e^{-\beta E_{i}}\right]=-p_{i} E_{i}-\frac{1}{Z_{\beta}} \frac{d Z_{\beta}}{d \beta} p_{i}=-p_{i} E_{i}+p_{i}\left\langle\hat{H}_{\text {Cold }}\right\rangle_{\beta} .
\end{aligned}
$$

The last equality holds because of the following identity:

$$
\frac{-1}{Z} \frac{d Z}{d \beta}=\frac{-1}{Z} \sum_{i}\left(-E_{i}\right) e^{-\beta E_{i}}=\sum_{i} p_{i} E_{i}=\left\langle\hat{H}_{\text {Cold }}\right\rangle_{\beta} .
$$

Therefore, we have

$$
\begin{aligned}
\frac{d\left\langle\hat{H}_{\text {Cold }}\right\rangle_{\beta}}{d \beta} & =\sum_{i} \frac{d\left\langle\hat{H}_{\text {Cold }}\right\rangle_{\beta}}{d p_{i}} \frac{d p_{i}}{d \beta}=\sum_{i} E_{i} \cdot\left[-p_{i} E_{i}+p_{i}\left\langle\hat{H}_{\text {Cold }}\right\rangle_{\beta}\right] \\
& =-\left\langle\hat{H}_{\text {Cold }}^{2}\right\rangle_{\beta}+\left\langle\hat{H}_{\text {Cold }}\right\rangle_{\beta}^{2}=-\operatorname{var}\left(\hat{H}_{\text {Cold }}\right)_{\beta} .
\end{aligned}
$$

On the other hand, similarly, one can prove the second identity by writing down the expression of entropy for the thermal state,

$$
S(\beta)=-\sum_{i} \frac{e^{-\beta E_{i}}}{Z_{\beta}} \ln \frac{e^{-\beta E_{i}}}{Z_{\beta}}=\sum_{i} \beta E_{i} \frac{e^{-\beta E_{i}}}{Z_{\beta}}+\ln Z_{\beta} \sum_{i} \frac{e^{-\beta E_{i}}}{Z_{\beta}}=\beta\left\langle\hat{H}_{\text {Cold }}\right\rangle_{\beta}+\ln Z_{\beta} .
$$

Therefore, the derivative of $S(\beta)$ w.r.t. $\beta$ is

$$
\frac{d S\left(\tau_{\beta}\right)}{d \beta}=\left\langle\hat{H}_{\text {Cold }}\right\rangle_{\beta}+\beta \frac{d\left\langle\hat{H}_{\text {Cold }}\right\rangle_{\beta}}{d \beta}+\frac{1}{Z_{\beta}} \frac{d Z_{\beta}}{d \beta}=\beta \cdot \frac{d\left\langle\hat{H}_{\text {Cold }}\right\rangle_{\beta}}{d \beta}=-\beta \cdot \operatorname{var}\left(\hat{H}_{\text {Cold }}\right)_{\beta} .
$$

By using Lemma 3 in a special case, we obtain the following corollary:

Corollary 1. Given any Hamiltonian $\hat{H}_{\text {Cold }}$, consider the quantities

$$
\Delta C\left(\beta_{f}\right)=\operatorname{tr}\left(\hat{H}_{\text {Cold }} \tau_{\beta_{f}}\right)-\operatorname{tr}\left(\hat{H}_{\text {Cold }} \tau_{\beta_{c}}\right)=\left\langle\hat{H}_{\text {Cold }}\right\rangle_{\beta_{f}}-\left\langle\hat{H}_{\text {Cold }}\right\rangle_{\beta_{c}}
$$

and

$$
W_{\text {ext }}\left(\beta_{f}\right)=F\left(\tau_{\beta_{c}}\right)-F\left(\tau_{\beta_{f}}\right)=\frac{1}{\beta_{h}}\left[D\left(\tau_{\beta_{c}} \| \tau_{\beta_{h}}\right)-D\left(\tau_{\beta_{f}} \| \tau_{\beta_{h}}\right)\right],
$$

where $\tau_{\beta}$ corresponds to the thermal state defined by $\hat{H}_{\text {Cold }}$ at inverse temperature $\beta$. Then

$$
\begin{aligned}
& \frac{d \Delta C\left(\beta_{f}\right)}{d \beta_{f}}=-\operatorname{var}\left(\hat{H}_{\text {Cold }}\right)_{\beta_{f}} \\
& \frac{d W_{\text {ext }}\left(\beta_{f}\right)}{d \beta_{f}}=\frac{\beta_{h}-\beta_{f}}{\beta_{h}} \operatorname{var}\left(\hat{H}_{\text {Cold }}\right)_{\beta_{f}} .
\end{aligned}
$$


Proof. For $\Delta C\left(\beta_{f}\right)$, it is straightforward from Lemma 3 that

$$
\frac{d \Delta C\left(\beta_{f}\right)}{d \beta_{f}}=\frac{d\left\langle\hat{H}_{\mathrm{Cold}}\right\rangle_{\beta_{f}}}{d \beta_{f}}=-\operatorname{var}\left(\hat{H}_{\mathrm{Cold}}\right)_{\beta_{f}} .
$$

On the other hand, $\Delta W\left(\beta_{f}\right)$ can be simplified by substituting Eq. (54) into Eq. (109),

$$
W_{\text {ext }}\left(\beta_{f}\right)=F\left(\tau_{\beta_{c}}\right)-F\left(\tau_{\beta_{f}}\right)=\left\langle\hat{H}_{\text {Cold }}\right\rangle_{\beta_{c}}-\left\langle\hat{H}_{\text {Cold }}\right\rangle_{\beta_{f}}-\frac{1}{\beta_{h}}\left[S\left(\tau_{\beta_{c}}\right)-S\left(\tau_{\beta_{f}}\right)\right] .
$$

With this, we can evaluate the derivative

$$
\begin{aligned}
\frac{d W_{\text {ext }}\left(\beta_{f}\right)}{d \beta_{f}} & =-\frac{d\left\langle\hat{H}_{\text {Cold }}\right\rangle_{\beta_{f}}}{d \beta_{f}}+\frac{1}{\beta_{h}} \frac{d S\left(\tau_{\beta_{f}}\right)}{d \beta_{f}} \\
& =\operatorname{var}\left(\hat{H}_{\text {Cold }}\right)_{\beta_{f}}-\frac{\beta_{f}}{\beta_{h}} \operatorname{var}\left(\hat{H}_{\text {Cold }}\right)_{\beta_{f}} \\
& =\frac{\beta_{h}-\beta_{f}}{\beta_{h}} \operatorname{var}\left(\hat{H}_{\text {Cold }}\right)_{\beta_{f}} .
\end{aligned}
$$

The second equality is obtained by Lemma 3 for $\frac{d\left\langle\hat{H}_{\text {Cold }}\right\rangle_{\beta_{f}}}{d \beta_{f}}$, and the third by grouping common factors together.

In the next step, by using Corollary 1, we show that when the final state of the cold bath is thermal, the optimal efficiency is achieved only in the quasi-static limit, i.e. in the limit $\beta_{f} \rightarrow \beta_{c}$ when the efficiency is optimised over all final thermal states of the cold bath.

Lemma 4. Evaluate the efficiency expressed in Eq. (77) for the situation where the final state of the cold bath is a thermal state at inverse temperature $\beta_{f}$ :

$$
\eta\left(\beta_{f}\right)=\frac{W_{\mathrm{ext}}\left(\beta_{f}\right)}{\Delta C\left(\beta_{f}\right)+W_{\mathrm{ext}}\left(\beta_{f}\right)} .
$$

Then for all $\beta_{f}<\beta_{c}, \frac{d \eta\left(\beta_{f}\right)}{d \beta_{f}}>0$.

Proof. To prove this, we show that $\frac{d \eta^{-1}}{d \beta_{f}}<0$, where $\eta^{-1}=1+\frac{\Delta C}{W_{\text {ext }}}$. Evaluating the derivative of $\eta^{-1}$ w.r.t. $\beta_{f}$, we obtain

$$
\begin{aligned}
\frac{d \eta^{-1}}{d \beta_{f}} & =\frac{1}{W_{\mathrm{ext}}^{2}} \cdot\left[\frac{d \Delta C\left(\beta_{f}\right)}{d \beta_{f}} W_{\mathrm{ext}}-\frac{d W_{\mathrm{ext}}\left(\beta_{f}\right)}{d \beta_{f}} \Delta C\right] \\
& =\frac{\operatorname{var}\left(\hat{H}_{\mathrm{Cold}}\right) \beta_{f}}{W_{\mathrm{ext}}^{2}} \cdot\left[-W_{\mathrm{ext}}-\frac{\beta_{h}-\beta_{f}}{\beta_{h}} \Delta C\right] \\
& =\frac{\operatorname{var}\left(\hat{H}_{\mathrm{Cold}}\right) \beta_{f}}{W_{\mathrm{ext}}^{2}} \cdot\left[\Delta C+\frac{1}{\beta_{h}}\left[S\left(\tau_{\beta_{c}}\right)-S\left(\tau_{\beta_{f}}\right)\right]-\frac{\beta_{h}-\beta_{f}}{\beta_{h}} \Delta C\right] \\
& =\frac{\operatorname{var}\left(\hat{H}_{\mathrm{Cold}}\right)_{\beta_{f}}}{W_{\mathrm{ext}}^{2}} \frac{\beta_{f}}{\beta_{h}} \cdot\left[\Delta C-\frac{1}{\beta_{f}}\left[S\left(\tau_{\beta_{f}}\right)-S\left(\tau_{\beta_{c}}\right)\right]\right]
\end{aligned}
$$

The first equality is obtained by invoking the chain rule of differentiation. The second equality is obtained by substituting $\frac{d W_{\text {ext }}}{d \beta_{f}}, \frac{d \Delta C}{d \beta_{f}}$, as evaluated earlier in Corollary 1 . The third equality is obtained by expressing $W_{\text {ext }}$ according to Eq. (113), plus recognizing that $\left\langle\hat{H}_{\text {Cold }}\right\rangle_{\tau_{\beta_{f}}}-\left\langle\hat{H}_{\text {Cold }}\right\rangle_{\tau_{\beta_{c}}}=\Delta C$. The last inequality is obtained, simply by taking out a common term $\beta_{f} / \beta_{h}$. We then make the following 
observations:

1) The factor

$$
\frac{\beta_{f}}{\beta_{h} W_{\mathrm{ext}}^{2}}>0
$$

2) The variance of energy for any positive temperature

$$
\operatorname{var}\left(\hat{H}_{\text {Cold }}\right)_{\beta_{f}}>0
$$

3) and the last term $\Delta C-\frac{1}{\beta_{f}}\left[S\left(\tau_{\beta_{f}}\right)-S\left(\tau_{\beta_{c}}\right)\right]$ can be written as $F\left(\tau_{\beta_{f}}\right)-F\left(\tau_{\beta_{c}}\right)$, where $F$ is the free energy of a system w.r.t. a bath with inverse temperature $\beta_{f}$. But then, since $\tau_{\beta_{f}}$ is the thermal state with the same inverse temperature, this means that $\tau_{\beta_{f}}$ is the unique state that minimizes free energy. Therefore, $F\left(\tau_{\beta_{c}}\right)-F\left(\tau_{\beta_{f}}\right)>0$ for any $\tau_{\beta_{c}}$.

From Lemma 2 and Lemma 4, we conclude that the maximization of efficiency for any Hamiltonian $\hat{H}$ happens for a final state which is thermal, and the greater its inverse temperature $\beta_{f}$, the higher efficiency is. With these lemmas we can now prove the main result of this section (Theorem 1).

In the next theorem, we evaluate the efficiency at the limit $\beta_{f} \rightarrow \beta_{c}^{-}$, and show that it corresponds to the Carnot efficiency.

Theorem 1 (Carnot Efficiency). Consider all heat engines which extract perfect work (see Definition 4). Then according to the macroscopic second law of thermodynamics, the maximum achievable efficiency (see Eq. (67)) is the Carnot efficiency

$$
\eta_{\max }=1-\frac{\beta_{h}}{\beta_{c}} .
$$

It can be obtained for all cold bath Hamiltonians $\hat{H}_{\text {Cold }}$, but when the final state of the cold bath $\rho_{\text {Cold }}^{1}$ is thermal, then only for quasi-static heat engines (as defined in Def. 6 and Eq. (69) for quasi-static maximum efficiency). In this quasi-static limit, an infinitesimal amount of work is extracted.

Proof. From Eq. (67), we have an expression for the optimal efficiency in terms of a maximization over final cold bath states $\rho_{\text {Cold }}^{1} \in \mathcal{S}$. By Lemma 2, we know that the optimal solution is obtained only for thermal states. Subsequently, by Lemma 4 , it is shown that when the final cold bath is of temperature $\beta_{f}$, the corresponding efficiency is strictly increasing w.r.t. $\beta_{f}$. Also note that since by definition $W_{\text {ext }}>0$, this implies that $\beta_{f}<\beta_{c}$. Intuitively, this is because heat cannot flow from a cold to hot system without any work input. One can also see this mathematically, by showing that for any $\beta \geq \beta_{h}$,

$$
\frac{d F\left(\tau_{\beta}\right)}{d \beta}=\frac{d}{d \beta}\left[\left\langle\hat{H}_{\text {Cold }}\right\rangle_{\beta}-\frac{1}{\beta_{h}} S(\beta)\right]=\left(\frac{\beta}{\beta_{h}}-1\right) \operatorname{var}\left(\hat{H}_{\text {Cold }}\right)_{\beta} \geq 0 .
$$

This implies that if $\beta_{f} \geq \beta_{c} \geq \beta_{h}$, then $F\left(\beta_{f}\right) \geq F\left(\beta_{c}\right)$, and according to Eq. (109) $W_{\text {ext }} \leq 0$. Therefore, when the final state of the cold bath $\rho_{\text {Cold }}^{1}$ is thermal, the optimal efficiency must be achieved only when its inverse temperature $\beta_{f}$ approaches $\beta_{c}$ from below. Let $\beta_{f}=\beta_{c}-g$, where $g>0$. Then we have

$$
\eta_{\max }^{-1}=\lim _{g \rightarrow 0^{+}}\left(\eta^{\mathrm{mac}}\right)^{-1}\left(\beta_{c}-g\right), \quad\left(\eta^{\mathrm{mac}}\right)^{-1}\left(\beta_{c}-g\right)=1+\left.\frac{\Delta C}{W_{\mathrm{ext}}}\right|_{\rho_{\mathrm{Cold}}^{1}=\tau_{\left(\beta_{c}-g\right)}} .
$$

Since as $g \rightarrow 0^{+}$, both the numerator and denominator vanish, we can evaluate this limit by first applying L'Hôspital rule, the chain rule for derivatives (for any function $F, \frac{d F}{d g}=-\frac{d F}{d \beta_{f}}$ ), and then Corollary 1 to obtain

$$
\lim _{g \rightarrow 0^{+}} \frac{\Delta C}{W_{\mathrm{ext}}}=\lim _{g \rightarrow 0^{+}} \frac{\frac{d \Delta C}{d g}}{\frac{d W_{\mathrm{ext}}}{d g}}=\lim _{\beta_{f} \rightarrow \beta_{c}^{-}} \frac{\frac{d \Delta C}{d \beta_{f}}}{\frac{d W_{\mathrm{ext}}}{d \beta_{f}}}=\frac{\beta_{h}}{\beta_{c}-\beta_{h}} .
$$


This implies that

$$
\eta_{\max }^{-1}=\lim _{g \rightarrow 0^{+}}\left(\eta^{\mathrm{mac}}\right)^{-1}\left(\beta_{c}-g\right)=1+\frac{\beta_{h}}{\beta_{c}-\beta_{h}}=\frac{\beta_{c}}{\beta_{c}-\beta_{h}}
$$

and hence $\eta_{\max }=1-\frac{\beta_{h}}{\beta_{c}}$.

\section{D.3 Maximum efficiency for near perfect work is still Carnot efficiency}

In this section, we show that even while allowing a non-zero failure probability $\varepsilon>0$ in the near perfect work scenario, the maximum achievable efficiency is still the Carnot efficiency. It is worth noting that this result is also important later, as an upper bound to maximum efficiency in the nanoscopic regime. We first prove it in Lemma 5 for the case where the final state of the battery is fixed as in Eq. (42). Then later, we show in Lemma 6 that Carnot efficiency is still the maximum, even if we allow a more general final battery state. Before we present the proof, it is useful for the reader to recall the definition of near perfect work (Def. 5) and quasi-static heat engines (Def. 6).

Lemma 5. Consider all heat engines which extract near perfect work (see Def. 5). Then according to the macroscopic second law of thermodyanmics, the maximum efficiency of a heat engine, $\eta_{\max }$ is the Carnot efficiency

$$
\eta_{\max }=\sup _{\rho_{\text {Cold }}^{1} \in \mathcal{S}} \eta^{\text {mac }}\left(\rho_{\text {Cold }}^{1}\right)=1-\frac{\beta_{h}}{\beta_{c}},
$$

and the supremum is achieved for quasi-static heat engines (see Def. (6) and Eq. (69)).

Proof. The ideas in this proof are very similar to that of Section D.2, and the main complication comes from proving that even if we allow $\varepsilon>0$, as long as $\Delta S / W_{\text {ext }}$ is arbitrarily small, the maximum efficiency cannot surpass the Carnot efficiency.

Let us begin by establishing the relevant quantities for near perfect work extraction. The amount of work extractable from the heat engine, when we have a probability of failure, according to the standard free energy can be obtained by solving Eq. (81). We thus have that the maximum $W_{\text {ext }}$ is

$$
W_{\mathrm{ext}}=\beta_{h}^{-1}(1-\varepsilon)^{-1}\left[D\left(\tau_{\beta_{c}} \| \tau_{\beta_{h}}\right)-D\left(\rho_{\text {Cold }}^{1} \| \tau_{\beta_{h}}\right)+\Delta S\right],
$$

where $\Delta S$ is defined in Eq. (45).

Before we continue with the analysis, we will note a trivial consequence of Eq. (126). Condition 1) in Def 5 implies that $(1-\varepsilon)^{-1}$ is upper bounded. The terms in square brackets in Eq. (126) are also clearly upper bounded for finite $\beta_{c}, \beta_{h}$. Hence $W_{\text {ext }}$ is bounded from above. $\Delta S$ is solely a function of $\varepsilon$ and only approaches zero in the limits $\varepsilon \rightarrow 0^{+}, \varepsilon \rightarrow 1^{-}$; and $\varepsilon \rightarrow 1^{-}$is forbidden by 1) in Def 5 . Thus if 1) and 2) in Def 5 are satisfied,

$$
\lim _{\varepsilon \rightarrow 0^{+}} \frac{\Delta S}{W_{\text {ext }}}=0
$$

In turn, if Eq. (127) is satisfied, then we have near perfect work by Def. 5. Thus Eq. (127) is satisfied iff we have near perfect work. We will use this result later in the proof.

Extracting a positive amount of near perfect work implies that we can rule out all states $\rho_{\text {Cold }}^{1}$ such that $D\left(\tau_{\beta_{c}} \| \tau_{\beta_{h}}\right) \leq D\left(\rho_{\text {Cold }}^{1} \| \tau_{\beta_{h}}\right)$ from the analysis. This can be proven by contradiction: if $D\left(\tau_{\beta_{c}} \| \tau_{\beta_{h}}\right) \leq D\left(\rho_{\text {Cold }}^{1} \| \tau_{\beta_{h}}\right)$, then from Eq. (126) $\beta_{h} W_{\text {ext }} \leq \Delta S /(1-\varepsilon)$ and together with 2) in Def 5 this would imply

$$
0<\beta_{h}(1-\varepsilon) \leq \frac{\Delta S}{W_{\text {ext }}}<p
$$

However, since from 1) Def. 5 we have $\varepsilon \leq l$, Eq. (128) cannot be satisfied for all $p>0$, leading to a contradiction. 
From Eq. (77) we have

$$
\eta_{\max }^{-1}=1-\varepsilon+\inf _{\rho_{\text {Cold }}^{1} \in \mathcal{S}} \frac{\Delta C}{W_{\text {ext }}}=(1-\varepsilon) \cdot\left[1+\frac{\beta_{h} \Delta C}{D\left(\tau_{\beta_{c}} \| \tau_{\beta_{h}}\right)-D\left(\rho_{\text {Cold }}^{1} \| \tau_{\beta_{h}}\right)+\Delta S}\right],
$$

where $\Delta C=\Delta C\left(\rho_{\text {Cold }}^{1}\right)$ and is defined in Eq. (74).

Firstly, let us show that with a similar analysis as shown in Lemma 2, the maximum efficiency occurs when $\rho_{\text {Cold }}^{1}$ is a thermal state. From Eq. (129), we have

$$
\begin{aligned}
\eta_{\max }^{-1} & =(1-\varepsilon)\left[1+\beta_{h} \inf _{\rho_{\text {Cold }}^{1} \in \mathcal{S}} \frac{\Delta C}{D\left(\tau_{\beta_{c}} \| \tau_{\beta_{h}}\right)-D\left(\rho_{\text {Cold }}^{1} \| \tau_{\beta_{h}}\right)+\Delta S}\right] \\
& =(1-\varepsilon)\left[1+\beta_{h} \inf _{A>0} \frac{A}{D\left(\tau_{\beta_{c}} \| \tau_{\beta_{h}}\right)-B(A)+\Delta S}\right]
\end{aligned}
$$

where

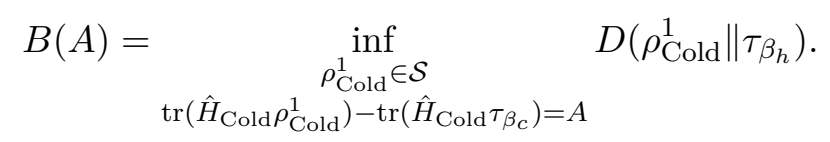

We can split this minimization problem to Eqs. (131) and (132) because $D\left(\tau_{\beta_{c}} \| \tau_{\beta_{h}}\right)$ and $\Delta S$ do not depend on the variable $\rho_{\text {Cold }}^{1}$. Furthermore, when $\rho_{\text {Cold }}^{1}$ is a thermal state of inverse temperature $\beta_{f}$, we have seen in the beginning of the proof in Theorem 1 that for $W_{\text {ext }}>0, \beta_{f}<\beta_{c}$. This implies that the variable $A=\Delta C=\operatorname{tr}\left(\hat{H}_{\text {Cold }} \tau_{\beta_{f}}\right)-\operatorname{tr}\left(\hat{H}_{\text {Cold }} \tau_{\beta_{c}}\right)>0$.

By Lemma 1, for any fixed $A>0$ we conclude that the infimum in Eq. (132) is achieved uniquely when $\rho_{\text {Cold }}^{1}$ is a thermal state. Therefore, our optimization problem is simplified to optimization over final temperatures $\beta_{f}$ (or $g=\beta_{c}-\beta_{f}$ ),

$$
\eta_{\max }^{-1}=(1-\varepsilon) \cdot\left[1+\beta_{h} \inf _{\substack{\beta_{f} \\ \Delta C>0}} \frac{\Delta C}{D\left(\tau_{\beta_{c}} \| \tau_{\beta_{h}}\right)-D\left(\tau_{\beta_{f}} \| \tau_{\beta_{h}}\right)+\Delta S}\right]
$$

Consider cases of $\beta_{f}$, where $D\left(\tau_{\beta_{c}} \| \tau_{\beta_{h}}\right)-D\left(\tau_{\beta_{f}} \| \tau_{\beta_{h}}\right)$ is non-vanishing (finite), i.e. which are not quasi-static. Note that this always corresponds to extracting near perfect work, since when $\varepsilon \rightarrow 0^{+}$, we have $\varepsilon, \Delta S \rightarrow 0$ and these contributions disappear from Eq. (133). However, by Lemma 2 we also know that the infimum over $\beta_{f}$ occurs uniquely at the quasi-static limit, when $g \rightarrow 0^{+}$.

What remains, is then to consider the quasi-static heat engine, namely the limit $g \rightarrow 0^{+}$. Extracting near perfect work in this case corresponds to requiring that $\lim _{g \rightarrow 0^{+}} \frac{\Delta S}{W_{\text {ext }}}=0$, where $\varepsilon=\varepsilon(g)$ and $\lim _{g \rightarrow 0^{+}} \varepsilon(g)=0$. Equivalently

$$
\lim _{g \rightarrow 0^{+}} \frac{W_{\text {ext }}}{\Delta S}=\infty
$$

Substituting Eq. (126) into Eq. (134),

$$
\lim _{g \rightarrow 0^{+}}(1-\varepsilon(g))^{-1}\left[1+\frac{D\left(\tau_{\beta_{c}} \| \tau_{\beta_{h}}\right)-D\left(\tau_{\beta_{f}} \| \tau_{\beta_{h}}\right)}{\Delta S}\right]=\infty
$$

which implies that $\lim _{g \rightarrow 0^{+}} \frac{D\left(\tau_{\beta_{c}} \| \tau_{\beta_{h}}\right)-D\left(\tau_{\beta_{f}} \| \tau_{\beta_{h}}\right)}{\Delta S}=\infty$, or equivalently,

$$
\lim _{\varepsilon \rightarrow 0^{+}} \lim _{g \rightarrow 0^{+}} \frac{\Delta S}{D\left(\tau_{\beta_{c}} \| \tau_{\beta_{h}}\right)-D\left(\tau_{\beta_{f}} \| \tau_{\beta_{h}}\right)}=0
$$


Finally, we evaluate the inverse efficiency at the quasi-static limit,

$$
\begin{aligned}
\eta^{-1} & =\lim _{g \rightarrow 0^{+}}(1-\varepsilon(g)) \cdot\left[1+\beta_{h} \frac{\Delta C}{D\left(\tau_{\beta_{c}} \| \tau_{\beta_{h}}\right)-D\left(\tau_{\beta_{f}} \| \tau_{\beta_{h}}\right)+\Delta S}\right] \\
& =1+\beta_{h} \lim _{g \rightarrow 0^{+}} \frac{\Delta C}{D\left(\tau_{\beta_{c}} \| \tau_{\beta_{h}}\right)-D\left(\tau_{\beta_{f}} \| \tau_{\beta_{h}}\right)+\Delta S} \\
& =1+\beta_{h} \lim _{g \rightarrow 0^{+}} \frac{\Delta C}{\left[D\left(\tau_{\beta_{c}} \| \tau_{\beta_{h}}\right)-D\left(\tau_{\beta_{f}} \| \tau_{\beta_{h}}\right)\right]} \cdot\left(1+\frac{\Delta S}{D\left(\tau_{\beta_{c}} \| \tau_{\beta_{h}}\right)-D\left(\tau_{\beta_{f}} \| \tau_{\beta_{h}}\right)}\right)^{-1} \\
& =1+\beta_{h} \lim _{g \rightarrow 0^{+}} \frac{d \Delta C\left(\tau_{\beta_{f}}\right) / d g}{d D\left(\tau_{\beta_{f}} \| \tau_{\beta_{h}}\right) / d g} \\
& =1-\frac{\beta_{h}}{\beta_{h}-\beta_{c}},
\end{aligned}
$$

where from Eq. (139) to (140), we make use of Eq. (136) : the second term within the limit is simply 1, and the first term depends only on $g$, which we can obtain Eq. (140) by invoking the L'Hôspital rule. The last equality in Eq. (141) follows directly from the identities we derived for $\frac{d W_{\text {ext }}}{d \beta_{f}}$ and $\frac{d \Delta C}{d \beta_{f}}$ in Corollary 1,

$$
\begin{aligned}
\frac{d \Delta C}{d g} & =-\frac{d \Delta C}{d \beta_{f}}=-\operatorname{var}\left(\hat{H}_{\text {Cold }}\right)_{\beta_{f}} \\
\frac{d D\left(\tau_{\beta_{f}} \| \tau_{\beta_{h}}\right)}{d g} & =-\frac{d D\left(\tau_{\beta_{f}} \| \tau_{\beta_{h}}\right)}{d \beta_{f}}=\beta_{h} \frac{d W_{\text {ext }}}{d \beta_{f}}=\left(\beta_{h}-\beta_{f}\right) \operatorname{var}\left(\hat{H}_{\text {Cold }}\right)_{\beta_{f}},
\end{aligned}
$$

while in the limit $g \rightarrow 0, \beta_{f}=\beta_{c}$.

Finally, we now see that the quasi-static efficiency is

$$
\eta=\left(\frac{\beta_{h}-\beta_{c}-\beta_{h}}{\beta_{h}-\beta_{c}}\right)^{-1}=\frac{\beta_{c}-\beta_{h}}{\beta_{c}}=1-\frac{\beta_{h}}{\beta_{c}}
$$

which is exactly the Carnot efficiency.

Later, in Section F.2 we will need Lemma 2 to hold in a more general scenario, i.e. instead of the final battery state being $\rho_{\mathrm{W}}^{1}=(1-\varepsilon)\left|E_{k}\right\rangle\left\langle\left. E_{k}\right|_{\mathrm{W}}+\varepsilon \mid E_{j}\right\rangle\left\langle\left. E_{j}\right|_{\mathrm{W}}\right.$, we want to allow the final battery state to be any energy block-diagonal state with trace distance $\varepsilon$. Next we state and prove this generalized lemma.

Lemma 6. Consider all heat engines which extract near perfect work (see Definition 5), but allowing for any final battery state with a trace distance $\varepsilon$ to the ideal final pure state $\left|E_{k}\right\rangle\left\langle\left. E_{k}\right|_{\mathrm{W}}\right.$. Then according to the macroscopic second law of thermodynamics, the maximum efficiency of a heat engine, $\eta_{\max }$ is the Carnot efficiency

$$
\eta_{\max }=\sup _{\rho_{\text {Cold }}^{1} \in \mathcal{S}} \eta^{\text {mac }}\left(\rho_{\text {Cold }}^{1}\right)=1-\frac{\beta_{h}}{\beta_{c}},
$$

and when the final state of the cold bath $\rho_{\text {Cold }}^{1}$ is thermal, the supremum is only achieved for quasi-static heat engines (see Def. (6) and Eq. (69)).

Proof. Firstly, let us note that since the initial state $\rho_{\text {Coldw }}^{0}$ which we start out with is energy blockdiagonal, the final state has to also be block-diagonal. Therefore, given the product structure between the cold bath and battery, it is sufficient to consider the case when the final battery state is energy block-diagonal. Next, let us note that any final state $\rho_{\mathrm{W}}^{2}$ which is energy block-diagonal, and has trace distance $\varepsilon$ with $\left|E_{k}\right\rangle\left\langle\left. E_{k}\right|_{\mathrm{W}}\right.$ can be written as,

$$
\rho_{\mathrm{W}}^{2}=(1-\varepsilon)\left|E_{k}\right\rangle\left\langle\left. E_{k}\right|_{\mathrm{W}}+\varepsilon \rho_{\mathrm{W}}^{\mathrm{junk}}, \text { where } \rho_{\mathrm{W}}^{\mathrm{junk}}=\sum_{i} p_{i} \mid E_{i}\right\rangle\left\langle\left. E_{i}\right|_{\mathrm{W}}, \quad \sum_{i} p_{i}=1 \text { and } p_{k}=0 .\right.
$$


Next, one can calculate $W_{\text {ext }}$ given by the standard free energy condition, i.e.

$$
F\left(\tau_{\beta_{c}}\right)+F\left(\rho_{\mathrm{W}}^{0}\right) \geq F\left(\rho_{\text {Cold }}^{1}\right)+F\left(\rho_{\mathrm{W}}^{2}\right) .
$$

Using the identity $F(\rho)=\operatorname{tr}(\hat{H} \rho)-\beta^{-1} S(\rho)$, we have that

$$
F\left(\tau_{\beta_{c}}\right)+E_{j} \geq F\left(\rho_{\text {Cold }}^{1}\right)+(1-\varepsilon) E_{k}+\varepsilon \operatorname{tr}\left(\hat{H}_{\mathrm{W}} \rho_{\mathrm{W}}^{\mathrm{junk}}\right)-\beta_{h}^{-1} S\left(\rho_{\mathrm{W}}^{2}\right) .
$$

Substituting $W_{\text {ext }}=E_{k}-E_{j}$, and rearranging terms, we have

$$
(1-\varepsilon) W_{\text {ext }} \leq F\left(\tau_{\beta_{c}}\right)-F\left(\rho_{\text {Cold }}^{1}\right)+\beta_{h}^{-1} \Delta S-\varepsilon\left[\operatorname{tr}\left(\hat{H}_{\mathrm{W}} \rho_{\mathrm{W}}^{\mathrm{junk}}\right)-E_{j}\right] .
$$

Finally, by using the identity (in Eq. (54)) that $F(\rho)=\beta_{h}^{-1}\left[D\left(\rho \| \tau_{\beta_{h}}\right)-\ln Z_{\beta_{h}}\right]$, the maximum amount of extractable work is given by

$$
W_{\text {ext }}=(1-\varepsilon)^{-1} \beta_{h}^{-1} \cdot\left[D\left(\tau_{\beta_{c}} \| \tau_{\beta_{h}}\right)-D\left(\rho_{\text {Cold }}^{1} \| \tau_{\beta_{h}}\right)+\Delta S-\varepsilon \tilde{E}\right],
$$

where $\tilde{E}=\operatorname{tr}\left(\hat{H}_{\mathrm{W}} \rho_{\mathrm{W}}^{\mathrm{junk}}\right)-E_{j}$.

Following the steps in Lemma 5, in particular the derivations in Eq. (130) and (131), we have

$$
\eta_{\max }^{-1}=(1-\varepsilon) \cdot\left[1+\beta_{h} \inf _{\substack{\beta_{f} \\ \Delta C>0}} \frac{\Delta C}{D\left(\tau_{\beta_{c}} \| \tau_{\beta_{h}}\right)-D\left(\tau_{\beta_{f}} \| \tau_{\beta_{h}}\right)+\Delta S-\varepsilon \tilde{E}}\right] .
$$

To show Eq. (151) gives the Carnot efficiency, we show that 1) for non quasi-static cases where $\beta_{f}<\beta_{c}$, Carnot efficiency is not attained, and 2) in the quasi-static limit, Carnot efficiency is attained.

Let us first consider the case of extracting a non-vanishing amount of near perfect work, i.e. for all cases where $\beta_{f}<\beta_{c}$. Then near perfect work, by Def. 5 , corresponds to the limit $\varepsilon \rightarrow 0$,

$$
\begin{aligned}
\eta^{-1} & =\lim _{\varepsilon \rightarrow 0}(1-\varepsilon) \cdot\left[1+\beta_{h} \frac{\Delta C}{D\left(\tau_{\beta_{c}} \| \tau_{\beta_{h}}\right)-D\left(\tau_{\beta_{f}} \| \tau_{\beta_{h}}\right)+\Delta S-\varepsilon \tilde{E}}\right] \\
& =1+\beta_{h} \frac{\Delta C}{D\left(\tau_{\beta_{c}} \| \tau_{\beta_{h}}\right)-D\left(\tau_{\beta_{f}} \| \tau_{\beta_{h}}\right)} .
\end{aligned}
$$

In this limit, all terms involving $\varepsilon$ vanish, and the inverse efficiency has the same expression as the efficiency for perfect work. We already know from Lemma 4 that the infimum over $\beta_{f}$ cannot be obtained in this regime, since the inverse efficiency is strictly decreasing with $\beta_{f}$.

Therefore, again we are left with analyzing the quasi-static limit for this problem. Following the derivation in Eq. (139) for the quasi-static limit, we obtain

$$
\eta_{\max }^{-1}=1+\beta_{h} \lim _{g \rightarrow 0^{+}} \frac{\Delta C}{\left[D\left(\tau_{\beta_{c}} \| \tau_{\beta_{h}}\right)-D\left(\tau_{\beta_{f}} \| \tau_{\beta_{h}}\right)\right]} \cdot\left(1+\frac{\Delta S-\varepsilon \tilde{E}}{D\left(\tau_{\beta_{c}} \| \tau_{\beta_{h}}\right)-D\left(\tau_{\beta_{f}} \| \tau_{\beta_{h}}\right)}\right)^{-1},
$$

where $\varepsilon=\varepsilon(g)$ and note that requiring near perfect work implies that

$$
\lim _{g \rightarrow 0^{+}} \frac{\Delta S}{D\left(\tau_{\beta_{c}} \| \tau_{\beta_{h}}\right)-D\left(\tau_{\beta_{f}} \| \tau_{\beta_{h}}\right)}=0 .
$$

Next, we observe the relationship between $\varepsilon$ and $\Delta S$, in the regime where $\varepsilon$ is small. Given any $\varepsilon>0$ denoting the trace distance $d\left(\rho_{\mathrm{W}}^{2},\left|E_{k}\right\rangle\left\langle\left. E_{k}\right|_{\mathrm{W}}\right)=\varepsilon\right.$, the smallest amount of entropy that can be produced corresponds to $\Delta S=h_{2}(\varepsilon)$. This is because if we try to distribute the weight $\varepsilon$ over more energy eigenvalues, then by majorization the entropy only increases. But we also know that $\varepsilon \leq h_{2}(\varepsilon)$ for small values of $\varepsilon$, in particular over the regime $\varepsilon \in\left[0, \frac{1}{2}\right]$. Therefore, we have that in this regime, $\varepsilon \leq h_{2}(\varepsilon) \leq \Delta S$ holds. Therefore, we also know that

$$
\lim _{g \rightarrow 0^{+}} \frac{\varepsilon \tilde{E}}{D\left(\tau_{\beta_{c}} \| \tau_{\beta_{h}}\right)-D\left(\tau_{\beta_{f}} \| \tau_{\beta_{h}}\right)}=0,
$$

where $\varepsilon=\varepsilon(g)$. Plugging Eqns. (155) and (156) into Eq. (154), we have that the quasi-static efficiency is $\eta=1-\frac{\beta_{h}}{\beta_{c}}$. 


\section{E Efficiency of a nanoscopic quantum heat engine}

In this section, we will be applying the conditions for state transitions for nanoscale systems, as detailed in Section B.2. The reader will see that due to these extra constraints from the generalized free energies, the fundamental limitations on efficiency will differ greatly from those observed in Section D.

Firstly, in Section E.1, we show that the extraction of a positive amount of perfect work is impossible using the setup. In Section E.2, we show that this can be resolved by considering near perfect work instead. Then we find that:

(1) The maximum achievable efficiency is still the Carnot efficiency. This is proven in Section E.2.2.

(2) However, in the case of quasi-static heat engines, the Carnot efficiency cannot be achieved for all cold bath Hamiltonians. This is our main result, which is stated in Theorem 2, found in Section E.2.6. The results in Section E.2.4 and E.2.5 are more technical proofs, that pave the way for deriving this main result.

\section{E.1 Impossibility of extracting perfect work}

We will first show that with the general setup as described in Section A, no perfect work can ever be extracted. By this we mean that whenever $\varepsilon$ as defined in Eq.(42) equals zero, then for any value of $W_{\text {ext }}>0$, and for any final state $\rho_{\text {Cold }}^{1}$, the transition $\left|E_{j}\right\rangle\left\langle\left. E_{j}\right|_{\mathrm{W}} \otimes \tau_{\text {Cold }}^{0} \rightarrow \mid E_{k}\right\rangle\left\langle\left. E_{k}\right|_{\mathrm{W}} \otimes \rho_{\text {Cold }}^{1}\right.$ is not possible. Intuitively speaking, this occurs because the cold bath is initially in a state of full rank. Since thermal operations cannot decrease the rank of the system, therefore the final state of the cold bath $\rho_{\text {Cold }}^{1}$ must also be of full rank. By directly solving Eq. (55), for $W_{\text {ext }}$, we can find the an equation governing the amount of extractable work. To solve Eq. (55) for $W_{\text {ext }}$, we start by first using Eq. (56) to find an expression solely in term of the Rényi divergences and the fact that the bipartite thermal states are product states,

$$
D_{\alpha}\left(\tau_{\text {Cold }}^{0} \otimes \rho_{\mathrm{W}}^{0} \| \tau_{\text {ColdW }}^{h}\right) \geq D_{\alpha}\left(\rho_{\text {Cold }}^{1} \otimes \rho_{\mathrm{W}}^{1} \| \tau_{\text {ColdW }}^{h}\right) \quad \forall \alpha \geq 0 .
$$

Now using the additivity of the Rényi divergences, from (157) it follows

$$
D_{\alpha}\left(\rho_{\mathrm{W}}^{1} \| \tau_{\mathrm{W}}^{h}\right)-D_{\alpha}\left(\rho_{\mathrm{W}}^{0} \| \tau_{\mathrm{W}}^{h}\right) \leq D_{\alpha}\left(\tau_{\text {Cold }}^{0} \| \tau_{\text {Cold }}^{h}\right)-D_{\alpha}\left(\rho_{\text {Cold }}^{1} \| \tau_{\text {Cold }}^{h}\right) \quad \forall \alpha \geq 0 .
$$

By directly solving the L.H.S., we find that $W_{\text {ext }}$ must satisfy

$$
W_{\text {ext }} \leq k T_{\text {Hot }}\left[D_{\alpha}\left(\tau_{\text {Cold }}^{0} \| \tau_{\text {Cold }}^{h}\right)-D_{\alpha}\left(\rho_{\text {Cold }}^{1} \| \tau_{\text {Cold }}^{h}\right)\right] \quad \forall \alpha \geq 0 .
$$

Hence

$$
W_{\text {ext }} \leq k T_{\text {Hot }} \inf _{\alpha \geq 0}\left[D_{\alpha}\left(\tau_{\text {Cold }}^{0} \| \tau_{\text {Cold }}^{h}\right)-D_{\alpha}\left(\rho_{\text {Cold }}^{1} \| \tau_{\text {Cold }}^{h}\right)\right]
$$

where $\tau_{\text {Cold }}^{h}$ is the thermal state of the cold bath (according to the cold bath Hamiltonian $\hat{H}_{\text {Cold }}$ ), at temperature $T_{\text {Hot }}$ (since the surrounding hot bath is of temperature $T_{\text {Hot }}$ ). However from Eq. (56), $D_{0}\left(\tau_{\text {Cold }}^{0} \| \tau_{\text {Cold }}^{h}\right)=D_{0}\left(\rho_{\text {Cold }}^{1} \| \tau_{\text {Cold }}^{h}\right)$. Therefore according to Eq. (160), the amount of work extractable satisfies $W_{\text {ext }} \leq 0$.

We phrase this with more rigor in the following Lemmas 7 and 8, which proves that for perfect work, $W_{\text {ext }}>0$ is impossible. The proof holds for general initial states $\rho_{\text {Cold }}^{0}$ of full rank, in particular, they need not even be diagonal in the energy eigenbasis.

Lemma 7. For any $W_{\mathrm{ext}}>0$, consider the Hamiltonian $\hat{H}_{\mathrm{W}}$ given by Eq. (40). Then for any inverse temperature $\beta_{h}>0$, the thermal state $\tau_{\mathrm{W}}^{h}=\frac{1}{\operatorname{tr}\left[e^{-\beta_{h} \hat{H}_{\mathrm{W}}}\right]} e^{-\beta_{h} \hat{H}_{\mathrm{W}}}$ satisfies

$$
\operatorname{tr}\left[\left(\left|E_{j}\right\rangle\left\langle\left. E_{j}\right|_{\mathrm{W}}-\mid E_{k}\right\rangle\left\langle\left. E_{k}\right|_{\mathrm{W}}\right) \tau_{\mathrm{W}}^{h}\right]>0\right.
$$


Proof. Follows directly from the definitions. Since $W_{\text {ext }}>0$, we know that $E_{j}^{\mathrm{W}}<E_{k}^{\mathrm{W}}$. Evaluating the quantity above gives $\frac{1}{\operatorname{tr}\left[e^{-\beta_{h} \tilde{H}_{\mathrm{W}}}\right]} \cdot\left(e^{-\beta_{h} E_{j}^{\mathrm{W}}}-e^{-\beta_{h} E_{k}^{\mathrm{W}}}\right)>0$.

Lemma 8. Consider any general quantum state $\rho_{\text {Cold }}^{0}$ of full rank. Then for any $\rho_{\text {Cold }}^{1}$, the transition from $\rho_{\text {Cold }}^{0} \otimes \rho_{\mathrm{W}}^{0} \rightarrow \rho_{\text {Cold }}^{1} \otimes \rho_{\mathrm{W}}^{1}$ is not possible via catalytic thermal operations if

$$
\operatorname{tr}\left[\left(\Pi_{\rho_{\mathrm{W}}^{0}}-\Pi_{\rho_{\mathrm{W}}^{1}}\right) \tau_{\mathrm{W}}^{h}\right]>0,
$$

where $\Pi_{\rho}$ is the projector onto the support of state $\rho$, and $\tau_{\mathrm{W}}^{h}$ is the thermal state of the battery at the initial hot bath temperature.

Proof. One can show this by invoking the quantum second law for $\alpha=0$ [7], which says that if $\rho_{\text {in }} \rightarrow \rho_{\text {out }}$ is possible via catalytic thermal operations, then

$$
D_{0}\left(\rho_{\text {in }} \| \tau\right) \geq D_{0}\left(\rho_{\text {out }} \| \tau\right),
$$

where $\tau$ is the thermal state of the system at bath temperature, and

$$
D_{0}(\rho \| \sigma)=\lim _{\alpha \rightarrow 0^{+}} \frac{1}{\alpha-1} \ln \operatorname{tr}\left[\rho^{\alpha} \sigma^{1-\alpha}\right]=-\ln \operatorname{tr}\left[\Pi_{\rho} \sigma\right],
$$

is defined for arbitrary quantum states $\rho, \sigma$. Applying this law with $\rho_{\text {in }}=\rho_{\mathrm{W}}^{0} \otimes \rho_{\text {Cold }}^{0}$ and $\rho_{\text {out }}=$ $\rho_{\mathrm{W}}^{1} \otimes \rho_{\text {Cold }}^{1}$, we arrive at

$$
D_{0}\left(\rho_{\mathrm{W}}^{0} \| \tau_{\mathrm{W}}^{h}\right)-D_{0}\left(\rho_{\mathrm{W}}^{1} \| \tau_{\mathrm{W}}^{h}\right) \geq D_{0}\left(\rho_{\text {Cold }}^{1} \| \tau_{\text {Cold }}^{h}\right)-D_{0}\left(\rho_{\text {Cold }}^{0} \| \tau_{\text {Cold }}^{h}\right),
$$

where $\tau_{\text {Cold }}^{h}$ and $\tau_{\mathrm{W}}^{h}$ are thermal states of the cold bath and battery at the temperature of surrounding hot bath $\left(T_{\text {Hot }}\right)$ respectively. Since $\rho_{\text {Cold }}^{0}$ have full rank, and since $\tau_{\text {Cold }}^{h}$ is normalized, therefore according to Eq. (164), $D_{0}\left(\rho_{\text {Cold }}^{0} \| \tau_{\text {Cold }}^{h}\right)=0$. Furthermore, since the $\alpha$-Rényi divergence $D_{0}$ is non-negative, therefore the r.h.s. of Eq. (165) is lower bounded by 0 . Thus, we have

$$
\operatorname{tr}\left[\left(\Pi_{\rho_{\mathrm{W}}^{0}}-\Pi_{\rho_{\mathrm{W}}^{1}}\right) \tau_{\mathrm{W}}^{h}\right] \leq 0 .
$$

Since this is a necessary condition for state transformations, we arrive at the conclusion that: when Eq. (166) is violated, state transformations are not possible. But from Lemma 7, any type of perfect work extraction violates Eq. (166). Therefore, in this setting, perfect work extraction is always impossible.

To summarize, Lemma 8 implies that if the initial state of the cold bath is thermal, and therefore of full rank, then any work extraction scheme via thermal operations bringing $\rho_{\mathrm{W}}^{0}=|j\rangle\left\langle\left. j\right|_{\mathrm{W}}\right.$ to $\rho_{\mathrm{W}}^{1}=$ $|k\rangle\left\langle\left. k\right|_{\mathrm{W}}\right.$ where $W_{\text {ext }}=E_{k}^{\mathrm{W}}-E_{j}^{\mathrm{W}}>0$ is not possible. In general, we see that if $\Pi_{\rho_{\mathrm{W}}^{0}} \neq \Pi_{\rho_{\mathrm{W}}^{1}}$, then when transition $\rho_{\mathrm{W}}^{0}$ to $\rho_{\mathrm{W}}^{1}$ is possible, transition $\rho_{\mathrm{W}}^{1}$ to $\rho_{\mathrm{W}}^{0}$ is not. Consequentially, we will have to consider near perfect work at the nano regime.

\section{E.2 Efficiency for extracting near perfect work}

As we have just seen in the previous Section E.1, we cannot extract perfect work. Due to the impossibility result, we consider the relaxation of extracting near perfect work in the nanoscale setting.

- We begin by evaluating the expression for efficiency according to the nanoscopic laws of thermodynamics, given a final state of the cold bath, and comparing it to the expression according to macroscopic laws of thermodynamics. This is done in Sections E.2.1 and E.2.2, and the relation between two efficiencies are summarized in Eq. (177). Since the nanoscopic efficiency is always smaller than the macroscopic efficiency, which attains Carnot efficiency only in the limit $\Delta C \rightarrow 0$, it will be a necessary condition to consider this limit if we want to achieve the Carnot efficiency, when considering nanoscopic laws of thermodynamics. 
- We analyze the quasi-static regime, focusing on the special case where the cold bath consists of $n$ qubits. Since the quasi-static limit corresponds to the case of small $g>0$, and $\varepsilon$ also has to be arbitrarily small for near perfect work extraction, we perform Taylor expansion of the analytical expressions for $W_{\text {ext }}$ and $\Delta C$ w.r.t. $g$ and $\varepsilon$. This is done in Section E.2.3.

- In Section E.2.4, we identify how to choose $\varepsilon(g)$ such that it corresponds to drawing near perfect work in the quasi-static limit. We first begin by observing that any continuous function $\varepsilon(g)$ that vanishes in the limit $g \rightarrow 0$ can be characterized with a real-valued parameter $\bar{\kappa}$ that determines how quickly $\varepsilon$ goes to zero. This is shown in Lemma 11. In Lemma 12, we show that near perfect work is drawn only if $\bar{\kappa} \in[0,1]$.

- Lemma 12 gives us the analytical expression and minimization range in order to evaluate $W_{\text {ext }}$, according to Eq. (230). In Section E.2.5, we show how one can evaluate this optimization problem, by comparing the stationary points and endpoints of the function $\frac{\alpha B_{\alpha}}{\alpha-1}$ that gives the leading term in Eq. (230). Lemma 13 proves a technical property of the first derivative of this function. Using it, we prove in Lemma 14 that one can always choose $\varepsilon(g)$ with some $\bar{\kappa}<1$ such that the infimum of $\frac{\alpha B_{\alpha}}{\alpha-1}$ is obtained at either $\alpha=\bar{\kappa}$ or $\alpha \rightarrow \infty$.

- Finally, in Section E.2.6, we use the results in Section E.2.5 regarding the evaluation of $W_{\text {ext }}$ to find the efficiency in the quasi-static limit.

\section{E.2.1 An explicit expression for $W_{\text {ext }}$}

Our first task is to work out an explicit expression for $W_{\text {ext }}$ depending on the initial and final states of the cold bath, $\varepsilon$ and hot bath (inverse) temperature $\beta_{h}$. Such as expression is found by applying the generalized second laws as detailed in Section B.2.

\section{Lemma 9. Consider the transition}

$$
\tau_{\text {Cold }}^{0} \otimes \rho_{\mathrm{W}}^{0} \rightarrow \rho_{\text {Cold }}^{1} \otimes \rho_{\mathrm{W}}^{1} \quad \text { with } \quad \varepsilon>0 .
$$

where $\rho_{\mathrm{W}}^{0}$ and $\rho_{\mathrm{W}}^{1}$ are defined in Eqs. (41), (42) respectively. Let $W_{\text {ext }}$ denote the maximum possible value such that Eq. (167) is possible via catalytic thermal operations, with a thermal bath of inverse temperature $\beta_{h}$. Let $\beta_{c}>\beta_{h}$. Then the final state $\rho_{\text {Cold }}^{1}=\sum_{i} p_{i}^{\prime}\left|E_{i}\right\rangle\left\langle\left. E_{i}\right|_{\text {Cold }}\right.$ is block-diagonal in the energy eigenbasis, and

$$
\begin{aligned}
W_{\mathrm{ext}} & =\inf _{\alpha \geq 0} W_{\alpha}, \\
W_{\alpha} & =\frac{1}{\beta_{h}(\alpha-1)}\left[\ln \left(A-\varepsilon^{\alpha}\right)-\alpha \ln (1-\varepsilon)\right], \\
A & =\frac{\sum_{i} p_{i}^{\alpha} q_{i}^{1-\alpha}}{\sum_{i} p_{i}^{\prime \alpha} q_{i}^{1-\alpha}},
\end{aligned}
$$

where $p_{i}=\frac{e^{-\beta_{c} E_{i}}}{Z_{\beta_{C}}}, q_{i}=\frac{e^{-\beta_{h} E_{i}}}{Z_{\beta_{h}}}$, and $p_{i}^{\prime}$ are the probability amplitudes of state $\rho_{\text {Cold }}^{1}$ when written in the energy eigenbasis of $\hat{H}_{\text {Cold }}$. The quantities $W_{1}$ and $W_{\infty}$ are defined by taking the limit $\alpha \rightarrow 1,+\infty$ respectively.

Proof. Eq. (55) is necessary and sufficient for Eq. (167) to be satisfied. We can apply the additivity property of the Rényi divergence, to Eq. (55) to find

$$
D_{\alpha}\left(\rho_{\mathrm{W}}^{0} \| \tau_{\mathrm{W}}^{h}\right)+D_{\alpha}\left(\tau_{\beta_{c}} \| \tau_{\beta_{h}}\right) \geq D_{\alpha}\left(\rho_{\mathrm{W}}^{1} \| \tau_{\mathrm{W}}^{h}\right)+D_{\alpha}\left(\rho_{\text {Cold }}^{1} \| \tau_{\beta_{h}}\right),
$$

where $\tau_{\beta_{h}}$ and $\tau_{\mathrm{W}}^{h}$ are the thermal states with Hamiltonians $\hat{H}_{\text {Cold }}$ and $\hat{H}_{\mathrm{W}}$ respectively, at inverse temperature $\beta_{h}$. From Eq. (171) it follows,

$$
\begin{aligned}
\frac{-1}{\alpha-1} \ln \left[\varepsilon^{\alpha}+(1-\varepsilon)^{\alpha} e^{-\beta\left(E_{k}-E_{j}\right)(1-\alpha)}\right] & \geq D_{\alpha}\left(\rho_{\text {Cold }}^{1} \| \tau_{\beta_{h}}\right)-D_{\alpha}\left(\tau_{\beta_{c}} \| \tau_{\beta_{h}}\right) \\
& =\frac{-1}{\alpha-1} \ln A .
\end{aligned}
$$


By examining the three cases $\alpha<1, \alpha>1$ separately, have find

$$
\begin{array}{ll}
\varepsilon^{\alpha}+(1-\varepsilon)^{\alpha} e^{-\beta\left(E_{k}-E_{j}\right)(1-\alpha)} \geq A & \text { if } \alpha<1 \\
\varepsilon^{\alpha}+(1-\varepsilon)^{\alpha} e^{-\beta\left(E_{k}-E_{j}\right)(1-\alpha)} \leq A & \text { if } \alpha>1
\end{array}
$$

where note that $A$ is independent of $E_{k}$ and $E_{j}$. Now note that the largest value of $E_{k}-E_{j}$ in Eq. (174), is obtained when the inequalities hold with equality. Thus, since these equations have to hold for all $\alpha \in[0, \infty]$, the largest amount of work we can extract corresponds to the value of $E_{k}-E_{j}$ for which Eq. (174) holds for all $\alpha \in[0, \infty]$, with an equality for at least one particular value of $\alpha \in[0, \infty]$. In other words $W_{\text {ext }}$ is given by Eq. (168), and $W_{\alpha}$ satisfies,

$$
\varepsilon^{\alpha}+(1-\varepsilon)^{\alpha} e^{-\beta W_{\alpha}(1-\alpha)}=A .
$$

Note that due to the continuity of the Rényi divergences in the neighbourhood of one, this case follows by continuity. Solving Eq. (175) for $W_{\alpha}$ gives us Eq. (169).

As we will see later there exist $\rho_{\text {Cold }}^{1}$ such that, $W_{\text {ext }}$ given by Eq. (168), has a solution (i.e. $W_{\text {ext }}>0$ ) for any $\varepsilon>0$. We can use this to write down an explicit solution to the maximization problem Eq. (62). Using Eqs. (62), (77) and Lemma 9, we conclude

$$
\eta^{\text {nano }}\left(\rho_{\text {Cold }}^{1}\right)=\left(1-\varepsilon+\frac{\Delta C\left(\rho_{\text {Cold }}^{1}\right)}{\inf _{\alpha \geq 0} W_{\alpha}\left(\rho_{\text {Cold }}^{1}\right)}\right)^{-1}
$$

where $W_{\alpha}$ is given by Eqs. (169), (170) and recall $\Delta C$ can be found in Eq. (74). From Eqs. (176), (169), (170), we see that the optimization problem $\sup _{\rho_{\text {Cold }}^{1}} \eta^{\text {nano }}\left(\rho_{\text {Cold }}^{1}\right)$ is still a formidable task. In the next section, see will show that we can use the results from Section D, to drastically simplify the problem.

\section{E.2.2 An upper bound for the efficiency}

Before moving on to solving the nanoscale efficiency explicitly, we will first use the results of Section D.3 to find upper bounds for the efficiency in the nanoscale regime, in the context of extracting near perfect work (Def. 5).

Recall how we have discussed in comparing Sections B.1 and B.2, that the solution for the family of free entropies $F_{\alpha}$, in the case of $F_{1}$ is simply the non-equilibrium free energy. Therefore, from Lemma 9, it follows that $W_{1}$ is simply the maximum amount of extractable work according to Eq. (49). From Eqs. (65), (77),

$$
\eta^{\mathrm{mac}}\left(\rho_{\text {Cold }}^{1}\right)=\left(1-\varepsilon+\frac{\Delta C\left(\rho_{\text {Cold }}^{1}\right)}{W_{1}\left(\rho_{\text {Cold }}^{1}\right)}\right)^{-1} .
$$

One can now compare Eq. (177) with Eq. (176), and note that for any $\rho_{\text {Cold }}^{1} \in \mathcal{S}$, we have $W_{1}\left(\rho_{\text {Cold }}^{1}\right) \geq$ $\inf _{\alpha \geq 0} W_{\alpha}\left(\rho_{\text {Cold }}^{1}\right)$. Therefore, we conclude that for any $\rho_{\text {Cold }}^{1} \in \mathcal{S}$,

$$
\eta^{\text {nano }}\left(\rho_{\text {Cold }}^{1}\right) \leq \eta^{\text {mac }}\left(\rho_{\text {Cold }}^{1}\right) .
$$

Eq. (178) in conjunction with Lemma 5 has an important consequence. Namely,

$$
\sup _{\rho_{\text {Cold }}^{1} \in \mathcal{S}} \eta^{\text {nano }}\left(\rho_{\text {Cold }}^{1}\right) \leq 1-\beta_{h} / \beta_{c}
$$

with equality only if the state $\rho_{\text {Cold }}^{1}$ that solves the supremum is the limiting case where it tends to the initial state of the cold bath, $\rho_{\text {Cold }}^{1} \rightarrow \rho_{\text {Cold }}^{0}$. Therefore, in order to see whether we can still achieve the Carnot efficiency, we will consider the quasi-static regime in the rest of Section E. 


\section{E.2.3 Evaluating near perfect work in the quasi-static heat engine}

In light of the results from the previous section, we will now calculate the near perfect work $W_{\text {ext }}$ for quasi-static heat engines, i.e. the case where $\varepsilon, g \ll 1$. Specifically, we make the following assumption about the cold bath Hamiltonian:

(A.5) The Hamiltonian is taken to be of $n$ qubits:

$$
\hat{H}_{\mathrm{Cold}}=\sum_{k=1}^{n} \mathbb{1}^{\otimes(k-1)} \otimes \hat{H}_{\mathrm{c}, k} \otimes \mathbb{1}^{\otimes(n-k)}, \quad \text { where } \quad \hat{H}_{\mathrm{c}, k}=\bar{E}_{k}\left|\bar{E}_{k}\right\rangle\left\langle\bar{E}_{k}\right|,
$$

and $\bar{E}_{k}>0$ is the energy gap of the $k$-th qubit. Here for simplify, we have chosen w.l.o.g. the ground state of each qubit to have an eigenvalue equal to zero.

The tensor product structure in Assumption (A.5) allows us to simplify $\rho_{\text {Cold }}^{0}$, to

$$
\rho_{\text {Cold }}^{0}=\bigotimes_{i=1}^{n} \tau_{i, \beta_{c}},
$$

where $\tau_{i, \beta_{c}}$ is the thermal state of $i$ th qubit Hamiltonian $\hat{H}_{i, \mathrm{c}}$ at inverse temperature $\beta_{c}$. For the simplicity of following proofs, we present them in the special case of identical qubits, i.e. that $\bar{E}_{i}=E$ for all $1 \leq i \leq n$. This means Eq. (181) can be reduced to

$$
\rho_{\text {Cold }}^{0}=\tau_{\beta_{c}}^{\otimes n} .
$$

Furthermore, since we consider quasi-static heat engines, the output state is

$$
\rho_{\text {Cold }}^{1}=\tau_{\beta_{f}}^{\otimes n},
$$

with $\beta_{f}=\beta_{c}-g$,where $0<g \ll 1$. Eq. (180) together with Eq. (183) allows us to further simplify Eq. (170) to

$$
A=\left(\frac{\sum_{i} p_{i}^{\alpha} q_{i}^{1-\alpha}}{\sum_{i} p_{i}^{\prime \alpha} q_{i}^{1-\alpha}}\right)^{n},
$$

where $p_{i}=\frac{e^{-\beta_{c} E \delta_{i, 0}}}{Z_{\beta_{c}}}, p_{i}^{\prime}=\frac{e^{-\beta_{f} E \delta_{i, 0}}}{Z_{\beta_{f}}}, q_{i}=\frac{e^{-\beta_{h} E \delta_{i, 0}}}{Z_{\beta_{h}}}$, with $\delta_{1,0}=0, \delta_{1,1}=1$, are the probabilities of thermal states (different temperatures) for the qubit Hamiltonian $\hat{H}_{\mathrm{c}}$. The proof follows along the same lines as the proof to Lemma 9, but now noting that in Eq. (171) we can replace $D_{\alpha}\left(\tau_{\text {Cold }} \| \tau_{\beta_{h}}\right)$ and $D_{\alpha}\left(\rho_{\text {Cold }}^{1} \| \tau_{\beta_{h}}\right)$ with $n D_{\alpha}\left(\tau_{\beta_{c}} \| \tau_{\beta_{h}}\right)$ and $n D_{\alpha}\left(\tau_{\beta_{f}} \| \tau_{\beta_{h}}\right)$ respectively. This follows from the additivity property of the Rényi divergences. After proving the special case of identical qubits, we show in Theorem 2 that it can be extended to non-identical qubits as generally described by Assumption (A.5).

Since we are dealing with near perfect work and quasi-static heat engines, both $g>0$ and $\varepsilon>0$ are infinitesimally small. Thus with the goal in find of finding a solution for $W_{\text {ext }}$ from Eqs. (168), (169), and (184); we will proceed to find an expansion of $W_{\alpha}$ for small $\varepsilon$ and $g$.

\section{i) The expansion of $A$ in a quasi-static heat engine}

To simplify our calculations of $W_{\text {ext }}$, especially that of efficiency, it is important to express $A$ in Eq. (184) in terms of its first order expansion w.r.t. the parameter $g$. Recall that this parameter $g=\beta_{c}-\beta_{f}$ is the difference of inverse temperature between the initial and final state of the cold bath.

Firstly, note that for any integer $n$, the expression in Eq. (184) evaluates to $\left.A\right|_{g=0}=1$. This is because at $g=0, \beta_{f}=\beta_{c}$ and therefore the probabilities $p_{i}, p_{i}^{\prime}$ are identical. To obtain an approximation in 
the regime $0<g \ll 1$, we derive

$$
\begin{aligned}
\frac{d A}{d g} & =-n\left(\sum_{i} p_{i}^{\alpha} q_{i}^{1-\alpha}\right)^{n}\left(\sum_{i} p_{i}^{\prime \alpha} q_{i}^{1-\alpha}\right)^{-n-1}\left[\sum_{i} \alpha p_{i}^{\prime \alpha-1} q_{i}^{1-\alpha} \frac{d p_{i}^{\prime}}{d g}\right] \\
& =-\alpha n A\left(\sum_{i} p_{i}^{\prime \alpha} q_{i}^{1-\alpha}\right)^{-1}\left[\sum_{i} p_{i}^{\prime \alpha} q_{i}^{1-\alpha}\left(\bar{E}_{i}-\left\langle\hat{H}_{\mathrm{c}}\right\rangle_{\beta_{f}}\right)\right] .
\end{aligned}
$$

The first inequality holds by noticing that only the probabilities $p_{i}^{\prime}$ depend on $g$, which means only the denominator in Eq. (184) is differentiated, using the chain rule

$$
\frac{d A\left(\left\{p_{i}^{\prime}\right\}\right)}{d g}=\sum_{i} \frac{d A\left(\left\{p_{i}^{\prime}\right\}\right)}{d p_{i}^{\prime}} \frac{d p_{i}^{\prime}}{d g} .
$$

The equality in Eq. (186) makes use of the fact that $\frac{d p_{i}^{\prime}}{d g}=-\frac{d p_{i}^{\prime}}{d \beta_{f}}=p_{i}^{\prime}\left(\bar{E}_{i}-\left\langle\hat{H}_{\mathrm{c}}\right\rangle_{\beta_{f}}\right)$ as derived in Eq. (102). Evaluated at $g=0$, implies that $p_{i}^{\prime}=p_{i}$, and therefore this gives

$$
\begin{aligned}
\left.\frac{d A}{d g}\right|_{g=0} & =\alpha n B_{\alpha}, \text { where } \\
B_{\alpha} & =\frac{1}{\sum_{i} p_{i}^{\alpha} q_{i}^{1-\alpha}} \sum_{i} p_{i}^{\alpha} q_{i}^{1-\alpha}\left(\left\langle\hat{H}_{\mathrm{c}}\right\rangle_{\beta_{c}}-\bar{E}_{i}\right) .
\end{aligned}
$$

Recall that $p_{i}, q_{i}$ are probabilities of the thermal states of $\hat{H}_{\mathrm{c}}$, at inverse temperatures $\beta_{c}, \beta_{h}$ respectively. With this, we can write the expansion of $A$ with respect to $g$ as

$$
A=1+\alpha n g B_{\alpha}+\Theta\left(g^{2}\right) .
$$

Later on, we will also need to evaluate the derivative of $B_{\alpha}$ w.r.t. $\alpha$. This quantity, when evaluated at $\alpha=1$, has a close relation to the change in average energy of the cold bath (per copy), $\frac{\Delta C}{n}$.

Lemma 10. Let

$$
\Delta C^{\prime}\left(\beta_{c}\right):=\left.\frac{d}{d g} \Delta C\left(\beta_{f}\right)\right|_{g=0}
$$

where recall $\beta_{f}=\beta_{c}-g$. Then

$$
B_{1}^{\prime}=\left.\frac{d B_{\alpha}}{d \alpha}\right|_{\alpha=1}=\frac{\beta_{c}-\beta_{h}}{n} \Delta C^{\prime}\left(\beta_{f}\right)=\left(\beta_{c}-\beta_{h}\right) \cdot \operatorname{var}\left(\hat{H}_{\mathrm{c}}\right)_{\beta_{c}} .
$$

Proof. From the definition of $\Delta C$ (Eq. (74)) and using Eqs. (180), (181), (183), we have

$$
\frac{\Delta C}{n}=\operatorname{tr}\left[\left(\tau_{\beta_{f}}-\tau_{\beta_{c}}\right) \hat{H}_{\mathrm{c}}\right] .
$$

Recalling that $\beta_{f}=\beta_{c}-g$ and using Eq. (112), from Eq. (193) it follows

$$
\frac{1}{n} \Delta C^{\prime}\left(\beta_{c}\right)=\left.\frac{1}{n} \frac{d \Delta C}{d g}\right|_{g=0}=-\left.\frac{1}{n} \frac{d \Delta C}{d \beta_{f}}\right|_{\beta_{f}=\beta_{c}}=\operatorname{var}\left(\hat{H}_{\mathrm{c}}\right)_{\beta_{c}} .
$$

Now, let us evaluate the partial derivative of $B_{\alpha}$ w.r.t. $\alpha$. Denoting $r_{i}=\frac{p_{i}}{q_{i}}$, and invoking the chain rule of derivatives for Eq. (189)

$$
\begin{aligned}
\frac{d B_{\alpha}}{d \alpha}= & \left(\sum_{i} p_{i}^{\alpha} q_{i}^{1-\alpha}\right)^{-2}\left\{\left[\sum_{i} q_{i} r_{i}^{\alpha} \ln r_{i}\left(\left\langle\hat{H}_{\mathrm{c}}\right\rangle_{\beta_{c}}-\bar{E}_{i}\right)\right]\left[\sum_{i} p_{i}^{\alpha} q_{i}^{1-\alpha}\right]\right. \\
& \left.-\left[\sum_{i} q_{i} r_{i}^{\alpha} \ln r_{i}\right]\left[\sum_{i} p_{i}^{\alpha} q_{i}^{1-\alpha}\left(\left\langle\hat{H}_{\mathrm{c}}\right\rangle_{\beta_{c}}-\bar{E}_{i}\right)\right]\right\} .
\end{aligned}
$$


Substituting $\alpha=1$ into Eq. (195), we obtain that $\sum_{i} p_{i}^{\alpha} q_{i}^{1-\alpha}=1$. Also, $\sum_{i} p_{i}^{\alpha} q_{i}^{1-\alpha}\left(\left\langle\hat{H}_{\mathrm{c}}\right\rangle_{\beta_{c}}-\bar{E}_{i}\right)=0$ while the factor multiplied in front is finite. Therefore, we are left with the terms

$$
\begin{aligned}
B_{1}^{\prime} & =\sum_{i} p_{i} \ln r_{i}\left(\left\langle\hat{H}_{\mathrm{c}}\right\rangle_{\beta_{c}}-\bar{E}_{i}\right) \\
& =\sum_{i} p_{i}\left[\ln \frac{Z_{h}}{Z_{c}}+\left(\beta_{h}-\beta_{c}\right) \bar{E}_{i}\right]\left(\left\langle\hat{H}_{\mathrm{c}}\right\rangle_{\beta_{c}}-\bar{E}_{i}\right) \\
& =\left(\beta_{c}-\beta_{h}\right) \operatorname{var}\left(\hat{H}_{\mathrm{c}}\right)_{\beta_{c}} \\
& =\frac{\beta_{c}-\beta_{h}}{n} \Delta C^{\prime}\left(\beta_{c}\right)
\end{aligned}
$$

The second equality comes from substituting $r_{i}=\frac{p_{i}}{q_{i}}=e^{\left(\beta_{h}-\beta_{c}\right) \bar{E}_{i}} \cdot Z_{h} / Z_{c}$. In the third equality, $\ln \frac{Z_{h}}{Z_{c}}$ is brought out of the summation, while the summation yields 0 . Subsequently, we invoke $\sum_{i} p_{i} \bar{E}_{i}\left(\left\langle\hat{H}_{\mathrm{c}}\right\rangle_{\beta_{c}}-\bar{E}_{i}\right)=\left\langle\hat{H}_{\mathrm{c}}\right\rangle_{\beta_{c}}^{2}-\left\langle\hat{H}_{\mathrm{c}}^{2}\right\rangle_{\beta_{c}}=-\operatorname{var}\left(\hat{H}_{\mathrm{c}}\right)_{\beta_{c}}$.

\section{ii) The expansion of $W_{\alpha}$ in the quasi-static heat engine}

In the following we proceed to derive an expansion of $W_{\alpha}$ valid for small $g$, and $\varepsilon$. Note that $W_{1}$ is defined through continuity to be the limit of the Rényi divergences at $\alpha \rightarrow 1$, and the small $\varepsilon$ and $g$ expansion does not hold for $\alpha=0$, we shall have to examine $W_{1}$ and $W_{0}$ separately.

In the following and throughout the manuscript, we will use the notation $x \in(y, \infty]$ to indicate that the expression whose input $x$ in being referred to holds for $x \in(y, \infty)$ and for the limit case $\lim _{x \rightarrow+\infty}$. Similarly, we use the notation $x \in[y, \infty]$ when referring to an expression which holds for $x \in[y, \infty)$ and for $\lim _{x \rightarrow+\infty}$.

(A) For $\varepsilon>0, \alpha \in(0,1) \cup(1, \infty]$.

We start with the case $\varepsilon>0, \alpha \in(0,1) \cup(1, \infty)$ :

$$
\begin{aligned}
W_{\alpha} & =\frac{1}{\beta_{h}(\alpha-1)}\left[\ln \left(A-\varepsilon^{\alpha}\right)-\alpha \ln (1-\varepsilon)\right] \\
& =\frac{1}{\beta_{h}(\alpha-1)}\left[\ln \left(1+\alpha n g B_{\alpha}+\Theta\left(g^{2}\right)-\varepsilon^{\alpha}\right)-\alpha \ln (1-\varepsilon)\right] \\
& =\frac{1}{\beta_{h}(\alpha-1)}\left[\alpha n g B_{\alpha}+\Theta\left(g^{2}\right)-\varepsilon^{\alpha}+\Theta\left(\varepsilon^{2 \alpha}\right)+\Theta\left(g \varepsilon^{\alpha}\right)-\alpha\left(-\varepsilon+\Theta\left(\varepsilon^{2}\right)\right)\right], \\
& =\frac{1}{\beta_{h}(\alpha-1)}\left[\alpha n g B_{\alpha}-\varepsilon^{\alpha}+\alpha \varepsilon\right]+\Theta\left(g^{2}\right)+\Theta\left(\varepsilon^{2 \alpha}\right)+\Theta\left(g \varepsilon^{\alpha}\right)+\Theta\left(\varepsilon^{2}\right) .
\end{aligned}
$$

In the second equality, we have used the expansion of $A$ derived in Eq. (190). In the third equality, we use the Mercator series

$$
\ln (1+x)=\sum_{k=1}^{\infty} \frac{(-1)^{k+1}}{k} x^{k}, \quad|x|<1,
$$

to expand both of the natural logarithms in line Eq. (201). The order terms of $\Theta\left(g^{3}\right), \Theta\left(g^{4}\right), \Theta\left(g^{2} \varepsilon^{\alpha}\right)$ vanish because they are of higher order compared with $\Theta\left(g^{2}\right)$ and $\Theta\left(g \varepsilon^{\alpha}\right)$. The last equality occurs because $c \Theta(g(x))=\Theta(g(x))$ for any $c \in \mathbb{R} \backslash 0$.

Finally, we consider the limit case $\alpha \rightarrow \infty$. By direct calculation using the expression in line Eq. (201), we find

$$
\lim _{\alpha \rightarrow+\infty} W_{\alpha}=\frac{1}{\beta_{h}}\left[n g \lim _{\alpha \rightarrow+\infty} B_{\alpha}+\varepsilon\right]+\Theta\left(g^{2}\right)+\Theta\left(\varepsilon^{2}\right),
$$


which is identical to the expression one obtains by taking the limit $\alpha \rightarrow+\infty$ in Eq. (204). We thus conclude that for $\varepsilon>0, \alpha \in(0,1) \cup(1, \infty]$,

$$
W_{\alpha}=\frac{1}{\beta_{h}(\alpha-1)}\left[\alpha n g B_{\alpha}-\varepsilon^{\alpha}+\alpha \varepsilon\right]+\Theta\left(g^{2}\right)+\Theta\left(\varepsilon^{2 \alpha}\right)+\Theta\left(g \varepsilon^{\alpha}\right)+\Theta\left(\varepsilon^{2}\right) .
$$

(B) For $\varepsilon>0, \alpha=1$

We are now interested in finding a small $\varepsilon>0, g>0$ expansion for $W_{1}$, which is defined through continuity of the Rényi divergences. Going back to Eq. (171), note that $W_{1}$ is the maximum value such that Eq. (171) holds with equality, when all $D_{\alpha}$ terms in Eq. (171) are evaluated at $\alpha \rightarrow 1$. Recall that $\lim _{\alpha \rightarrow 1} D_{\alpha}(\rho \| \tau)=D(\rho \| \tau)$ (see Eq. (59)), the relative entropy we have derived in Section D. Therefore, one can write an equation for $W_{1}$ in a more compact form: $W_{1}$ is the value such that

$$
n \cdot\left[\left\langle\hat{H}_{\mathrm{c}}\right\rangle_{\beta_{c}}-\frac{1}{\beta_{h}} S\left(\beta_{c}\right)\right]=n \cdot\left[\left\langle\hat{H}_{\mathrm{c}}\right\rangle_{\beta_{f}}-\frac{1}{\beta_{h}} S\left(\beta_{f}\right)\right]+(1-\varepsilon) W_{1}-\frac{1}{\beta_{h}} \mathrm{~h}_{2}(\varepsilon),
$$

where $\left\langle\hat{H}_{\mathrm{c}}\right\rangle_{\beta_{c}}$ is the mean energy evaluated at temperature $T_{\text {Cold }}, S\left(\beta_{c}\right)$ is the von Neumann entropy of the state $\tau_{\beta_{c}}$, and $h_{2}(\varepsilon)$ is the binary entropy function. Rearranging Eq. (208), we get

$$
W_{1}=\frac{1}{1-\varepsilon}\left[n\left\langle\hat{H}_{\mathrm{c}}\right\rangle_{\beta_{c}}-n\left\langle\hat{H}_{\mathrm{c}}\right\rangle_{\beta_{f}}-n \frac{1}{\beta_{h}}\left(S\left(\beta_{c}\right)-S\left(\beta_{f}\right)\right)+\frac{1}{\beta_{h}} \mathrm{~h}_{2}(\varepsilon)\right] .
$$

We can expand (209) using a power law expansion in $g$ and $\varepsilon$ for the terms in Eq.(209), obtaining

$$
W_{1}=\left[1+\varepsilon+\Theta\left(\varepsilon^{2}\right)\right] \cdot\left[\left.n \frac{d\left(-\left\langle\hat{H}_{\mathrm{c}}\right\rangle_{\beta_{f}}+\beta_{h}^{-1} S\left(\beta_{f}\right)\right)}{d g}\right|_{g=0} g+\Theta\left(g^{2}\right)+\frac{1}{\beta_{h}} \mathrm{~h}_{2}(\varepsilon)\right] .
$$

To proceed, we recall that $\beta_{f}=\beta_{c}-g$ and evaluate the term

$$
\begin{aligned}
\left.\frac{d\left(-\left\langle\hat{H}_{\mathrm{c}}\right\rangle_{\beta_{f}}+\beta_{h}^{-1} S\left(\beta_{f}\right)\right)}{d g}\right|_{g=0} & =\left.\frac{d\left(\left\langle\hat{H}_{\mathrm{c}}\right\rangle_{\beta_{f}}-\beta_{h}^{-1} S\left(\beta_{f}\right)\right)}{d \beta_{f}}\right|_{\beta_{f}=\beta_{c}}=-\operatorname{var}\left(\hat{H}_{\mathrm{c}}\right)_{\beta_{c}}+\frac{\beta_{c}}{\beta_{h}} \operatorname{var}\left(\hat{H}_{\mathrm{c}}\right)_{\beta_{c}} \\
& =\frac{\beta_{c}-\beta_{h}}{\beta_{h}} \operatorname{var}\left(\hat{H}_{\mathrm{c}}\right)_{\beta_{c}} .
\end{aligned}
$$

This implies that when fully expanded, Eq. (210) reads as

$$
\begin{aligned}
W_{1}= & n g \frac{\beta_{c}-\beta_{h}}{\beta_{h}} \operatorname{var}\left(\hat{H}_{\mathrm{c}}\right)_{\beta_{c}}+\beta_{h}^{-1} \mathrm{~h}_{2}(\varepsilon)+\Theta(\varepsilon g)+\Theta(\varepsilon) \mathrm{h}_{2}(\varepsilon)+\Theta\left(g \varepsilon^{2}\right)+\Theta\left(\varepsilon^{2}\right) \mathrm{h}_{2}(\varepsilon) \\
& +\Theta\left(g^{2}\right)+\Theta\left(\varepsilon g^{2}\right)+\Theta\left(\varepsilon^{2} g^{2}\right) \\
= & n g \frac{\beta_{c}-\beta_{h}}{\beta_{h}} \operatorname{var}\left(\hat{H}_{\mathrm{c}}\right)_{\beta_{c}}+\beta_{h}^{-1}(-\varepsilon \ln \varepsilon+\varepsilon)+\Theta(\varepsilon g)+\Theta\left(\varepsilon^{2} \ln \varepsilon\right)+\Theta\left(\varepsilon^{2}\right)+\Theta\left(g^{2}\right),
\end{aligned}
$$

where we have used $h_{2}(\varepsilon)=-\varepsilon \ln \varepsilon+\Theta(\varepsilon)$, which follows from finding the power-law expansion of the second term in Eq. (46).

Although Eq. (207) is not defined for $\alpha=1$, we can evaluate it in the limit $\alpha \rightarrow 1$ to see if it coincides with the correct expression of $W_{1}$ (in Eq. (215)) at least for the leading order term (found in square brackets of Eq. (207)). For the leading order term of Eq. (207), we find

$$
\begin{aligned}
\lim _{\alpha \rightarrow 1} \frac{1}{\beta_{h}(\alpha-1)}\left[\alpha n g B_{\alpha}-\varepsilon^{\alpha}+\alpha \varepsilon\right] & =\beta_{h}^{-1}\left[n g \lim _{\alpha \rightarrow 1} \frac{\alpha B_{\alpha}}{\alpha-1}-\lim _{\alpha \rightarrow 1} \frac{\varepsilon^{\alpha}-\alpha \varepsilon}{\alpha-1}\right] \\
& =\beta_{h}^{-1}\left[n g \lim _{\alpha \rightarrow 1} \frac{\alpha B_{\alpha}}{\alpha-1}+(-\varepsilon \ln \varepsilon+\varepsilon)\right], \\
& =n g \frac{\beta_{c}-\beta_{h}}{\beta_{h}} \operatorname{var}\left(\hat{H}_{\mathrm{c}}\right)_{\beta_{c}}+\beta_{h}^{-1}(-\varepsilon \ln \varepsilon+\varepsilon) .
\end{aligned}
$$


The last equality holds because

$$
\begin{aligned}
\lim _{\alpha \rightarrow 1} \frac{\alpha B_{\alpha}}{\alpha-1} & =\lim _{\alpha \rightarrow 1} \frac{d B_{\alpha}}{d \alpha} \\
& =\left(\beta_{c}-\beta_{h}\right) \cdot \operatorname{var}\left(\hat{H}_{\mathrm{c}}\right)_{\beta_{c}},
\end{aligned}
$$

where Eq. (219) is derived from L'Hôspital rule $\left(B_{1}=0\right.$ follows from the definition, see Eq. (188)), and Eq. (220) comes by invoking Lemma 10. Thus noting that Eq. (218) is simply the first two terms in Eq. (220), we conclude that the small $g>0$ and $\varepsilon>0$ expansion of $W_{\alpha}$ for $\alpha \in(0, \infty]$ can be summarized as

$$
\begin{aligned}
& W_{\alpha}= \\
& \begin{cases}\frac{1}{\beta_{h}(\alpha-1)}\left[\alpha n g B_{\alpha}-\varepsilon^{\alpha}+\alpha \varepsilon\right]+\Theta\left(g^{2}\right)+\Theta\left(\varepsilon^{2 \alpha}\right)+\Theta\left(g \varepsilon^{\alpha}\right)+\Theta\left(\varepsilon^{2}\right) & \text { if } \alpha \in(0,1) \cup(1, \infty], \\
\lim _{\alpha \rightarrow 1^{+}} \frac{1}{\beta_{h}(\alpha-1)}\left[\alpha n g B_{\alpha}-\varepsilon^{\alpha}+\alpha \varepsilon\right]+\Theta(\varepsilon g)+\Theta\left(\varepsilon^{2} \ln \varepsilon\right)+\Theta\left(\varepsilon^{2}\right)+\Theta\left(g^{2}\right) & \text { if } \alpha=1 .\end{cases}
\end{aligned}
$$

(C) For $\alpha=0$

We will now investigate the $\alpha=0$ case. This is also particularly important to understand the difference between perfect and near perfect work, since in Section E.1, the impossibility of extracting perfect work arises from evaluating the allowed values of $W_{\text {ext }}$ under the $\alpha=0$ constraint. We show that by allowing $\varepsilon>0, W_{\text {ext }}>0$ is allowed once again. Recall $D_{0}(p \| q)=\lim _{\alpha \rightarrow 0} D_{\alpha}(p \| q)=\sum_{i: p_{i} \neq 0} q_{i}$. Thus from Eq. $(171)$

$$
D_{0}\left(\rho_{\mathrm{W}}^{0} \| \tau_{\mathrm{W}}\right)-D_{0}\left(\rho_{\mathrm{W}}^{1} \| \tau_{\mathrm{W}}\right) \geq n D_{0}\left(\tau_{\beta_{f}} \| \tau_{\beta_{h}}\right)-n D_{0}\left(\tau_{\beta_{c}} \| \tau_{\beta_{h}}\right)=0 .
$$

where the last equality follows from the fact that thermal states have full rank. This inequality is satisfied for any value of $W_{\text {ext }}$, since whenever $\varepsilon>0, \rho_{\mathrm{W}}^{1}$ is a full rank state, and $D_{0}\left(\rho_{\mathrm{W}}^{1} \| \tau_{\mathrm{W}}\right)=0$. Furthermore, $D_{0}\left(\rho_{\mathrm{W}}^{0} \| \tau_{\mathrm{W}}\right) \geq 0$ because all Rényi divergences are non-negative. Therefore, taking into account Eqs. (221) and (223), for quasi-static heat engines which extract near perfect work, we only need to solve

$$
W_{\text {ext }}=\inf _{\alpha>0} W_{\alpha}
$$

where $W_{\alpha}$ is given by Eq. (221).

\section{E.2.4 The choice of $\varepsilon$ determines the infimum to evaluating $W_{\text {ext }}$}

In this section, we will show that the infimum over $\alpha>0$ in Eq. (224) can be simplified to taking the infimum over $\alpha>\bar{\kappa}$ instead, where the parameter $\bar{\kappa}$ determines how quickly $\varepsilon$ goes to 0 w.r.t. the parameter $g$. We define $\kappa$ introduce $\bar{\kappa}$ in Lemma 11, for functions of $\varepsilon(g)$.

Lemma 11. For every continuous function $\varepsilon(g)>0$ satisfying $\lim _{g \rightarrow 0^{+}} \varepsilon(g)=0$, for which the limit $\lim _{g \rightarrow 0^{+}} \varepsilon^{\kappa}(g) / g, \kappa \in \mathbb{R}$ exists, we have that $\exists \bar{\kappa} \in \mathbb{R}_{\geq 0}$ s.t.

$$
\delta(\kappa)=\lim _{g \rightarrow 0^{+}} \frac{\varepsilon^{\kappa}(g)}{g}= \begin{cases}0 & \text { if } \kappa>\bar{\kappa} \\ \sigma \geq 0 & \text { if } \kappa=\bar{\kappa} \\ \infty & \text { if } \kappa<\bar{\kappa}\end{cases}
$$

where $\bar{\kappa}=+\infty$ is allowed (that is to say, $\lim _{g \rightarrow 0^{+}} \frac{\varepsilon^{\kappa}(g)}{g}$ diverges for every $\kappa$ ) and $\sigma=+\infty$ is also allowed.

Proof. The main idea in this proof is to divide the non-negative real line into an infinite sequence of intervals in an iterative process. We specify the ends of these intervals by constructing a sequence $\left\{\kappa_{i}\right\}_{i=1}^{\infty}$, and evaluating $\delta$ at these points. We then prove that according to our construction, there 


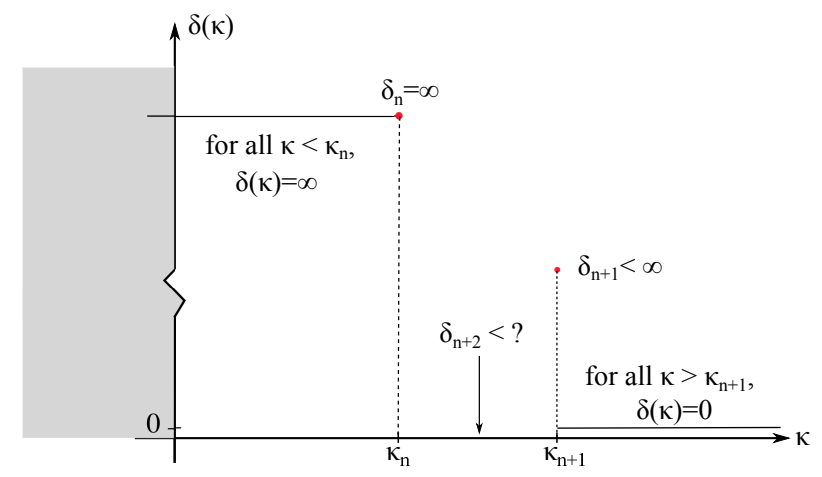

Figure 6: Illustration of the scenario where $\delta\left(\kappa_{n}\right)=\infty$ and $\delta\left(\kappa_{n+1}\right)<\infty$.

are only two possibilities:

1) $\kappa_{i}$ forms a convergent sequence, where the $\operatorname{limit}_{n \rightarrow \infty} \kappa_{n}=\bar{\kappa}$, or

2 ) the ends of these intervals extend to infinity. In this case, $\bar{\kappa}=\infty$. The way to construct this interval is as follows: in the first round, pick some $\kappa_{1}>0$. The corresponding interval is $\left[0, \kappa_{1}\right]$. Evaluate $\delta\left(\kappa_{1}\right)$. If $\delta\left(\kappa_{1}\right)=\infty$, then proceed to look at the interval $\left[\kappa_{1}, \frac{3}{2} \kappa_{1}\right]$. Otherwise if $\delta\left(\kappa_{1}\right)<\infty$, choose $\kappa_{2}=\frac{\kappa_{1}}{2}$ and evaluate $\delta\left(\kappa_{2}\right)$. Depending on whether $\delta\left(\kappa_{2}\right)$ goes to infinity, we pick one of the intervals $\left[0, \kappa_{2}\right]$ or $\left[\kappa_{2}, \kappa_{1}\right]$.

A general expression of choosing $\kappa_{n}$ can be written: during the $n$th round, define the sets $\mathcal{S}_{n}^{(0)}, \mathcal{S}_{n}^{(\infty)}$ such that

$$
\begin{gathered}
\mathcal{S}_{n}^{(0)}=\left\{\kappa_{i} \mid 1 \leq i \leq n \text { and } \delta\left(\kappa_{i}\right)=0\right\} \\
\mathcal{S}_{n}^{(\infty)}=\left\{\kappa_{i} \mid 1 \leq i \leq n \text { and } \delta\left(\kappa_{i}\right)=\infty\right\} .
\end{gathered}
$$

Note that if we find $\delta\left(\kappa_{i}\right)=c \neq 0$ for some finite constant $c$, then our job is finished, i.e. $\bar{\kappa}=\kappa_{i}$ (We prove this later). Subsequently, define for $n \geq 1$,

$$
\kappa_{n}^{(0)}=\min _{\kappa \in \mathcal{S}_{n}^{(0)}} \kappa \quad \text { and } \quad \kappa_{n}^{(\infty)}=\max _{\kappa \in \mathcal{S}_{n}^{(\infty)}} \kappa .
$$

If either sets are empty, we use the convention that the corresponding minimization/maximization equals 0 . Once these quantities are defined, we can choose the next interval by evaluating

$$
\kappa_{n+1}=\kappa_{n}^{(\infty)}+\frac{\left|\kappa_{n}^{(\infty)}-\kappa_{n}^{(0)}\right|}{2} .
$$

In the $n$-th round, the corresponding interval is $\left[\kappa_{n}^{(\infty)}, \kappa_{n+1}\right]$.

Let us now analyze why we can use this scheme to find $\bar{\kappa}$. Firstly, consider the case where $\delta\left(\kappa_{i}\right)$ whenever evaluated, produces infinity. This means that in each round, $\kappa_{n}^{(\infty)}=\kappa_{n}$ increases with $n$ (by the iterative scheme), and $\kappa_{n}^{(0)}=0$ always stays at zero. Note that this scheme has been constructed in a way such that $\lim _{n \rightarrow \infty} \kappa_{n}=\infty$. Indeed, for all $n$, by using Eq. (226),

$$
\kappa_{n+1}=\frac{3}{2} \kappa_{n}=\left(\frac{3}{2}\right)^{2} \kappa_{n-1}=\cdots=\left(\frac{3}{2}\right)^{n} \kappa_{1},
$$

which tends to infinity as $n$ goes to infinity, whenever $\kappa_{1}>0$. Later we will prove a property of the function $\delta$, which combined with this scenario means that $\delta(\kappa)=\infty$ for every $\kappa \geq 0$. Therefore, $\bar{\kappa}=\infty$.

Next, suppose that there exist an $n$-th round, such that $\delta\left(\kappa_{n}\right)=\infty$ and $\delta\left(\kappa_{n+1}\right)<\infty$, as illustrated in Fig 6. Note that the function $\delta(\kappa)$ has a peculiar property, i.e. we know that if $\delta\left(\kappa_{n}\right)=\infty$, then 
for any $\kappa<\kappa_{n}$,

$$
\delta(\kappa)=\lim _{g \rightarrow 0^{+}} \underbrace{\varepsilon^{\kappa-\kappa_{n}}(g)}_{\rightarrow+\infty} \underbrace{\frac{\varepsilon^{\kappa_{n}}(g)}{g}}_{\rightarrow \infty}=+\infty .
$$

On the other hand, if $\delta\left(\kappa_{n+1}\right)=0$, then we know that for any $\kappa>\kappa_{n+1}$,

$$
\delta(\kappa)=\lim _{g \rightarrow 0^{+}} \underbrace{\varepsilon^{\kappa-\kappa_{n+1}}(g)}_{\rightarrow 0} \underbrace{\frac{\varepsilon^{\kappa_{n+1}}(g)}{g}}_{\rightarrow 0}=0 .
$$

Moreover, if $\delta\left(\kappa_{j}\right)=c \neq 0$ for some positive, finite $c$, then following the same arguments, one can easily see that for all $\kappa<\kappa_{j}, \delta(\kappa)=\infty$ and for $\kappa>\kappa_{j}, \delta(\kappa)=0$. In this case we find that $\bar{\kappa}=\kappa_{j}$. These observations are illustrated in Figure 6 for clarity.

One can now evaluate $\kappa_{n+2}$ (which is the midpoint of $\kappa_{n}$ and $\kappa_{n+1}$ ) and its corresponding value of $\delta\left(\kappa_{n+2}\right)$. From this point on, in each iteration we either find $\bar{\kappa}$ exactly (whenever the function $\delta$ when evaluated produces a finite, non-zero number), or the length of the next interval gets halved, and goes to zero in the limit of $n \rightarrow \infty$. This, by Eq. (226), also implies that $\lim _{n \rightarrow \infty} \kappa_{n}^{(\infty)}=\lim _{n \rightarrow \infty} \kappa_{n}^{(0)}$. We also know the following:

1) for all $\kappa<\kappa_{n}^{(\infty)}, \delta(\kappa)=\infty$,

2) for all $\kappa>\kappa_{n}^{(0)}, \delta(\kappa)=0$.

Therefore, we see that $\bar{\kappa}$ exists and $\bar{\kappa}=\lim _{n \rightarrow \infty} \kappa_{n}^{(\infty)}=\lim _{n \rightarrow \infty} \kappa_{n}^{(0)}$. By this we conclude the proof.

To provide some intuition about how $\bar{\kappa}$ compares the rate of convergence $\varepsilon, g \rightarrow 0$, let us look at the following examples:

1) Consider $\varepsilon_{1}(g)=\exp (-1 / g)$. Then $\bar{\kappa}=0$ with $\sigma=\infty$.

2) Consider $\varepsilon_{2}(g)=g \ln g$. Then $\bar{\kappa}=1$ with $\sigma=\infty$.

3) Consider $\varepsilon_{3}(g)=c \cdot g^{1 / k}$ for $k>0$. Then $\bar{\kappa}=k$ with $\sigma=c$.

In the next lemma, we consider the scenario of near perfect work, given in Def. 5, and show that this imposes a finite range of values $\bar{\kappa}$ should take. Given a particular $\bar{\kappa}$, we also show that the minimization of Eq. (224) changes with $\bar{\kappa}$.

Lemma 12. Given any $\varepsilon(g) \in(0,1]$ as a continuous function of $g$, where $g>0$. If $\lim _{g \rightarrow 0^{+}} \varepsilon(g)=0$ and $\lim _{g \rightarrow 0^{+}} \frac{\Delta S}{W_{\text {ext }}}=0$, then the following holds:

1. The quantity $\bar{\kappa}$ (defined in Lemma 11) can only have any value in $\bar{\kappa} \in[0,1]$, where $\lim _{g \rightarrow 0^{+}} \frac{\varepsilon \ln \varepsilon}{g}=$ 0 has to hold if $\bar{\kappa}=1$.

2. The extractable work can be written as

$$
\beta_{h} W_{\text {ext }}=g \cdot\left[\inf _{\alpha \geq \bar{\kappa}} \frac{n \alpha B_{\alpha}}{\alpha-1}+f(g)\right],
$$

where $\lim _{g \rightarrow 0^{+}} f(g)=0$ and $\inf _{\alpha \geq \bar{\kappa}}$ can be exchanged for $\inf _{\alpha>\bar{\kappa}}$ if $\bar{\kappa}=0$.

Proof. Firstly, let us use Eq. (221) to simplify our expression for $W_{\text {ext }}: W_{\text {ext }}=\inf _{\alpha \geq 0} W_{\alpha}$, where

$$
\beta_{h} W_{\alpha}= \begin{cases}g \tilde{W}_{\alpha}+\Theta\left(g^{2}\right)+\Theta\left(\varepsilon^{2 \alpha}\right)+\Theta\left(g \varepsilon^{\alpha}\right)+\Theta\left(\varepsilon^{2}\right) & \text { if } \alpha \in(0,1) \cup(1, \infty] \\ g \tilde{W}_{1}+\Theta(\varepsilon g)+\Theta\left(\varepsilon^{2} \ln \varepsilon\right)+\Theta\left(\varepsilon^{2}\right)+\Theta\left(g^{2}\right) & \text { if } \alpha=1,\end{cases}
$$

and

$$
\tilde{W}_{\alpha}:=\frac{1}{\alpha-1}\left(\alpha n B_{\alpha}+\alpha \frac{\varepsilon}{g}-\frac{\varepsilon^{\alpha}}{g}\right)
$$


and for $\alpha=1$

$$
\tilde{W}_{1}=\lim _{\alpha \rightarrow 1} \tilde{W}_{\alpha}=\left(\lim _{\alpha \rightarrow 1} \frac{\alpha n B_{\alpha}}{\alpha-1}\right)+\frac{\varepsilon}{g}-\frac{\varepsilon}{g} \ln (\varepsilon) .
$$

From now on, the order terms in Eq. (231) can be neglected, since it can be checked that all of them are of higher order compared to the terms we grouped in $\tilde{W}_{\alpha}$, in the limit of vanishing $g$. Even then, we note that due to the complicated form of $W_{\text {ext }}$, it is not straightforward to begin our proof with the assumption $\lim _{g \rightarrow 0^{+}} \frac{\Delta S}{W_{\text {ext }}}=0$.

Instead, we begin by noting that given a function $\varepsilon(g)$ that satisfies the conditions of the above lemma, then one can invoke Lemma 11, and therefore there exists a $\bar{\kappa} \in \mathbb{R}_{\geq 0}$ such that Eq. (225) holds. We then, for all possible $\kappa \in \mathbb{R}_{\geq 0}$, evaluate all $\tilde{W}_{\alpha}$ to take the infimum and obtain $W_{\text {ext }}$. Given $W_{\text {ext }}$, we then evaluate the quantity $\lim _{g \rightarrow 0^{+}} \frac{\Delta S}{W_{\text {ext }}}=0$.

The value of $\bar{\kappa}$ determines how the limits of quantities like $\frac{\varepsilon}{g}, \frac{\varepsilon^{\alpha}}{g}$ behave. Therefore, we need to split the analysis into three different regimes: $\bar{\kappa} \in[0,1), \bar{\kappa}=1, \bar{\kappa} \in(1, \infty)$.

1) For $\bar{\kappa} \in[0,1)$

For this case, we know the following limits:

A. $\lim _{g \rightarrow 0^{+}} \frac{\varepsilon}{g}=0$.

B. For $\alpha<\bar{\kappa}, \lim _{g \rightarrow 0^{+}} \frac{\varepsilon^{\alpha}}{g}=\infty$.

C. For $\alpha=\bar{\kappa}, \lim _{g \rightarrow 0^{+}} \frac{\varepsilon^{\alpha}}{g}=\sigma \geq 0$.

D. For $\alpha>\bar{\kappa}, \lim _{g \rightarrow 0^{+}} \frac{\varepsilon^{\alpha}}{g}=0$.

E. Note that $\exists k_{1}>\bar{\kappa}$ such that $1-k_{1}>0$. Thus $\lim _{g \rightarrow 0^{+}} \frac{\varepsilon}{g} \ln \varepsilon=\lim _{g \rightarrow 0^{+}} \frac{\varepsilon^{k_{1}}}{g} \varepsilon^{1-k_{1}} \ln \varepsilon=0$

Therefore, by using Eq. (232) and (233) (for $\alpha=1$ separately) we have

$$
\tilde{W}_{\alpha}= \begin{cases}+\infty & \text { if } \alpha \in[0, \bar{\kappa}) \\ \frac{\alpha n B_{\alpha}}{\alpha-1}+\frac{1}{\bar{\kappa}-1}\left(\bar{\kappa} \frac{\varepsilon}{g}-\frac{\varepsilon^{\bar{\kappa}}}{g}\right)=\frac{\alpha n B_{\alpha}}{\alpha-1}+\frac{\sigma}{|\bar{\kappa}-1|}+\Theta\left(\frac{\varepsilon}{g}\right) \bar{\kappa} & \text { if } \alpha=\bar{\kappa} \\ \frac{\alpha n B_{\alpha}}{\alpha-1}+\Theta\left(\frac{\varepsilon^{\alpha}}{g}\right) & \text { if } \alpha \in(\bar{\kappa}, 1) \\ \frac{\alpha n B_{\alpha}}{\alpha-1}+\Theta\left(\frac{\varepsilon}{g}\right) & \text { if } \alpha \in(1, \infty] \\ \lim _{\alpha \rightarrow 1} \frac{\alpha n B_{\alpha}}{\alpha-1}+\Theta\left(\frac{\varepsilon \ln \varepsilon}{g}\right) & \text { if } \alpha=1,\end{cases}
$$

where the expression in Eq.(234) has been written as a leading order term, plus higher order terms that vanish in the limit $g \rightarrow 0{ }^{10}$. In the second line we have used $\left(\bar{\kappa} \varepsilon-\varepsilon^{\bar{\kappa}}\right) /(\bar{\kappa}-1)=\left|\left(\bar{\kappa} \varepsilon-\varepsilon^{\bar{\kappa}}\right) /(\bar{\kappa}-1)\right|$ as $\varepsilon \rightarrow 0^{+}$for $\bar{\kappa} \in[0,1)$.

Therefore, we conclude that for $\bar{\kappa} \in[0,1)$ and any $\sigma \geq 0$, due to continuity in $\alpha$ of $\frac{\alpha n B_{\alpha}}{\alpha-1}$,

$$
\beta_{h} W_{\text {ext }}=\beta_{h} \inf _{\alpha>0} W_{\alpha}=g \cdot\left[\inf _{\alpha \geq \bar{\kappa}} \frac{\alpha n B_{\alpha}}{\alpha-1}+\Theta(f(g))\right],
$$

where $f$ satisfies $\lim _{g \rightarrow 0^{+}} f(g)=0$ in the expression of Eq. (234). Note that if $\bar{\kappa}=0$, then $\inf _{\alpha \geq \bar{\kappa}}$ can be exchanged for $\inf _{\alpha>\bar{\kappa}}$ since in Eq. (224) the point $\alpha=0$ was already excluded.

We can now calculate $\lim _{g \rightarrow 0^{+}} \frac{\Delta S}{W_{\text {ext }}}$ for $\bar{\kappa} \in[0,1)$ and any $\sigma \geq 0$ :

$$
\lim _{g \rightarrow 0^{+}} \frac{\Delta S}{W_{\text {ext }}}=\lim _{g \rightarrow 0^{+}} \frac{-\varepsilon \ln \varepsilon-(1-\varepsilon) \ln (1-\varepsilon)}{\left(\inf _{\alpha \geq \bar{\kappa}} \frac{\alpha n B_{\alpha}}{\alpha-1}\right) g}=\lim _{g \rightarrow 0^{+}} \frac{1}{\inf _{\alpha \geq \bar{\kappa}} \frac{\alpha n B_{\alpha}}{\alpha-1}}(\underbrace{-\frac{\varepsilon \ln \varepsilon}{g}}_{\rightarrow 0 \text { (Item E) }}-\underbrace{\frac{\varepsilon+\Theta\left(\varepsilon^{2}\right)}{g}}_{\rightarrow 0 \text { (Item A) }})=0,
$$

${ }^{10}$ In Eq. (234), the interval $[0,0)$ is taken to be the empty set (this is relevant for the case $\bar{\kappa}=0$ ). 
where we have assumed that

$$
\inf _{\alpha \geq \bar{\kappa}} \frac{\alpha n B_{\alpha}}{\alpha-1}>0
$$

As we will see later (see Eq. (252)), Eq. (237) holds if $\bar{\kappa}>0$. However, if $\alpha=0$

$$
\frac{\alpha n B_{\alpha}}{\alpha-1}=0,
$$

and we need to use Eq. (234) for the case $\alpha=\bar{\kappa}=0$. The relevant line in Eq. (234) is the 2nd line, from which we have $\tilde{W}_{0}=\sigma=\varepsilon^{\bar{\kappa}} / g=1 / g$. which tends to $+\infty$ as $g \rightarrow 0^{+}$. Alternatively, as already mentioned, this result is also clear since $W_{\alpha}$ for $\alpha=0$ is infinite since it expresses the rank condition for state transitions which is always satisfied regardless of how much work is being extracted. Hence

$$
\lim _{g \rightarrow 0^{+}} \frac{\Delta S}{W_{\text {ext }}}=0
$$

in this case also. Thus from Eqs. (238) and (239), we conclude that Eqs. (235) and (236) are still valid when $\bar{\kappa}=0$. To summarize, so far we have proven that whenever $\bar{\kappa} \in[0,1)$, Eq. (230) holds for some $f(g)$ which vanishes as $g$ tends to zero, and furthermore $\lim _{g \rightarrow 0^{+}} \frac{\Delta S}{W_{\mathrm{ext}}}=0$.

2) For $\bar{\kappa} \in(1, \infty)$

In this regime, like the previous analysis, we can list out the following limits:

A. $\lim _{g \rightarrow 0^{+}} \frac{\varepsilon}{g}=0$.

B. By definition of $\bar{\kappa}$, for $\alpha<1, \lim _{g \rightarrow 0^{+}} \frac{\varepsilon^{\alpha}}{g}=\infty$.

C. $\lim _{g \rightarrow 0^{+}} \frac{\varepsilon \ln \varepsilon}{g}=\infty$ since both $\frac{\varepsilon}{g}$ and $\ln \varepsilon$ goes to infinity as $g \rightarrow 0$.

Therefore, by using Eq. (232) and (233) (for $\alpha=1$ separately) we have

$$
\tilde{W}_{\alpha}= \begin{cases}\frac{1}{g} \cdot \frac{1}{1-\alpha}\left[\varepsilon^{\alpha}+\Theta(\varepsilon)+\Theta(g)\right] & \text { if } \alpha \in[0,1) \\ \frac{1}{g} \cdot[-\varepsilon \ln \varepsilon+\Theta(\varepsilon)+\Theta(g)] & \text { if } \alpha=1 \\ \frac{1}{g} \cdot \frac{1}{\alpha-1}\left[\alpha \varepsilon+\Theta\left(\varepsilon^{\alpha}\right)+\Theta(g)\right] & \text { if } \alpha \in(1, \infty] .\end{cases}
$$

Note that for all of these expressions in Eq. (240), $\tilde{W}_{\alpha} \rightarrow \infty$ as $g \rightarrow 0^{+}$. Next we want to calculate $W_{\text {ext }}$, which is the infimum of $W_{\alpha}$, taken over all $\alpha \geq 0$. Note that in the limit of vanishing $g, \varepsilon$ also goes to zero. Therefore from Eq. (240), we see that the equation for $g \tilde{W}_{\alpha}$ which vanishes most quickly in the limit $g \rightarrow 0$ happens when $\alpha \in(1, \infty]$. Therefore, we conclude that for $\bar{\kappa} \in(1, \infty)$ and any $\sigma \geq 0$

$$
\beta_{h} W_{\mathrm{ext}}=\beta_{h} \inf _{\alpha \geq 1} W_{\alpha}=g \cdot\left[\inf _{\alpha \geq 1} \frac{\alpha}{\alpha-1} \frac{\varepsilon}{g}+\Theta(f(g))\right]=\varepsilon+g \cdot \Theta(f(g))
$$

We can now calculate $\lim _{g \rightarrow 0^{+}} \frac{\Delta S}{W}$ for $\bar{\kappa} \in(1, \infty)$ and any $\sigma \geq 0$ :

$$
\lim _{g \rightarrow 0^{+}} \frac{\Delta S}{W}=\lim _{g \rightarrow 0^{+}} \frac{-\varepsilon \ln \varepsilon-(1-\varepsilon) \ln (1-\varepsilon)}{\varepsilon}=\lim _{g \rightarrow 0^{+}} \underbrace{-\frac{\varepsilon \ln \varepsilon}{\varepsilon}}_{\rightarrow \infty}-\underbrace{\frac{\varepsilon+\Theta\left(\varepsilon^{2}\right)}{\varepsilon}}_{\rightarrow 1}=+\infty .
$$

From this, we note that the whole regime of $\bar{\kappa} \in(1, \infty)$ does not contain any cases corresponding to our condition of interest: $\lim _{g \rightarrow 0^{+}} \frac{\Delta S}{W_{\text {ext }}}=0$ never holds.

3) For $\bar{\kappa}=1$

Similar to the first two cases, we again list out the relevant limits:

A. $\lim _{g \rightarrow 0^{+}} \frac{\varepsilon}{g}=\sigma$ for some $\sigma \geq 0$.

B. For $\alpha<1, \lim _{g \rightarrow 0^{+}} \frac{\varepsilon^{\alpha}}{g}=\infty$. 
C. For $\alpha>1, \lim _{g \rightarrow 0^{+}} \frac{\varepsilon^{\alpha}}{g}=0$.

Therefore, by using Eq. (232) and (233) (for $\alpha=1$ separately) we have

$$
\tilde{W}_{\alpha}= \begin{cases}\frac{1}{g} \cdot \frac{1}{1-\alpha}\left[\varepsilon^{\alpha}+\Theta(\varepsilon)+\Theta(g)\right] & \text { if } \alpha \in[0,1) \\ \frac{1}{g} \cdot[-\varepsilon \ln \varepsilon+\Theta(\varepsilon)+\Theta(g)] & \text { if } \alpha=1 \wedge \sigma>0 \\ n \lim _{\alpha \rightarrow 1} \frac{\alpha B_{\alpha}}{\alpha-1}-\frac{\varepsilon \ln \varepsilon}{g} \geq n \lim _{\alpha \rightarrow 1} \frac{\alpha B_{\alpha}}{\alpha-1} & \text { if } \alpha=1 \wedge \sigma=0 \\ \frac{1}{\alpha-1}\left[\alpha n B_{\alpha}+\alpha \sigma-\Theta\left(\frac{\varepsilon^{\alpha}}{g}\right)\right] & \text { if } \alpha \in(1, \infty] .\end{cases}
$$

Note that for $\alpha \in[0,1)$ and the case $\alpha=1 \wedge \sigma>0, \tilde{W}_{\alpha}$ tends to infinity, while for the other cases $\tilde{W}_{\alpha}$ is finite.

Therefore, we can conclude that for $\bar{\kappa}=1$,

$$
\beta_{h} W_{\mathrm{ext}}=g \cdot\left[\left(\inf _{\alpha \geq 1} \frac{\alpha}{\alpha-1}\left(n B_{\alpha}+\sigma\right)\right)+\Theta(f(g))\right],
$$

where $f(g)=\frac{\varepsilon^{\alpha}}{g}$ vanishes as $g$ tends to zero.

Now, we evaluate the limit $\lim _{g \rightarrow 0^{+}} \frac{\Delta S}{W}$ for $\bar{\kappa}=1$ and any $\sigma \geq 0$ :

$$
\lim _{g \rightarrow 0^{+}} \frac{\Delta S}{W}=\lim _{g \rightarrow 0^{+}} \frac{-\varepsilon \ln \varepsilon-(1-\varepsilon) \ln (1-\varepsilon)}{\left(\inf _{\alpha \geq 1} \frac{1}{\alpha-1}\left(\alpha n F_{\alpha}+\alpha \sigma\right)\right) g}=\lim _{g \rightarrow 0^{+}} \frac{-\varepsilon \ln \varepsilon}{c \cdot g}-\underbrace{\frac{\varepsilon+\Theta\left(\varepsilon^{2}\right)}{c \cdot g}}_{\rightarrow 0} .
$$

This limit of interest can be zero if and only if $\lim _{g \rightarrow 0^{+}} \frac{\varepsilon \ln \varepsilon}{g}=0$.

We have calculated the limits $\lim _{g \rightarrow 0^{+}} \Delta S / W_{\text {ext }}$ to leading order in $g$ for all functions $\varepsilon(g)>0$ satisfying $\lim _{g \rightarrow 0^{+}} \varepsilon=0$. These are found in Eqs. (236), (242), and (245). We have found that $\lim _{g \rightarrow 0^{+}} \Delta S / W_{\text {ext }}=0$ occurs only in two cases:

i) $\bar{\kappa} \in[0,1)$, and

ii) $\bar{\kappa}=1$ and $\lim _{g \rightarrow 0^{+}} \frac{\varepsilon \ln \varepsilon}{g}=0$.

The amount of work, $W_{\text {ext }}$ is found in Eq. (235) and (244) respectively. Indeed, they take the form of Eq. (230), for different functions $f(g)$. With this, we conclude the proof of the lemma.

\section{E.2.5 Solving the infimum for $W_{\text {ext }}$}

We have seen in Lemma 12 that the function $\frac{\alpha B_{\alpha}}{\alpha-1}$ corresponds to the largest order term in $W_{\text {ext }}$ w.r.t. small $\mathrm{g}$ (quasi-static heat engine). Our next objective is to find the infimum of $\frac{\alpha B_{\alpha}}{\alpha-1}$ over $\alpha \in[\bar{\kappa}, \infty]$ appearing in Eq. (230). Such an infimum is is not easy to evaluate, but whenever the cold bath consists of multiple identical qubits, we show that the derivative $\frac{d}{d \alpha} \frac{\alpha B_{\alpha}}{\alpha-1}$ has some nice properties. Roughly speaking, we show that this derivative does not have many roots, which in turn means that $\frac{\alpha B_{\alpha}}{\alpha-1}$ does not have many turning points. We have used this to prove in Lemma 14 that the infimum is either obtained at $\alpha=\bar{\kappa}$ or $\alpha \rightarrow \infty$.

The derivative of $\frac{\alpha B_{\alpha}}{\alpha-1}$ w.r.t. $\alpha$ is given by

$$
\frac{d}{d \alpha} \frac{\alpha B_{\alpha}}{\alpha-1}=\frac{B_{\alpha}}{\alpha-1}+\alpha \frac{B_{\alpha}^{\prime}}{\alpha-1}-\frac{\alpha B_{\alpha}}{(\alpha-1)^{2}}=\frac{B_{\alpha}^{\prime}}{(\alpha-1)^{2}}\left[\alpha(\alpha-1)-\frac{B_{\alpha}}{B_{\alpha}^{\prime}}\right]=\frac{B_{\alpha}^{\prime}}{(\alpha-1)^{2}} G(\alpha),
$$

where

$$
G(\alpha):=\alpha(\alpha-1)-\frac{B_{\alpha}}{B_{\alpha}^{\prime}}
$$


Now, we shall evaluate the quantities $B_{\alpha}, B_{\alpha}^{\prime}$, and $\frac{d}{d \alpha} \frac{B_{\alpha}}{B_{\alpha}^{\prime}}$ for the case of qubits (see Assumption (A.5)), where the energy levels are $\{0, E\}$. By using Eq. (180), we evaluate the quantity $B_{\alpha}$ defined by Eq. (189) to obtain a simple expression:

$$
\begin{aligned}
B_{\alpha} & =E \cdot \frac{e^{-\beta_{c} E}}{1+e^{-\beta_{c} E}}-E \cdot \frac{e^{-\alpha \beta_{c} E} e^{-(1-\alpha) \beta_{h} E}}{1+e^{-\alpha \beta_{c} E} e^{-(1-\alpha) \beta_{h} E}} \\
& =E \cdot \frac{1}{1+e^{\beta_{c} E}}-E \cdot \frac{e^{\alpha \beta_{h} E}}{e^{\alpha \beta_{h} E}+e^{\left(\beta_{h}+\alpha \beta_{c}\right) E}} \\
& =\frac{E}{1+e^{\beta_{c} E}} \cdot\left[1-\frac{e^{\alpha \beta_{h} E}\left(1+e^{\beta_{c} E}\right)}{e^{\alpha \beta_{h} E}+e^{\left(\beta_{h}+\alpha \beta_{c}\right) E}}\right] \\
& =\frac{E}{1+e^{\beta_{c} E}} \cdot \frac{e^{\left(\beta_{h}+\alpha \beta_{c}\right) E}-e^{\left(\beta_{c}+\alpha \beta_{h}\right) E}}{e^{\alpha \beta_{h} E}+e^{\left(\beta_{h}+\alpha \beta_{c}\right) E}} .
\end{aligned}
$$

We note that Eq. (251) is zero only if $\alpha=1$, and thus for $\alpha \neq 1, \alpha B_{\alpha} /(\alpha-1) \neq 0$. From Eq. (220), we know that $\lim _{\alpha \rightarrow 1} \alpha B_{\alpha} /(\alpha-1)>0$, thus due to continuity,

$$
\frac{\alpha B_{\alpha}}{\alpha-1}>0 \quad \forall \alpha>0
$$

We also derive the first derivative of $B_{\alpha}$ w.r.t. $\alpha$ for the special case of qubits:

$$
B_{\alpha}^{\prime}=\frac{d B_{\alpha}}{d \alpha}=\frac{E^{2}\left(\beta_{c}-\beta_{h}\right)}{\left[e^{\alpha \beta_{h} E}+e^{\left(\beta_{h}+\alpha \beta_{c}\right) E}\right]^{2}} \cdot e^{\left(\beta_{h}+\alpha \beta_{c}+\alpha \beta_{h}\right) E} .
$$

Note that since $\beta_{c}>\beta_{h}$ by definition, therefore whenever $E>0$, then $B_{\alpha}^{\prime}>0$ always holds. By further algebraic manipulation, we compute the first derivative of the function

$$
\frac{d}{d \alpha} \frac{B_{\alpha}}{B_{\alpha}^{\prime}}=\frac{\cosh \left[w\left(\beta_{c}, \beta_{h}, \alpha\right) E\right]}{\cosh \left(\beta_{c} E / 2\right)}
$$

where $w\left(\beta_{c}, \beta_{h}, \alpha\right)=\left(\beta_{c}-\beta_{h}\right) \alpha+\beta_{h}-\frac{\beta_{c}}{2}$.

We have written Eq. (246) in this form, since for the special case of qubits, namely Eq. (253), $B_{\alpha}^{\prime}>0$ is always true. Therefore, looking at the function $G(\alpha)$ whether it is positive or negative) will tell us whether $\frac{\alpha B_{\alpha}}{\alpha-1}$ (and therefore $W_{\alpha}$ ) is increasing or decreasing in a particular interval.

In Lemma 13, we identify the conditions on the energy spacing $E$ such that several different properties of $G(\alpha)$ hold.

Lemma 13. Consider $G(\alpha)=\alpha(\alpha-1)-\frac{B_{\alpha}}{B_{\alpha}^{\prime}}$, where $B_{\alpha}, B_{\alpha}^{\prime}$ is defined in Eq. (251) and (253). Then the following holds:

1) If $E\left(\beta_{c}-\beta_{h}\right) \tanh \left(\beta_{c} E / 2\right)>2$,

$$
\exists 0<\tau<1 \text { s.t. } \quad G(\alpha)<0 \quad \forall \alpha \in(\tau, 1) \cup(1, \infty)
$$

2) If $E\left(\beta_{c}-\beta_{h}\right) \tanh \left(\beta_{c} E / 2\right)<2$,

$$
\begin{array}{lll}
\exists \underline{\alpha}>1 \text { s.t. } & G(\alpha)>0 \quad \forall \alpha \in(0,1) \cup(1, \underline{\alpha}) \\
& G(\alpha)<0 \quad \forall \alpha \in(\underline{\alpha}, \infty) .
\end{array}
$$

3) If $E\left(\beta_{c}-\beta_{h}\right) \tanh \left(\beta_{c} E / 2\right)=2$,

$$
\begin{array}{ll}
G(\alpha)>0 & \forall \alpha \in(0,1) \\
G(\alpha)<0 & \forall \alpha \in(1, \infty) .
\end{array}
$$


Proof. First we note that since $B_{1}=0$, therefore $G(1)=0$. Let us also compute the derivative of $G(\alpha)$ w.r.t. $\alpha$ :

$$
G^{\prime}(\alpha)=2 \alpha-1-\frac{\cosh \left(\left(-\beta_{c} / 2+\beta_{h}+\left(\beta_{c}-\beta_{h}\right) \alpha\right) E\right)}{\cosh \left(\beta_{c} E / 2\right)} .
$$

Before we continue, there are several properties of the function $G^{\prime}(\alpha)$ which we shall make use of. Firstly, note that $G^{\prime}(1)=0$, in other words, $G^{\prime}$ has a root at $\alpha=1$. Also, $G^{\prime}(\infty)=-\infty$ for any value of $E>0, \beta_{h}>0, \beta_{c}>\beta_{h}{ }^{11}$. Also, since $2 \alpha-1$ is linear (and hence both convex and concave), while the - cosh function is strictly concave ${ }^{12}$, therefore the function $G^{\prime}(\alpha)$ is strictly concave. This implies that the second derivative $G^{\prime \prime}(\alpha)=\frac{d^{2} G(\alpha)}{d \alpha^{2}}$ is strictly decreasing w.r.t. $\alpha$.

The properties of $G^{\prime}(\alpha)$ indicate that we can fully analyze the function by considering 3 different cases:

1. $G^{\prime}$ has two roots at $\alpha=\{\underline{a}, 1\}$, wherewhere $\underline{a} \in(-\infty, 1)$. This corresponds to the case $G^{\prime \prime}(1)<0$.

2. $G^{\prime}$ has two roots at $\alpha=\{1, \bar{a}\}$, where $\bar{a} \in(1, \infty)$. This corresponds to the case $G^{\prime \prime}(1)>0$.

3. $G^{\prime}$ has a single root at $\alpha=1$. This corresponds to the case $G^{\prime \prime}(1)=0$.

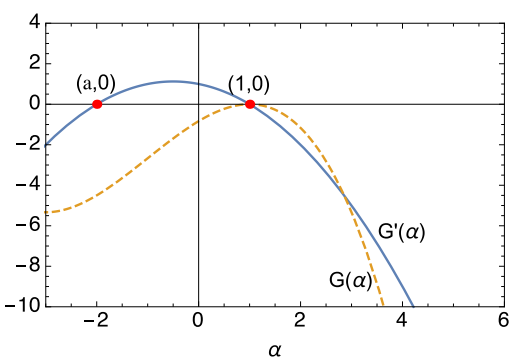

Figure 7: $G^{\prime \prime}(1)<0$.

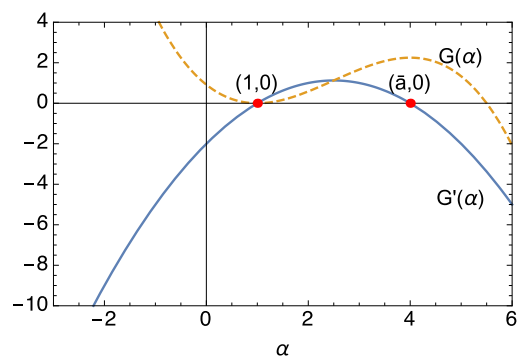

Figure 8: $G^{\prime \prime}(1)>0$.

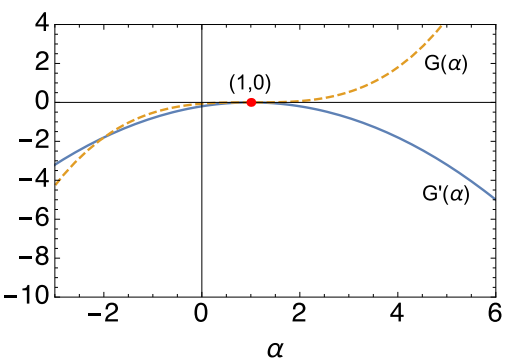

Figure 9: $G^{\prime \prime}(1)=0$.

Figure 10: A convex function $G^{\prime}(\alpha)$ and its corresponding $G(\alpha)$, for different values of $G^{\prime \prime}(\alpha)$.

We shall now consider these cases one by one. Suppose that

$$
G^{\prime \prime}(1)=\left.G^{\prime \prime}(\alpha)\right|_{\alpha=1}=2-\left(\beta_{c}-\beta_{h}\right) E \tanh \left(\frac{\beta_{c} E}{2}\right)<0,
$$

then $G^{\prime \prime}(\alpha)<0$ for all $\alpha \in(1, \infty)$. Note that Eq. (259) corresponds to the first condition in the lemma stated above.

This information about the second derivative $G^{\prime \prime}(\alpha)$ now allows us to conclude the following about $G(\alpha)$ :

1. If for all $\alpha \in(1, \infty), G^{\prime \prime}(\alpha)<0$, then we know that $G^{\prime}(\alpha)<0$ holds for all $\alpha \in(1, \infty)$ too. Furthermore, this implies that $G(\alpha)$ is monotonically decreasing in the interval $(1, \infty)$ and therefore, $G(\alpha)<0$ for all $\alpha \in(1, \infty)$.

2. $G^{\prime \prime}(1)<0$ also implies that there exists an interval $(\tau, 1)$ such that $G^{\prime}(1)>0$ (See Fig. 7). And since $G(1)=0$, this implies that within the interval $(\tau, 1), G(\alpha)<0$.

With this, we prove the first statement of the lemma.

Let us now analyze the second case, where $G^{\prime \prime}(1)>0$. This implies that $G^{\prime}(\alpha)>0$ at least for some interval $\alpha \in(1, \bar{a})$, then $G^{\prime}(\alpha)$ changes sign exactly once at $\alpha=\bar{a}$, and goes to $-\infty$. (Refer to Fig. 8).

\footnotetext{
${ }^{11}$ This is due to the fact that $2 \alpha$ increases linearly w.r.t. $\alpha$, while the cosh term increases exponentially.

${ }^{12}$ To be more precise; due to the concavity of $f(x)=-a \cosh (b+x c)$ for $a>0$. This follows from the strict concavity of the cosh function, the invariancy of strict concavity under an affine transformation and the invariancy of strict concavity under multiplication by a positive constant.
} 
Also, recall that in the limit of $\alpha \rightarrow \infty, G$ also goes to $-\infty$. Therefore, we conclude that there exists some $\bar{\alpha}$ such that

$$
G(\alpha) \begin{cases}>0 & \alpha \in(1, \bar{\alpha}) \\ <0 & \alpha \in(\bar{\alpha}, \infty)\end{cases}
$$

With this, we prove the second statement of the lemma.

Finally, we look at the case where $G^{\prime \prime}(1)=0$, and make the following observations:

1. Since the function $G^{\prime}(\alpha)$ is concave, and since $G^{\prime \prime}(1)=0$ implies that $\alpha=1$ is an extremum point for the function $G^{\prime}(\alpha)$, we know that it must also be the global maximum. Therefore, we know that for any $\alpha \neq 1, G^{\prime}(\alpha)<0$.

2. Since for the interval $\alpha \in(-\infty, 1), G^{\prime}(\alpha)<0$ and we know that $G(1)=0$, therefore we can deduce that for any $\alpha \in(-\infty, 1), G(\alpha)>0$.

3. Since for the interval $\alpha \in(1, \infty), G^{\prime}(\alpha)<0$ and we know that $G(1)=0$, therefore we can deduce that for any $\alpha \in(1, \infty), G(\alpha)<0$.

With this, we prove the final statement of the lemma, and complete our proof.

To summarize, in Lemma 13 we have identified conditions involving the energy gap of $\hat{H}_{\mathrm{c}}$, and the temperatures $\beta_{h}, \beta_{c}$. Depending on whether these conditions are satisfied, we can describe the positivity/negativity of $G(\alpha)$ for different regimes of $\alpha$. Comparing these different scenarios, we prove in Lemma 14 that for a quasi-static heat engine, the minimum of $\inf _{\alpha \geq \bar{\kappa}} \frac{\alpha B_{\alpha}}{\alpha-1}$ is obtained only either at $\alpha=\bar{\kappa}$ or $\alpha=\infty$.

Lemma 14. There exists some $0 \leq \nu<1$ such that $\forall \kappa$ satisfying $\nu<\kappa<1$, the following infimum is obtained at one of two points

$$
\inf _{\alpha \geq \kappa} \frac{\alpha B_{\alpha}}{\alpha-1}=\inf \left\{\lim _{\alpha \rightarrow \kappa} \frac{\alpha B_{\alpha}}{\alpha-1}, \lim _{\alpha \rightarrow \infty} \frac{\alpha B_{\alpha}}{\alpha-1}\right\}<\lim _{\alpha \rightarrow \beta} \frac{\alpha B_{\alpha}}{\alpha-1} \quad \forall \beta \in(\kappa, \infty),
$$

where $B_{\alpha}$ is defined in Eq. (251). Furthermore, if $E\left(\beta_{c}-\beta_{h}\right) \tanh \left(\beta_{c} E / 2\right) \leq 2$, then we can set $\nu=0$.

Proof. 1. If

$$
\frac{d}{d \alpha} \frac{\alpha B_{\alpha}}{\alpha-1}\left\{\begin{array}{l}
>0 \forall \alpha \in(0,1) \cup(1, \bar{\alpha}) \text { for some } \bar{\alpha} \geq 1 \\
<0 \forall \alpha \in(\bar{\alpha}, \infty) .
\end{array}\right.
$$

then $\forall \kappa \in(0,1)$,

$$
\inf _{\alpha \geq \kappa} \frac{\alpha B_{\alpha}}{\alpha-1}=\inf \left\{\lim _{\alpha \rightarrow \kappa} \frac{\alpha B_{\alpha}}{\alpha-1}, \lim _{\alpha \rightarrow \infty} \frac{\alpha B_{\alpha}}{\alpha-1}\right\}<\lim _{\alpha \rightarrow \beta} \frac{\alpha B_{\alpha}}{\alpha-1} \quad \forall \beta \in(\kappa, \infty) .
$$

Recall from Eq. (246) that

$$
\frac{d}{d \alpha} \frac{\alpha B_{\alpha}}{\alpha-1}=\frac{B_{\alpha}^{\prime}}{(\alpha-1)^{2}} G(\alpha)
$$

where $B_{\alpha}^{\prime}>0$, and we have derived some properties of $G(\alpha)$ in Lemma 13. In this proof, we apply Lemma 13 directly to consider the three scenarios detailed in Lemma 13.

First, consider the first statement of Lemma 13. If $E\left(\beta_{c}-\beta_{h}\right) \tanh \left(\beta_{c} E / 2\right)>2$, then $\exists 0<t<1$ s.t.

$$
\frac{d}{d \alpha} \frac{\alpha B_{\alpha}}{\alpha-1}<0 \forall \alpha \in(t, 1) \cup(1, \infty)
$$


then by continuity of $\frac{\alpha B_{\alpha}}{\alpha-1}$ in $\alpha$, we conclude that $\forall \kappa$ satisfying $t<\kappa<1$

$$
\inf _{\alpha \geq \kappa} \frac{\alpha B_{\alpha}}{\alpha-1}=\lim _{\alpha \rightarrow \infty} \frac{\alpha B_{\alpha}}{\alpha-1}=\inf \left\{\lim _{\alpha \rightarrow \kappa} \frac{\alpha B_{\alpha}}{\alpha-1}, \lim _{\alpha \rightarrow \infty} \frac{\alpha B_{\alpha}}{\alpha-1}\right\}<\lim _{\alpha \rightarrow \beta} \frac{\alpha B_{\alpha}}{\alpha-1} \quad \forall \beta \in(\kappa, \infty) .
$$

Next, consider the second and third statements of Lemma 13 jointly, where $E\left(\beta_{c}-\beta_{h}\right) \tanh \left(\beta_{c} E / 2\right)$ $\leq 2$. Note that both statements proved in Lemma 13 (namely, Eq. (256) and (257)) can be rewritten as the fact that there exists $\bar{\alpha} \geq 1$ s.t.

$$
\frac{d}{d \alpha} \frac{\alpha B_{\alpha}}{\alpha-1} \begin{cases}>0 & \text { for } \alpha \in(0,1) \cup(1, \bar{\alpha}) \\ <0 & \text { for } \alpha \in(\bar{\alpha}, \infty)\end{cases}
$$

In fact, the third statement is simply a special case of the second, where $\bar{\alpha}=1$. If Eq. (267) holds, then $\forall \kappa \in(0,1)$,

$$
\inf _{\alpha \geq \kappa} \frac{\alpha B_{\alpha}}{\alpha-1}=\inf \left\{\lim _{\alpha \rightarrow \kappa} \frac{\alpha B_{\alpha}}{\alpha-1}, \lim _{\alpha \rightarrow \infty} \frac{\alpha B_{\alpha}}{\alpha-1}\right\}<\lim _{\alpha \rightarrow \beta} \frac{\alpha B_{\alpha}}{\alpha-1} \quad \forall \beta \in(\kappa, \infty) .
$$

By setting $\tau=0$, we see that the statement of Lemma 14 is achieved.

Therefore, since we have analyzed all three cases stated in Lemma 13, we conclude that there always exists $\nu \in[0,1)$ such that Eq. (261) will always be satisfied $\forall \kappa \in(\nu, 1)$.

\section{E.2.6 Main results: evaluating the efficiency}

In this section, we derive the efficiency of quasi-static heat engines in the nano /quantum regime. We first need to define the quantity

$$
\Omega:=\min _{i \in\{1, \ldots, n\}} \frac{\bar{E}_{i}\left(\beta_{c}-\beta_{h}\right)}{1+e^{-\beta_{c} \bar{E}_{i}}},
$$

where recall that $\bar{E}_{i}$ is the energy gap of the cold bath qubits, as described in Eq (180) and the sentence right after it. Recall that $n$ denotes the number of qubits in the cold bath, where $n \in \mathbb{Z}^{+}$is any positive integer. Before stating the maximum efficiency, we will derive the efficiency as a function of $\bar{\kappa}$ defined in Lemma 11 (recall that this parameter is determined by the choice of $\varepsilon$ ). For simplicity, we will still consider the special case where $\bar{E}_{i}=E$ for all $i$ in Lemma 15, (i.e. all qubits of the cold bath are identical). Lemma 15 shows us that under the condition of extracting near perfect work, one can choose $\varepsilon$ (and therefore $\bar{\kappa}$ ) such that a certain maximum efficiency value is achieved. The closer $\bar{\kappa}$ is to unity, the slower $\lim _{g \rightarrow 0^{+}} \Delta S / W$ converges to zero, and also the closer the efficiency is to the Carnot efficiency.

Using this lemma, we prove the achievability of the Carnot efficiency which depends on $\Omega$. This is the main result of our work, which is stated in Theorem 2.

Lemma 15 (Quasi-static efficiencies as a function of $\bar{\kappa}$ ). For any $n \in \mathbb{Z}^{+}$number of qubits, consider quasi-static heat engines (Def. 6) as a function of $\bar{\kappa}$ (defined in Lemma 11) which extract near perfect work (Def. 5). For any $\kappa \in(0, \infty) \backslash\{1\}$, define

$$
\gamma(\kappa):=\frac{\kappa B_{\kappa}}{\kappa-1}
$$

where $B_{\kappa}$ is defined in Eq. (251), while $\gamma(1)$ and $\gamma(\infty)$ are defined by taking the limits $\kappa \rightarrow 1, \infty$ respectively.

If $\Omega \leq 1$ (see Eq. (269)):

1) There exists $\nu \in[0,1)$ such that for any $\bar{\kappa} \in(\nu, 1]\left(\right.$ and $\lim _{g \rightarrow 0^{+}}(\varepsilon \ln \varepsilon) / g=0$ if $\bar{\kappa}=1$ ), the maximum efficiency is

$$
\eta^{-1}(\bar{\kappa})=1+\frac{\beta_{h}}{\beta_{c}-\beta_{h}} \frac{\gamma(1)}{\gamma(\bar{\kappa})}+\Theta(f(g))+\Theta(g)+\Theta(\varepsilon),
$$

where $\gamma(1) \geq \gamma(\bar{\kappa})$ with equality iff $\bar{\kappa}=1$ and $\lim _{g \rightarrow 0+} f(g)=0$. 
2) The corresponding amount of work extracted is

$$
W_{\text {ext }}(\bar{\kappa})=g \frac{n}{\beta_{h}}[\gamma(\bar{\kappa})+\Theta(f(g))] .
$$

If $\Omega>1$ :

1) There exists $\nu^{\prime} \in[0,1)$ such that for any $\bar{\kappa} \in\left(\nu^{\prime}, 1\right]$ (and $\lim _{g \rightarrow 0^{+}}(\varepsilon \ln \varepsilon) / g=0$ if $\left.\bar{\kappa}=1\right)$, the maximum efficiency is

$$
\eta^{-1}(\bar{\kappa})=1+\frac{\beta_{h}}{\beta_{c}-\beta_{h}} \frac{\gamma(1)}{\gamma(\infty)}+\Theta(f(g))+\Theta(g)+\Theta(\varepsilon)
$$

where $\gamma(1)<\gamma(\infty)$.

2) The corresponding amount of work extracted is

$$
W_{\text {ext }}(\bar{\kappa})=g \frac{n}{\beta_{h}}[\gamma(\infty)+\Theta(f(g))]
$$

Proof. Firstly, let us begin by deriving the explicit form for $\gamma(1)$ and $\gamma(\infty)$ :

$$
\gamma(1)=\lim _{\alpha \rightarrow 1} \frac{\alpha}{\alpha-1} B_{\alpha}=\lim _{\alpha \rightarrow 1} B_{\alpha}+\alpha B_{\alpha}^{\prime}=\frac{E^{2}\left(\beta_{c}-\beta_{h}\right)}{\left(1+e^{\beta_{c} E}\right)^{2}} e^{\beta_{c} E}
$$

where we have made use of the L'Hôspital rule. For $\alpha \rightarrow \infty$, since

$$
\lim _{\alpha \rightarrow \infty} B_{\alpha}=\lim _{\alpha \rightarrow \infty} \frac{E}{1+e^{\beta_{c} E}} \frac{e^{\beta_{h} E}-e^{\beta_{c} E} e^{-\alpha\left(\beta_{c}-\beta_{h}\right) E}}{e^{\beta_{h} E}+e^{-\alpha\left(\beta_{c}-\beta_{h}\right) E}}=\frac{E}{1+e^{\beta_{c} E}},
$$

therefore we have

$$
\gamma(\infty)=\lim _{\alpha \rightarrow 1}\left(1+\frac{1}{\alpha-1}\right) \cdot B_{\alpha}=\frac{E}{1+e^{\beta_{c} E}}
$$

By Lemma 14, we know that the infimum of $\gamma(\alpha)$ for $\alpha \in[\bar{\kappa}, \infty)$ and $\bar{\kappa} \in(\nu, 1]$ is either at $\alpha=\bar{\kappa}$ or $\alpha \rightarrow \infty$. Therefore, if we take the ratio of Eqs. (275) and (276) to be

$$
\frac{\gamma(1)}{\gamma(\infty)}=\frac{E\left(\beta_{c}-\beta_{h}\right)}{1+e^{-\beta_{c} E}}=\Omega \leq 1
$$

then $\gamma(\infty) \geq \gamma(1)>\gamma(\bar{\kappa})$, therefore the infimum of $\gamma(\alpha)$ for $\alpha \in[\bar{\kappa}, \infty)$ and $\bar{\kappa} \in(\nu, 1]$ has to be obtained at $\alpha=\bar{\kappa}$. Taking this into account and using the condition which is equivalent to that of near perfect work in Eq. (48), we can use Lemma 14, to calculate the amount of work extracted:

$$
W_{\text {ext }}=\inf _{\alpha \geq 0} W_{\alpha}=g \cdot\left[\inf _{\alpha>\bar{\kappa}} \frac{n}{\beta_{h}} \gamma(\bar{\kappa})+\Theta(f(g))\right]=g \frac{n}{\beta_{h}}[\gamma(\bar{\kappa})+\Theta(f(g))],
$$

where $\lim _{g \rightarrow 0+} f(g)=0$. On the other hand, we can calculate $\Delta C$, which is the change of average energy in the cold bath system, (recall this is done by Taylor expansion around $g=0$ )

$$
\Delta C=n\left(\left\langle E^{2}\right\rangle_{\beta_{c}}-\langle E\rangle_{\beta_{c}}^{2}\right) g+\Theta\left(g^{2}\right)=\frac{n \gamma(1)}{\beta_{c}-\beta_{h}} g+\Theta\left(g^{2}\right) .
$$

Using Eq. (75), we have $\Delta W=(1-\varepsilon) W_{\text {ext }}$. The (inverse) efficiency, according to the definition (61), is thus

$$
\begin{aligned}
\eta^{-1}(\bar{\kappa}) & =1+\frac{\Delta C}{W_{\text {ext }}}-\varepsilon=1+\frac{n \gamma(1) /\left(\beta_{c}-\beta_{h}\right) g+\Theta\left(g^{2}\right)}{n \gamma(\bar{\kappa}) g / \beta_{h}+\Theta(g f(g))}-\varepsilon \\
& =1+\frac{\beta_{h}}{\left(\beta_{c}-\beta_{h}\right)} \frac{\gamma(1)}{\gamma(\bar{\kappa})}+\Theta(f(g))+\Theta(g)+\Theta(\varepsilon),
\end{aligned}
$$


where we have used $\lim _{g \rightarrow 0^{+}} f(g)=0$ which is proven in Lemma 12. We will now investigate the efficiency when $\Omega>1$ is satisfied. Using $\Omega>1$ and Eq. (277), we have that $\gamma(\infty)<\gamma(1)$. Thus from Lemma 14, due to continuity in $\bar{\kappa}$ of $\gamma(\bar{\kappa})$ we conclude that there exists a $\nu^{\prime} \in[0,1)$ such that for any $\bar{\kappa} \in\left(\nu^{\prime}, 1\right]$,

$$
\inf _{\alpha \geq \bar{\kappa}} \gamma(\alpha)=\gamma(\infty)
$$

Therefore, since we are considering near perfect work, Eq. (48) holds and we can use Lemma 12 to calculate the amount of work extracted

$$
W_{\mathrm{ext}}=\inf _{\alpha \geq 0} W_{\alpha}=g \cdot\left[\inf _{\alpha>\bar{\kappa}} \frac{n}{\beta_{h}} \gamma(\bar{\kappa})+f(g)\right]=g \frac{n}{\beta_{h}}\left[\gamma(\infty)+\frac{\beta_{h}}{n} f(g)\right],
$$

where $\lim _{g \rightarrow 0+} f(g)=0$. Thus using the definition of inverse efficiency (Eq. (61)), together with Eq. (279), we have

$$
\begin{aligned}
\eta^{-1}(\bar{\kappa}) & =1+\frac{\Delta C}{W_{\text {ext }}}-\varepsilon=1+\frac{n \gamma(1) /\left(\beta_{c}-\beta_{h}\right) g+\Theta\left(g^{2}\right)}{n \gamma(\infty) g / \beta_{h}+\Theta(g f(g))}-\varepsilon \\
& =1+\frac{\beta_{h}}{\left(\beta_{c}-\beta_{h}\right)} \frac{\gamma(1)}{\gamma(\infty)}+\Theta(f(g))+\Theta(g)+\Theta(\varepsilon),
\end{aligned}
$$

where we have used $\lim _{g \rightarrow 0^{+}} f(g)=0$ which is proven in Lemma 12 .

We will now use Lemma 15 to conclude our main result of this letter.

Lemma 16. Consider the case of near perfect work (Def. (5)) and all cold bath qubits are identical (i.e. $\bar{E}_{i}=E$ for $\left.i=1, \ldots, n\right)$, then:

1) If $\Omega \leq 1$ (see Eq. (269)) the optimal achievable efficiency $\eta_{\max }$ (see Eq. (64)) is the Carnot efficiency:

$$
\eta_{\max }=\left(1+\frac{\beta_{h}}{\beta_{c}-\beta_{h}}\right)^{-1}
$$

What is more, this efficiency is achieved for quasi-static heat engines, i.e. $\eta_{\max }=\eta_{\max }^{\text {stat }}$ (see Eq. (68)).

2) If $\Omega>1$ and the heat engine is quasi-static, then the optimal achievable efficiency is (see Eq. (68))

$$
\eta_{\max }^{\mathrm{stat}}=\left(1+\frac{\beta_{h}}{\beta_{c}-\beta_{h}} \Omega\right)^{-1} .
$$

3) If $\Omega>1$ the maximum achievable efficiency $\eta_{\max }$ (see Eq. (64)), is strictly less that the Carnot efficiency for quasi-static heat engines.

Proof. In Lemma 5, we found that the Carnot Efficiency is an upper bound for the efficiency when we are extracting near perfect work. We also found that Eq. (48) is satisfied iff we are extracting near perfect work. In Lemma 15, we derived the optimal achievable efficiency for quasi-static heat engines as a function of $\bar{\kappa}$ when Eq. (48) is satisfied. By choosing $\bar{\kappa}<1$ arbitrarily close to one, if $\Omega \leq 1$ is satisfied, we will thus achieve an efficiency arbitrarily close to the Carnot efficiency. Thus since the upper bound is equal to the lower bound, we prove part 1) of the Theorem. Part 2) of the Theorem follows from setting $\bar{\kappa}=1$ in Lemma 5 when $\Omega>1$ is satisfied.

By making use of Lemma 15, one can generalize Lemma 16 to consider the more general case stated in A5 (at the beginning of Section E.2.3) where the cold bath still consists of qubits, however the energy gaps of the qubits can be arbitrary. For convenience, we re-write the general cold bath Hamiltonian here: for a set of variables $\bar{E}_{1}>0, \cdots, \bar{E}_{n}>0$,

$$
\hat{H}_{\text {Cold }}=\sum_{k=1}^{n} \mathbb{1}^{\otimes(k-1)} \otimes \hat{H}_{\mathrm{c}}^{k} \otimes \mathbb{1}^{\otimes(n-k)}, \quad \text { where } \quad \hat{H}_{\mathrm{c}}^{k}=\bar{E}_{k}|E\rangle\langle E|,
$$

Under the more general form of the cold bath Eq. (288), we have the following theorem. 
Theorem 2. [Quantum/Nano heat engine efficiency] Consider a quasi-static heat engine (Def. 6) which is extracting near perfect work (Def. (5)), when the cold bath consists of multiple qubits with energy gaps $\left\{\bar{E}_{i}\right\}_{i=1}^{n}$.

1) If $\Omega \leq 1$ (see Eq. (269)) the optimal achievable efficiency $\eta_{\max }^{\text {stat }}$ (see Eq. (68)) is the Carnot efficiency:

$$
\eta_{\max }^{\text {stat }}=\eta_{C}=\left(1+\frac{\beta_{h}}{\beta_{c}-\beta_{h}}\right)^{-1}
$$

2) If $\Omega>1$, then the maximum achievable efficiency is

$$
\eta_{\max }^{\mathrm{stat}}=\left(1+\frac{\beta_{h}}{\beta_{c}-\beta_{h}} \Omega\right)^{-1}
$$

which is strictly less than the Carnot efficiency $\eta_{C}$.

3) Allowing for correlations between the final state of the battery and cold bath cannot improve the efficiencies achieved in 1) and 2) above.

Proof. 1) is relatively simple to prove: as long as there exists a qubit with energy $\bar{E}_{i}$ such that $\frac{\bar{E}_{i}\left(\beta_{c}-\beta_{h}\right)}{1+e^{-\beta_{h} \bar{E}_{i}}} \leq 1$, one way to achieve Carnot efficiency is to simply disregard the rest of the cold bath, and act only on such qubits. The result is a simple application of 1) in Lemma 16. This strategy might not be optimal in terms of work extracted, but it is sufficient for our proof.
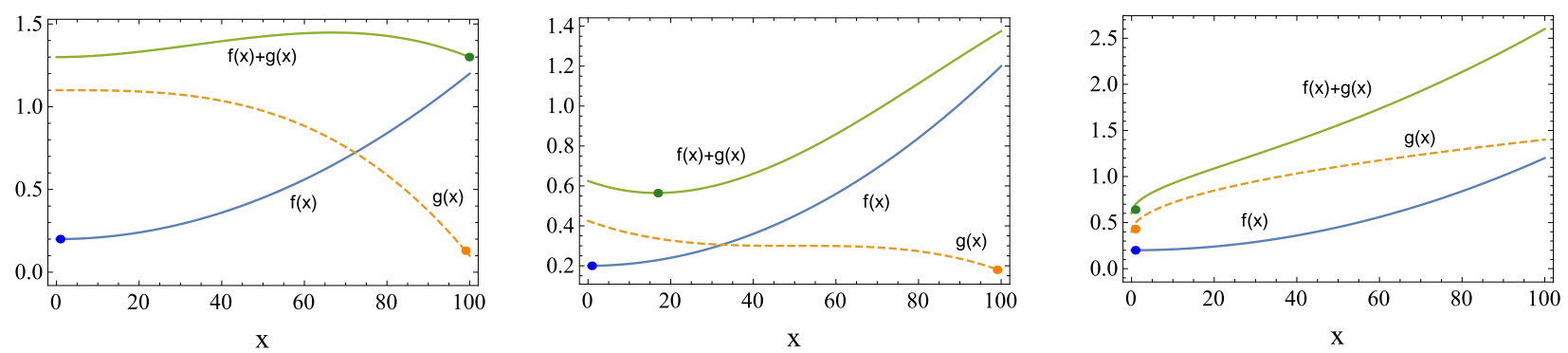

Figure 11: Illustration of the minima of two individual functions $f(x), g(x)$ and minima of $f(x)+g(x)$.

For 2) suppose that $\Omega>1$. From the Eq. (269), we conclude that for all $\bar{E}_{i}$ where $1 \leq i \leq n$, $\Omega_{i}:=\frac{\bar{E}_{i}\left(\beta_{c}-\beta_{h}\right)}{1+e^{-\beta_{h} E_{i}}}>1$. By Lemma 15, we see that this implies that the work extractable for all the individual qubits (which is an optimization problem over all $\alpha \geq 0$ ) is obtained at $\alpha \rightarrow \infty$. In general, considering the qubits collectively does not mean that the collective $W_{\text {ext }}$ is additive. This is because the minima of two functions is not necessarily the minima of these individual functions added together, as illustrated in the l.h.s. and middle diagrams of Figure. 11. However, (as illustrated on r.h.s. diagram of Figure. 11), when all the functions have their minima at the same value, then the collective minima is also obtained at that value.

Next, we show that no matter which subset of qubits $\mathcal{S}$ one picks, Carnot efficiency cannot be achieved. We begin by introducing the notation $\gamma_{i}(\alpha)$, where $\gamma_{i}(\alpha)$ is defined similarly with $\gamma(\alpha)$ in Eq. (270) and (251), and the index $i$ indicates that $E$ is substituted by $\bar{E}_{i}$ in Eq. (251). Furthermore, recall that from Eq. (277), $\Omega_{i}>1$ is equivalent to $\gamma_{i}(1)>\gamma_{i}(\infty)$. Now, consider any subset of qubit indices $\mathcal{S}$, the amount of extractable work (as a function of $g$ ) is

$$
W_{\mathrm{ext}}^{\mathcal{S}}=\frac{g}{\beta_{h}}\left[\sum_{i \in \mathcal{S}} \gamma_{i}(\infty)+f(g)\right],
$$

where $\lim _{g \rightarrow 0+} f(g)=0$. 
On the other hand, we have that $\Delta C$ depends on the individual reduced qubit states, since there are no interaction terms in $\hat{H}_{\text {Cold }}$. Therefore, similar to Eq. (279),

$$
\Delta C^{\mathcal{S}}=\frac{g}{\beta_{c}-\beta_{h}} \sum_{i \in \mathcal{S}} \gamma_{i}(1)+\Theta\left(g^{2}\right) .
$$

Following the same proof in Eq. (284) Lemma 15,

$$
\eta^{-1}(\bar{\kappa})=1+\frac{\Delta C}{W_{\mathrm{ext}}}-\varepsilon=1+\frac{\beta_{h}}{\beta_{c}-\beta_{h}} \frac{\sum_{i \in \mathcal{S}} \gamma_{i}(1)}{\sum_{i \in \mathcal{S}} \gamma_{i}(\infty)}+\Theta(g)+\Theta(\varepsilon) .
$$

As we have observed before, the inverse of the Carnot efficiency $\eta_{C}^{-1}=1+\frac{\beta_{h}}{\beta_{c}-\beta_{h}}$. Furthermore, notice that by Eq. (277), the condition $\Omega_{i}>1$ implies that $\gamma_{i}(1)>\gamma_{i}(\infty)$. Since $\Omega_{i}>1$ is true for all $1 \leq i \leq n$, therefore $\frac{\sum_{i \in \mathcal{S}} \gamma_{i}(1)}{\sum_{i \in \mathcal{S}} \gamma_{i}(\infty)}>1$.

Lastly, part 3) is proven in Section F.

Suppose $n$ is large. Then since we have a spectrum which looks like a quasi-continuum: the full range of the spectrum is very large, compared to the individual energy gaps. If one expects that in such a case, baths are of high temperature (small values of $\beta$ ), then the effects of quantization should give us the classical observations of being able to achieve the Carnot efficiency always. It can be seen, that for $E_{\min }=\min _{i \in\{1, \cdots, n\}} \bar{E}_{i}$, if the quantities $\beta_{c} E_{\min }, \beta_{h} E_{\min } \ll 1$, then

$$
\Omega=\frac{E_{\min }\left(\beta_{c}-\beta_{h}\right)}{1+e^{-\beta_{c} E_{\min }}} \leq E_{\min }\left(\beta_{c}-\beta_{h}\right) \ll 1 .
$$

Whenever $\Omega \leq 1$, we know that Carnot efficiency is achievable.

\section{E.3 Running the heat engine for many cycles quasi-statically}

We have so far proven that a heat engine can achieve the Carnot efficiency when $\Omega \leq 1$. However, as like with macroscopic heat engines, this can only be achieved when the heat engine runs quasistatically. Macroscopic heat engines can then extract a finite amount of work by running the heat engine over many cycles (in fact, over any infinite number of cycles if they want to obtain the Carnot efficiency in order to run quasi-statically). The following lemma, shows that when $\Omega \leq 1$, a nano-scale heat engine with a machine that runs over infinitely many cycles can also achieve the Carnot efficiency, while extracting any finite amount of work $W$ with vanishing entropy increase in the battery.

For simplicity, we will work with the case in which the quasi-continuum battery has a part of its spectrum equal to that of at least $N$ qubits, each with an energy gap $W_{\text {ext. }}$. We work within this subspace. We will run a heat engine between a hot bath, cold bath using a machine which performs $N$ cyclic cycles. Let $\tilde{E}_{j}$ and $\tilde{E}_{k}$ be the smallest and largest energy eigenvalues within this subspace respectively. We let the initial state of the battery be

$$
\rho_{\mathrm{W}}^{0}=\left|\tilde{E}_{j}\right\rangle\left\langle\tilde{E}_{j}\right|
$$

$\hat{H}_{\mathrm{W}}\left|\tilde{E}_{j}\right\rangle=\tilde{E}_{j}\left|\tilde{E}_{j}\right\rangle$ while we wish the final state of the battery to be of the form

$$
\rho_{\mathrm{W}}^{1}=r\left|\tilde{E}_{k}\right\rangle\left\langle\tilde{E}_{k}\right|+(1-r) \rho_{\psi},
$$

where $\hat{H}_{\mathrm{W}}\left|\tilde{E}_{k}\right\rangle=\tilde{E}_{k}\left|\tilde{E}_{k}\right\rangle, \rho_{\psi}$ is some orthogonal state to $\left|\tilde{E}_{k}\right\rangle$ and the value of the probability $r$ is to be specified in the following lemma. We will define the amount of work extracted from the machine for $N$ cycles

$$
W_{\text {cyc }}:=\tilde{E}_{k}-\tilde{E}_{j}
$$

For simplicity, we will consider the case that the cold bath consists of $n$ identical qubits with $\Omega \leq 1$, and during each cycle the machine interacts with one qubit from the cold bath. The running of the heat engine is depicted in Fig. 12 and 13. 


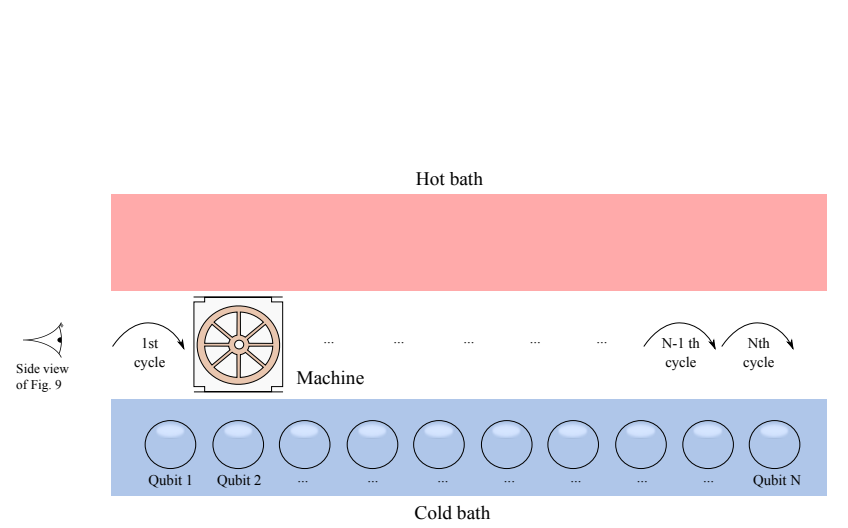

Figure 12: Depiction (top view) of a heat engine comprising of a hot bath, a cold bath consisting of $n$ identical qubits, a machine and a battery. In each cycle, the machine interacts specifically with one qubit from the cold bath, together with the hot bath and battery. After the end of one cycle, the machine is returned to its original state, and acts on a different qubit in the cold bath.

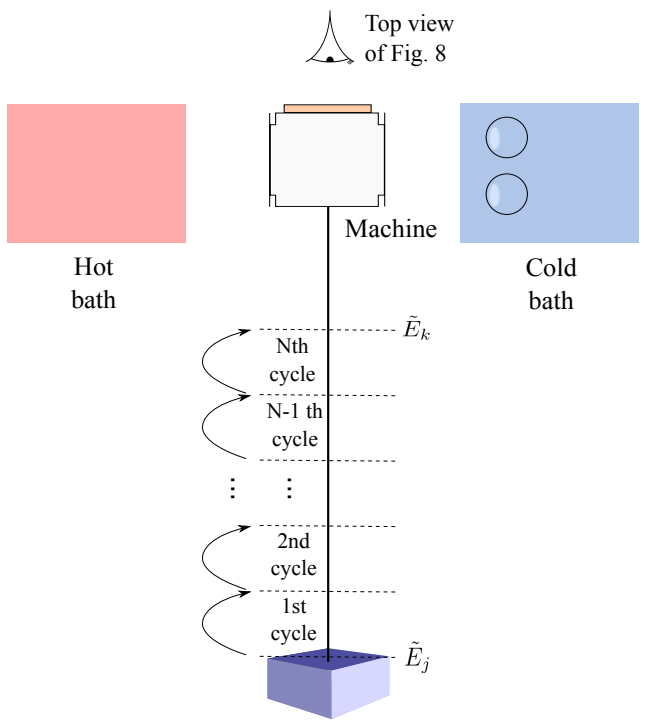

Figure 13: Side view of the heat engine. After each cycle of the machine, the battery, depicted here as a weight moves upward by a small amount. After $N$ machine cycles, it has been lifted from its original position $\left|\tilde{E}_{j}\right\rangle$ to a final state that has most of its weight on $\left|\tilde{E}_{k}\right\rangle$.

Corollary 2. [Many quasi-static heat engine cycles] Let $W$ be the finite amount of work we wish to extract. Then for all $W>0$ and $\delta>0$ there exists an $n$ identical qubit cold bath (with $\Omega \leq 1$ ) and an $N \in \mathbb{N}^{+}$number of machine cycles with $n \geq N$ such that:

1) $\eta_{c} \geq \eta \geq \eta_{c}-\delta$, where the efficiency $\eta$ is the efficiency per cycle and is defined by Eq. (61), and $\eta_{c}=1-\beta_{h} / \beta_{c}$ is the Carnot efficiency,

2) $W_{\text {cyc }} \geq W-\delta$,

3) $S\left(\rho_{\mathrm{W}}^{0}\right)=0, S\left(\rho_{\mathrm{W}}^{1}\right) \leq \delta$, and

4) $r \geq 1-\delta$.

whats more, $\delta \rightarrow 0$ as $N \rightarrow+\infty$.

Proof. Since in the qubit subspace, the spectrum is that of at least $N$ qubits, we can write the initial state in the form

$$
\rho_{\mathrm{W}}^{0}=\left|E_{j}\right\rangle\left\langle\left. E_{j}\right|^{\otimes N},\right.
$$

with $\hat{H}_{\mathrm{W}}\left|E_{j}\right\rangle^{\otimes N}=\tilde{E}_{j}\left|E_{j}\right\rangle^{\otimes N}$. We can now apply the heat engine results of Lemma 15 to the setup. Namely, we can apply the results of one cycle to each of the qubit subspaces of the battery in parallel. From Lemma 15 we conclude that this can be achieved with an efficiency given by Eq. (271) and extract an amount of work per qubit/cycle given by Eq. (272). For simplicity, we will run the heat engine using one qubit of the cold bath at a time. The final state of the battery is thus

$$
\rho_{\mathrm{W}}^{1}=\left[(1-\varepsilon)\left|E_{k}\right\rangle\left\langle E_{k}|+\varepsilon| E_{j}\right\rangle\left\langle E_{j}\right|\right]^{\otimes N} .
$$

Noting that $\left|\tilde{E}_{k}\right\rangle\left\langle\tilde{E}_{k}|=| E_{k}\right\rangle\left\langle\left. E_{k}\right|^{\otimes N}\right.$ by definition, Eq. (299) can be written as

$$
\rho_{\mathrm{W}}^{1}=(1-\varepsilon)^{N}\left|\tilde{E}_{k}\right\rangle\left\langle\tilde{E}_{k}\right|+\left[1-(1-\varepsilon)^{N}\right] \rho_{\psi} .
$$


with $\rho_{\psi}$ orthogonal to $\left|\tilde{E}_{k}\right\rangle$. From Eq. (297) it follows,

$$
W_{\text {cyc }}=N W_{\text {ext }}=\frac{N g}{\beta_{h}}[\gamma(\bar{\kappa})+\Theta(f(g))]
$$

where in the last line we have used Eq. (272). We now set

$$
N=N(g)=\frac{\beta_{h}}{\gamma(\bar{\kappa})} \frac{W}{g}
$$

for all $g>0$ satisfying the constraint $N(g) \in \mathbb{N}^{+}$. For any positive constant $\frac{\beta_{h} W}{\gamma(\bar{\kappa})}>0$, one can always consider the values of $\frac{\beta_{h} W}{\gamma(\bar{\kappa})}>g>0$ so that $N(g)$ is large. This constraint imposes $g=\beta_{h} W /(\gamma(\bar{\kappa}) N)$, where $N$ has to be an integer. Therefore, $g$ now belongs to a subset of the positive real line, rather than the positive real line itself as previously. However, since $g$ monotonically decreases to zero as $N$ increases to infinity, we can still take the limit $g \rightarrow 0^{+}$as before. Thus achieving

$$
W_{\text {cyc }}=W+\Theta(f(g)) \text {. }
$$

Since $\lim _{g \rightarrow 0^{+}} f(g)=0$, we conclude part 2) of Corollary 2. For the entropy of the final state of the battery we have

$$
S\left(\rho_{\mathrm{W}}^{1}\right)=N S\left((1-\varepsilon)\left|E_{k}\right\rangle\left\langle E_{k}|+\varepsilon| E_{j}\right\rangle\left\langle E_{j}\right|\right)=\frac{\beta_{h} W}{\gamma(\bar{\kappa})} \frac{(1-\varepsilon) \ln (1-\varepsilon)+\varepsilon \ln \varepsilon}{g}=\Theta\left(\frac{\varepsilon \ln \varepsilon}{g}\right) .
$$

As stated above the efficiency is given by Eq. (271), and thus we can always choose $\bar{\kappa} \in(0,1)$, and $g$ (recall $\epsilon \rightarrow 0^{+}$as $g \rightarrow 0^{+}$) such that 1) in Corollary 2 is satisfied. Furthermore, recall from the proof of Lemma 12 that

$$
\lim _{g \rightarrow 0^{+}} \frac{\varepsilon \ln \varepsilon}{g}=0,
$$

for all $\bar{\kappa} \in(0,1)$. Thus, from Eq. (304) we conclude that 3) in Corollary 2. We will now prove part 4) of the Corollary. From Eq. (300) and part 4) of the Corollary, we can identify $r=(1-\varepsilon)^{N}$. We thus study the limit

$$
\lim _{g \rightarrow 0^{+}}(1-\varepsilon)^{N}=\left(\lim _{g \rightarrow 0^{+}}(1-\varepsilon)^{1 / g}\right)^{\beta_{h} W / \gamma(\bar{\kappa})}=\left(\lim _{g \rightarrow 0^{+}}(\underbrace{(1-\varepsilon)^{1 / \varepsilon}}_{\rightarrow \mathrm{e}})^{\varepsilon / g}\right)^{\beta_{h} W / \gamma(\bar{\kappa})}=1,
$$

where going to the last line, we have used that fact that Eq. (305) implies that $\varepsilon / g \rightarrow 0$ as $g \rightarrow 0^{+}$. We thus conclude part 4) of the corollary.

Thus by choosing $\delta>0$ sufficiently small in Corollary 2, we can extract any finite amount of work with an arbitrarily small entropy contribution with an efficiency arbitrarily close to the Carnot efficiency as long as $\Omega \leq 1$.

\section{F Extensions to the setup}

Arguably, one may think that the inability to always achieve the Carnot efficiency in the nano regime is due to some subtlety of our setup (even though we have shown that according to the standard free energy one can always achieve the Carnot efficiency with our setup). For such reasons, in the next few sections we show that even under more general conditions than those laid out in Section A, one still cannot achieve the Carnot efficiency when $\Omega>1$.

In Section F.1, we show that allowing for correlations between the final state of the battery and cold bath (and/or the finite dimensional machine) does not allow us to achieve the Carnot efficiency. The main result is Theorem 3 .

In Section F.2, we show that allowing for the battery to be any state with trace distance $\varepsilon$ from $\left|E_{k}\right\rangle\left\langle\left. E_{k}\right|_{\mathrm{W}}\right.$ cannot allow us to achieve the Carnot efficiency when $\Omega>1$. This shows that whenever we are unable to achieve the Carnot efficiency, it is not a artificial defect from an overly specified battery model. The main result is Theorem 4 . 


\section{F.1 Final correlations between battery, cold bath, and machine}

In Section E.2.1, we stated that the final state of the heat engine after tracing out the hot bath was of the form

$$
\operatorname{tr}_{\text {Hot }}\left(\rho_{\text {ColdHotMW }}^{1}\right)=\rho_{\text {Cold }}^{1} \otimes \rho_{\mathrm{M}}^{1} \otimes \rho_{\mathrm{W}}^{1}
$$

where $\rho_{\mathrm{W}}^{1}=\varepsilon\left|E_{j}\right\rangle\left\langle\left. E_{j}\right|_{\mathrm{W}}+(1-\varepsilon) \mid E_{k}\right\rangle\left\langle\left. E_{k}\right|_{\mathrm{W}}\right.$, i.e. the final state of the charged battery was a tensor product with the cold bath. We also demanded that the heat engine is cyclic i.e. that $\rho_{\mathrm{M}}^{1}=\rho_{\mathrm{M}}^{0}$. In this section, we show that if one allows for the final state of the battery, cold bath and machine to become correlated ${ }^{13}$, one still cannot achieve the Carnot efficiency when $\Omega>1$. That is to say, in this section we allow the final state to be

$$
\operatorname{tr}_{\text {Hot }}\left(\rho_{\text {ColdHotMW }}^{1}\right)=\rho_{\text {ColdMW }}^{1}
$$

with only two natural constrains, namely that our heat engine actually extracts work, i.e. that

$$
\rho_{\mathrm{W}}^{1}=\varepsilon\left|E_{j}\right\rangle\left\langle\left. E_{j}\right|_{\mathrm{W}}+(1-\varepsilon) \mid E_{k}\right\rangle\left\langle\left. E_{k}\right|_{\mathrm{W}},\right.
$$

as before, and also that the heat engine is still cyclic, i.e.

$$
\rho_{\mathrm{M}}^{1}=\rho_{\mathrm{M}}^{0} \text {. }
$$

Throughout this section, (unless stated otherwise) we will write $\rho_{\text {ColdMw }}^{1}$ to refer to any generic tripartite quantum state on the cold bath, machine and battery satisfying Eqs. (309) and (310).

- In Section F.1.1, we first define the generalized efficiency where one is allowed to consider correlated final states. We see that although this may potentially affect the amount of extractable work $W_{\text {ext }}$, the amount of heat change in the bath remains the same, by making use of energy conservation and the fact that the global Hamiltonian $H_{\text {ColdHotMw }}$ does not contain interaction terms between subsystems.

- In Section F.1.2, we make use of the generalized second law when $\alpha=1$ (which is also the macroscopic second law), in order to show that final correlations still do not allow the surpassing of Carnot efficiency. This can be proven by noting that the von Neumann entropy is subadditive, and the result is summarized in Lemma 19. A proof sketch can be found in the beginning of Section F.1.2.

- In Section F.1.3, we turn to the case where $\Omega>1$, where without final correlations it is shown in Theorem 2 that Carnot efficiency cannot be achieved.

\section{F.1.1 Defining the generalized efficiency}

Recall that before (see Section C.2), we have shown in Eq. (77) that if the following assumptions hold:

(i) the final reduced state of the battery $\rho_{\mathrm{W}}^{1}$ is fixed by Eq. (42),

(ii) the state of the machine is preserved,

(iii) the final state is of tensor product form, i.e. $\rho_{\text {ColdMW }}^{1}=\rho_{\text {Cold }}^{1} \otimes \rho_{\mathrm{M}}^{1} \otimes \rho_{\mathrm{W}}^{1}$,

then the efficiency for a particular transformation $\rho_{\text {ColdHotMW }}^{0} \rightarrow \rho_{\text {ColdHotMW }}^{1}$ simplifies to being only an explicit function of $\rho_{\text {Cold }}^{1}$ instead of the global final state. This simplified expression of the efficiency in Eq. (77) is then used to evaluate, for example, $\eta^{\operatorname{mac}}\left(\rho_{\text {Cold }}^{1}\right)$ in Eq. (65). Since we now drop Assumption (iii) for the final state being uncorrelated, the efficiency and the work extracted $W_{\text {ext }}$ will now depend on the tripartite final state $\rho_{\text {ColdMw }}^{1}$ instead.

\footnotetext{
${ }^{13}$ Recall that the final state of the cold bath, machine and battery are already allowed to become correlated with the hot bath
} 
Therefore, let us first write a generalized expression for the maximum efficiency corresponding to a transition $\rho_{\text {ColdHotMw }}^{0} \rightarrow \rho_{\text {ColdHotMw }}^{1}$ via the unitary operator $U(t)$ in this generalised setting:

$$
\begin{aligned}
\eta^{\mathrm{qm}}\left(\rho_{\text {ColdMW }}^{1}\right):=\sup _{W_{\text {ext }}} \eta\left(\rho_{\text {Cold }}^{1}, W_{\text {ext }}\right) \text { s.t. } & \operatorname{tr}_{\text {Hot }}\left[U(t) \rho_{\text {ColdHotMW }} U(t)^{\dagger}\right]=\rho_{\text {ColdMW }}^{1}, \\
& {[U(t), \hat{H}]=0, } \\
& \rho_{\mathrm{W}}^{1}=\varepsilon\left|E_{j}\right\rangle\left\langle\left. E_{j}\right|_{\mathrm{W}}+(1-\varepsilon) \mid E_{k}\right\rangle\left\langle\left. E_{k}\right|_{\mathrm{W}},\right. \\
& \rho_{\mathrm{M}}^{1}=\rho_{\mathrm{M}}^{0} .
\end{aligned}
$$

See Fig (1) in main text for a definition of the other quantities appearing in Eq. (311). Recall that the definition of $\eta$ is given by $\eta=W_{\text {ext }} / \Delta H$ as in Eq. (61). In Section C.2 we showed that this can be simplified to

$$
\eta=\left(1-\varepsilon+\Delta C / W_{\text {ext }}\right)^{-1},
$$

where $\Delta C=\Delta C\left(\rho_{\text {Cold }}^{1}\right)$. This equation holds under Assumption (i) and (ii), together with the fact that the global Hamiltonian does not contain interaction terms between both baths, battery, and machine. Since the derivation of Eq. (315) does not require Assumption (iii), it still holds for a general tripartite

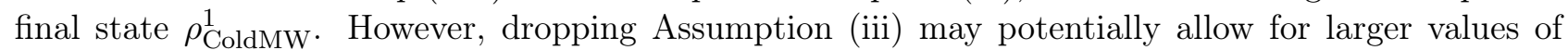
$W_{\text {ext }}$, and therefore subsequently might affect $\eta^{\mathrm{qm}}$. For this reason we write $\eta^{\mathrm{qm}}=\eta^{\mathrm{qm}}\left(\rho_{\text {ColdMW }}^{1}\right)$ to remind ourselves that it is a function of the entire final state $\rho_{\text {ColdMw }}^{1}$.

We have written $\eta=\eta\left(\rho_{\text {Cold }}^{1}, W_{\text {ext }}\right)$ to explicitly show the $W_{\text {ext }}$ dependency of $\eta$. Although not written explicitly in Eq. (311), we should remember that $U(t), \rho_{\mathrm{M}}^{0}, \hat{H}_{\mathrm{Hot}}$ and $\hat{H}_{\mathrm{M}}$ are arbitrary, other than satisfying condition (A.4) in Section A. As such, by maximizing $\eta$ over $W_{\text {ext }}$, these quantities will accommodate their optimal values to maximize $\eta^{\mathrm{qm}}\left(\rho_{\text {ColdMw }}^{1}\right)^{14}$. Throughout this section, we analyze Eq. (311) only in the case of near perfect work (Def. (5)) since the proof that perfect work is not possible (see Lemma 8) also applies to Eq. $(311)^{15}$.

For the purpose of our proofs, we need to define a new family of intermediate efficiencies. They provide the maximum possible efficiency, when considering only a particular instance $\alpha \geq 0$ of the generalized second laws. For any $\alpha \in[0, \infty)$, let us denote

$$
\eta_{\alpha}^{\mathrm{qm}}\left(\rho_{\text {ColdMW }}^{1}\right)=\sup _{W_{\text {ext }}} \eta\left(\rho_{\text {Cold }}^{1}, W_{\text {ext }}\right) \quad \text { s.t. } \quad F_{\alpha}\left(\tau_{\text {Cold }}^{0} \otimes \rho_{\mathrm{M}}^{0} \otimes \rho_{\mathrm{W}}^{0}, \tau_{\text {ColdMW }}^{h}\right) \geq F_{\alpha}\left(\rho_{\text {ColdMW }}^{1}, \tau_{\text {ColdMW }}^{h}\right),
$$

$$
\begin{aligned}
& \operatorname{tr}\left(\hat{H}_{t} \rho_{\text {ColdHotMW }}^{0}\right)=\operatorname{tr}\left(\hat{H}_{t} \rho_{\text {ColdHotMW }}^{1}\right), \\
& \rho_{\mathrm{W}}^{1}=\varepsilon\left|E_{j}\right\rangle\left\langle\left. E_{j}\right|_{\mathrm{W}}+(1-\varepsilon) \mid E_{k}\right\rangle\left\langle\left. E_{k}\right|_{\mathrm{W}},\right. \\
& \rho_{\mathrm{M}}^{1}=\rho_{\mathrm{M}}^{0} .
\end{aligned}
$$

See Eq. (56) for definition of $F_{\alpha}$. We denote $\eta_{\infty}^{\mathrm{qm}}=\lim _{\alpha \rightarrow \infty} \eta_{\alpha}^{\mathrm{qm}}$. The condition Eq. (317), is always satisfied when all the second laws are satisfied. We add the condition as a constraint here, since we will need it in order to write the efficiency $\eta$ in the form of Eq. (315).

\section{F.1.2 Final correlations do not allow the surpassing of Carnot efficiency}

Here, we show that Carnot efficiency cannot be surpassed in a quasi-static heat engine even when we

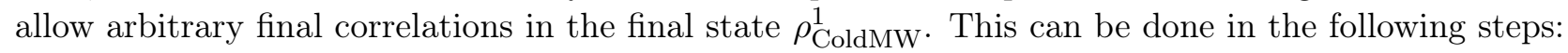

1. Using the definitions of generalized efficiency (allowing correlations) in Eq. (311) and generalized intermediate efficiencies in Eq. (316), we prove an inequality between $\eta^{\mathrm{qm}}\left(\rho_{\text {ColdMw }}^{1}\right)$ and $\eta_{\alpha}^{\mathrm{qm}}\left(\rho_{\text {ColdMW }}^{1}\right)$, for all $\alpha \geq 0$. This is done in Lemma 17. From this, we also conclude that $\eta^{\mathrm{qm}}\left(\rho_{\text {ColdMW }}^{1}\right) \leq \eta_{1}^{\mathrm{qm}}\left(\rho_{\text {ColdMW }}^{1}\right)$.

\footnotetext{
${ }^{14}$ This is an advantage, since it rules out cases such as when the Hamiltonian does not support a thermal state (e.g. when the corresponding thermal state's partition function diverges). In this section we consider any cold bath Hamiltonian $\hat{H}_{\text {Cold }}$ that satisfies (A.6) in Section A (i.e. finite dimensional). As such it will always have a well defined thermal state.

${ }^{15}$ For the sake of full generality, some of the lemmas in this section are proven irrespective to whether we are considering perfect or near perfect work
} 
2. On the other hand, we show that for any final state of the cold bath, machine and battery $\rho_{\text {Coldmw, }}^{1}$, the generalized intermediate efficiency for $\alpha=1$ only increases, if we consider the tensor product of

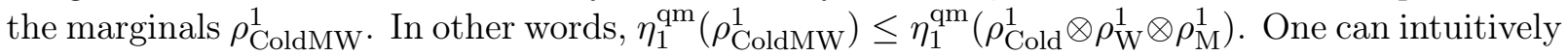
see why this is true: it comes from the fact that the von Neumann entropy is subadditive, therefore the final state $\rho_{\text {Cold }}^{1} \otimes \rho_{\mathrm{W}}^{1} \otimes \rho_{\mathrm{M}}^{1}$ contains more entropy than $\rho_{\text {ColdMW }}^{1}$. Therefore according to the $\alpha=1$ second law, one can potentially draw more work by going to the state $\rho_{\text {Cold }}^{1} \otimes \rho_{\mathrm{W}}^{1} \otimes \rho_{\mathrm{M}}^{1}$ instead of a correlated state $\rho_{\text {ColdMW }}^{1}$.

3. Since the argument for $\eta_{1}^{\mathrm{qm}}\left(\rho_{\text {Cold }}^{1} \otimes \rho_{\mathrm{W}}^{1} \otimes \rho_{\mathrm{M}}^{1}\right)$ is of tensor product form, Assumption (iii) holds as before, and therefore the efficiency only depends on the final state of the cold bath $\rho_{\text {Cold }}^{1}$. This means that Eq. (316) for $\alpha=1$ reduces to Eq. (65). Lastly, by using Lemma 22, this allows us to further show in Lemma 19 that even by allowing correlations in $\rho_{\text {ColdMw }}^{1}$, the efficiency $\eta^{\mathrm{qm}}\left(\rho_{\text {ColdMW }}^{1}\right)$ can never surpass the Carnot value.

Firstly, let us fix the following notation: for an $R$-partite state $\rho_{\mathrm{A}_{1} \mathrm{~A}_{2} \ldots \mathrm{A}_{R}}$, define the uncorrelated counterpart

$$
\underline{\rho_{\mathrm{A}_{1} \mathrm{~A}_{2} \ldots \mathrm{A}_{R}}}:=\bigotimes_{i=1}^{R} \rho_{\mathrm{A}_{i}} .
$$

Comparing $\rho_{\mathrm{A}_{1} \mathrm{~A}_{2} \ldots \mathrm{A}_{R}}$ and $\rho_{\mathrm{A}_{1} \mathrm{~A}_{2} \ldots \mathrm{A}_{R}}$, one will see that each subsystem has the same reduced state, but the global state is different. Another useful thing is to note that if one is given a Hamiltonian which does not contain any interaction terms between each subsystem, i.e.

$$
\hat{H}_{\mathrm{A}_{1} \mathrm{~A}_{2} \ldots \mathrm{A}_{R}}=\sum_{i=1}^{R} \mathbb{1}_{\mathrm{A}_{1}} \otimes \cdots \hat{H}_{\mathrm{A}_{i}} \cdots \mathbb{1}_{\mathrm{A}_{R}}
$$

then we may conclude that

$$
\operatorname{tr}\left(\hat{H}_{\mathrm{A}_{1} \mathrm{~A}_{2} \ldots \mathrm{A}_{R}} \rho_{\mathrm{A}_{1} \mathrm{~A}_{2} \ldots \mathrm{A}_{R}}\right)=\sum_{i=1}^{R} \operatorname{tr}\left(\hat{H}_{\mathrm{A}_{i}} \rho_{\mathrm{A}_{i}}\right)=\sum_{i=1}^{R} \operatorname{tr}\left(\hat{H}_{\mathrm{A}_{i}} \underline{\rho_{\mathrm{A}_{i}}}\right)=\operatorname{tr}\left(\hat{H}_{\mathrm{A}_{1} \mathrm{~A}_{2} \ldots \mathrm{A}_{R}} \underline{\rho_{\mathrm{A}_{1} \mathrm{~A}_{2} \ldots \mathrm{A}_{R}}}\right) .
$$

Lemma 17. For all $\alpha \geq 0$ and all states $\rho_{\text {ColdHotMW }}^{1}$,

$$
\eta^{\mathrm{qm}}\left(\rho_{\text {ColdMW }}^{1}\right) \leq \eta_{\alpha}^{\mathrm{qm}}\left(\rho_{\text {ColdMW }}^{1}\right)
$$

where $\eta^{\mathrm{qm}}$ and $\eta_{\alpha}^{\mathrm{qm}}$ are defined in Eqs. (311) and (316) respectively.

Proof. For every $\alpha \geq 0$, Eq. $F_{\alpha}\left(\tau_{\text {Cold }}^{0} \otimes \rho_{\mathrm{M}}^{0} \otimes \rho_{\mathrm{W}}^{0}, \tau_{\text {ColdMW }}^{h}\right) \geq F_{\alpha}\left(\rho_{\text {ColdMW }}^{1}, \tau_{\text {ColdMW }}^{h}\right)$ in Eq. (316) is a necessary condition for the transformation $\rho_{\text {ColdMW }}^{0} \rightarrow \rho_{\text {ColdMW }}^{1}$ to occur under an energy preserving unitary with the aid of a catalyst [7]. Energy preserving unitaries also preserve the average energy and thus the Eq. $\operatorname{tr}\left(\hat{H}_{t} \rho_{\text {ColdHotMW }}^{0}\right)=\operatorname{tr}\left(\hat{H}_{t} \rho_{\text {ColdHotMW }}^{1}\right)$ in Eq. (316) is also a necessary condition. If a unitary $U(t)$ satisfies the conditions in Eq. (311), then by the second laws it satisfies Eq. (316) for any particular $\alpha \geq 0$. As a consequence of these observations, the set of allowed unitaries $U(t)$ in Eq. (311) is a subset of allowed unitaries facilitating the catalytic thermal operation which transforms $\rho_{\text {ColdMw }}^{0}$ to $\rho_{\text {ColdMw }}^{1}$ in Eq. (316).

Lemma 18. For any final state $\rho_{\text {ColdMw }}^{1}$, consider the quantity $\eta_{1}^{\mathrm{qm}}\left(\rho_{\text {ColdMW }}^{1}\right)$ defined in Eq. (316). Consider the optimization problem

$$
\begin{aligned}
a\left(\rho_{\text {ColdMW }}^{1}\right):=\sup _{W_{\text {ext }}} \eta\left(\rho_{\text {Cold }}^{1}, W_{\text {ext }}\right) \text { s.t. } & F_{1}\left(\tau_{\text {Cold }}^{0} \otimes \rho_{\mathrm{M}}^{0} \otimes \rho_{\mathrm{W}}^{0}, \tau_{\text {ColdMW }}^{h}\right)=F_{1}\left(\rho_{\text {ColdMW }}^{1}, \tau_{\text {ColdMW }}^{h}\right), \\
& \operatorname{tr}\left(\hat{H}_{t} \rho_{\text {ColdHotMW }}^{0}\right)=\operatorname{tr}\left(\hat{H}_{t} \rho_{\text {ColdHotMW }}^{1}\right) \\
& \rho_{\mathrm{W}}^{1}=\varepsilon\left|E_{j}\right\rangle\left\langle\left. E_{j}\right|_{\mathrm{W}}+(1-\varepsilon) \mid E_{k}\right\rangle\left\langle\left. E_{k}\right|_{\mathrm{W}},\right. \\
& \rho_{\mathrm{M}}^{1}=\rho_{\mathrm{M}}^{0} .
\end{aligned}
$$

Then, $\eta_{1}^{\mathrm{qm}}\left(\rho_{\text {ColdMW }}^{1}\right)=a\left(\rho_{\text {ColdMw }}^{1}\right)$. 
Proof. We begin by noting that the free energy $F_{1}$ can be written as

$$
F_{1}\left(\rho, \tau^{h}\right)=\operatorname{tr}(\hat{H} \rho)-\frac{1}{\beta_{h}} S(\rho)
$$

where $\langle\hat{H}\rangle_{\rho}:=\operatorname{tr}[\hat{H} \rho]$, and $S(\rho)=-\operatorname{tr}(\rho \ln \rho)$ is the von Neumann entropy, while $\tau^{h}$ is the thermal state at inverse temperature $\beta_{h}$ for the Hamiltonian $\hat{H}$. Also, let us recall that $W_{\text {ext }}=E_{k}^{\mathrm{W}}-E_{j}^{\mathrm{W}}>0$ where $E_{j}^{\mathrm{W}}$ is a constant.

Next, we consider the free energies $F_{1}\left(\tau_{\text {Cold }}^{0} \otimes \rho_{\mathrm{M}}^{0} \otimes \rho_{\mathrm{W}}^{0}, \tau_{\text {ColdMW }}^{h}\right)$ and $F_{1}\left(\rho_{\text {ColdMW }}^{1}, \tau_{\text {ColdMW }}^{h}\right)$ respectively, and how they relate to $W_{\text {ext }}$. First of all, note that the quantity $F_{1}\left(\tau_{\text {Cold }}^{0} \otimes \rho_{\mathrm{M}}^{0} \otimes \rho_{\mathrm{W}}^{0}, \tau_{\text {ColdMW }}^{h}\right)$ is simply a constant that does not depend on $W_{\text {ext }}$. This is because

$$
\begin{aligned}
F_{1}\left(\tau_{\text {Cold }}^{0} \otimes \rho_{\mathrm{M}}^{0} \otimes \rho_{\mathrm{W}}^{0}, \tau_{\text {ColdMW }}^{h}\right) & =F_{1}\left(\tau_{\text {Cold }}^{0}, \tau_{\text {Cold }}^{h}\right)+F_{1}\left(\tau_{\mathrm{M}}^{0}, \tau_{\mathrm{M}}^{h}\right)+F_{1}\left(\tau_{\mathrm{W}}^{0}, \tau_{\mathrm{W}}^{h}\right) \\
& =F_{1}\left(\tau_{\text {Cold }}^{0}, \tau_{\text {Cold }}^{h}\right)+F_{1}\left(\tau_{\mathrm{M}}^{0}, \tau_{\mathrm{M}}^{h}\right)+\operatorname{tr}\left(\hat{H}_{\mathrm{W}} \rho_{\mathrm{W}}^{0}\right)-\beta_{h}^{-1} S\left(\rho_{\mathrm{W}}^{0}\right) \\
& =F_{1}\left(\tau_{\text {Cold }}^{0}, \tau_{\text {Cold }}^{h}\right)+F_{1}\left(\tau_{\mathrm{M}}^{0}, \tau_{\mathrm{M}}^{h}\right)+E_{j}^{\mathrm{W}},
\end{aligned}
$$

where the first two terms do not depend on the battery Hamiltonian at all, while in the last equality we have made use of the fact that $\rho_{\mathrm{W}}^{0}=\left|E_{j}\right\rangle\left\langle\left. E_{j}\right|_{\mathrm{W}}\right.$. On the other hand,

$$
\begin{aligned}
F_{1}\left(\rho_{\text {ColdMw }}^{1}, \tau_{\text {ColdMw }}^{h}\right) & =\operatorname{tr}\left(\hat{H}_{\text {ColdMw }} \rho_{\text {ColdMw }}^{1}\right)-\beta_{h}^{-1} S\left(\rho_{\text {ColdMw }}^{1}\right) \\
& =\operatorname{tr}\left(\hat{H}_{\text {Cold }} \rho_{\text {Cold }}^{1}\right)+\operatorname{tr}\left(\hat{H}_{\mathrm{M}} \rho_{\mathrm{M}}^{1}\right)+\operatorname{tr}\left(\hat{H}_{\mathrm{W}} \rho_{\mathrm{W}}^{1}\right)-\beta_{h}^{-1} S\left(\rho_{\text {ColdMW }}^{1}\right) \\
& =\operatorname{tr}\left(\hat{H}_{\text {Cold }} \rho_{\text {Cold }}^{1}\right)+\operatorname{tr}\left(\hat{H}_{\mathrm{M}} \rho_{\mathrm{M}}^{1}\right)-\beta_{h}^{-1} S\left(\rho_{\text {ColdMW }}^{1}\right)+\varepsilon E_{j}^{\mathrm{W}}+(1-\varepsilon) E_{k}^{\mathrm{W}} .
\end{aligned}
$$

Note that again, $\operatorname{tr}\left(\hat{H}_{\text {Cold }} \rho_{\text {Cold }}^{1}\right)$ and $\operatorname{tr}\left(\hat{H}_{\mathrm{M}} \rho_{\mathrm{M}}^{1}\right)$ do not depend on the battery Hamiltonian and therefore do not depend on $E_{k}^{\mathrm{W}}$. Similarly, $S\left(\rho_{\text {ColdMw }}^{1}\right)$ depends only on the eigenvalues of the state, and is independent of $E_{k}^{\mathrm{W}}$. Since $\varepsilon \in[0,1)$, we may conclude the following: $F\left(\rho_{\text {ColdMW }}^{1}, \tau_{\text {ColdMW }}^{h}\right)$ is a continuous function that strictly increases w.r.t. $E_{k}^{\mathrm{W}}$, and therefore it also strictly increases w.r.t. $W_{\text {ext }}$.

To prove this lemma, it suffices to show that the supremum over $W_{\text {ext }}$ in Eq. (316) for $\alpha=1$ has to be achieved when $F_{1}\left(\tau_{\text {Cold }}^{0} \otimes \rho_{\mathrm{M}}^{0} \otimes \rho_{\mathrm{W}}^{0}, \tau_{\text {ColdMW }}^{h}\right)=F_{1}\left(\rho_{\text {ColdMW }}^{1}, \tau_{\text {ColdMW }}^{h}\right)$. We prove this by contradiction. Suppose that $\hat{W}_{\text {ext }}$ achieves the supremum for $\eta_{1}^{\mathrm{qm}}$, and for this value of $\hat{W}_{\text {ext }}$, $F_{1}\left(\tau_{\text {Cold }}^{0} \otimes \rho_{\mathrm{M}}^{0} \otimes \rho_{\mathrm{W}}^{0}, \tau_{\text {ColdMw }}^{h}\right)>F_{1}\left(\rho_{\text {ColdMW }}^{1}, \tau_{\text {ColdMw }}^{h}\right)$. Since we know that $F\left(\rho_{\text {ColdMW }}^{1}, \tau_{\text {ColdMW }}^{h}\right)$ strictly increases w.r.t. $W_{\text {ext }}$, there must exist an $W_{\text {ext }}^{\prime}>\hat{W}_{\text {ext }}$ such that $F_{1}\left(\tau_{\text {Cold }}^{0} \otimes \rho_{\mathrm{M}}^{0} \otimes \rho_{\mathrm{W}}^{0}, \tau_{\text {ColdMW }}^{h}\right) \geq$ $F_{1}\left(\rho_{\text {ColdMw }}^{1}, \tau_{\text {ColdMw }}^{h}\right)$. Furthermore, since by Eq. (315) we know that the efficiency is monotonically increasing w.r.t. $W_{\text {ext }}$ as well, it follows that $W_{\text {ext }}^{\prime}$ achieves a higher value of efficiency compared to $\hat{W}_{\text {ext }}$ while satisfying the required constraints at the same time. This is a contradiction, and therefore we conclude that the optimization for $\eta_{1}^{\mathrm{qm}}$ can be simplified to $a\left(\rho_{\text {ColdMW }}^{1}\right)$, where the constraint on $F_{1}$ holds with equality.

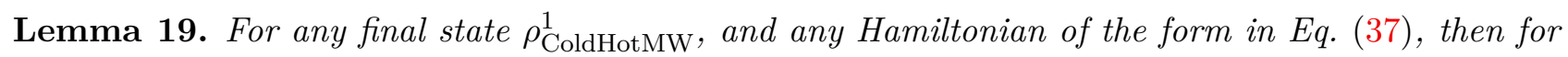
perfect or near perfect work extraction (see Defs. 4 and 5), we have

$$
\eta^{\mathrm{qm}}\left(\rho_{\text {ColdMW }}^{1}\right) \stackrel{(1)}{\leq} \eta_{1}^{\mathrm{qm}}\left(\rho_{\text {ColdMw }}^{1}\right) \stackrel{(2)}{\leq} \eta_{1}^{\mathrm{qm}}\left(\underline{\rho_{\text {ColdMW }}^{1}}\right) \stackrel{(3)}{=} \eta^{\mathrm{mac}}\left(\underline{\rho_{\text {Cold }}^{1}}\right) \stackrel{(4)}{\leq} 1-\frac{\beta_{h}}{\beta_{c}},
$$

with equality in (2) iff $\rho_{\text {ColdMW }}^{1}=\rho_{\text {ColdMw. The quantities }}^{1} \eta_{1}^{\mathrm{qm}}$ and $\eta^{\mathrm{mac}}$ are defined in Eq. (316) and Eq. (65) respectively.

Proof. Note that inequality (1) is a direct consequence of Lemma 17, while inequality (4) holds because of Lemma 6. It remains to prove inequalities (2) and (3).

Proof of inequality (2): Using the definition in Eq. (316) together with Lemma 18, let us compare the 
quantities

$$
\begin{aligned}
\eta_{1}^{\mathrm{qm}}\left(\rho_{\text {ColdMW }}^{1}\right)=\sup _{W_{\mathrm{ext}}} \eta\left(\rho_{\text {Cold }}^{1}, W_{\text {ext }}\right) \quad \text { s.t. } & F_{1}\left(\tau_{\text {Cold }}^{0} \otimes \rho_{\mathrm{M}}^{0} \otimes \rho_{\mathrm{W}}^{0}, \tau_{\text {ColdMW }}^{h}\right)=F_{1}\left(\rho_{\text {ColdMW }}^{1}, \tau_{\text {ColdMW }}^{h}\right), \\
& \operatorname{tr}\left(\hat{H}_{t} \rho_{\text {ColdHotMW }}^{0}\right)=\operatorname{tr}\left(\hat{H}_{t} \rho_{\text {ColdHotMW }}^{1}\right) \\
& \rho_{\mathrm{W}}^{1}=\varepsilon\left|E_{j}\right\rangle\left\langle\left. E_{j}\right|_{\mathrm{W}}+(1-\varepsilon) \mid E_{k}\right\rangle\left\langle\left. E_{k}\right|_{\mathrm{W}}\right. \\
& \rho_{\mathrm{M}}^{1}=\rho_{\mathrm{M}}^{0}
\end{aligned}
$$

and

$$
\begin{aligned}
\eta_{1}^{\mathrm{qm}}\left(\underline{\rho_{\text {ColdMW }}^{1}}\right)=\sup _{W_{\mathrm{ext}}} \eta\left(\underline{\rho_{\text {Cold }}^{1}}, W_{\text {ext }}\right) \text { s.t. } & F_{1}\left(\tau_{\text {Cold }}^{0} \otimes \rho_{\mathrm{M}}^{0} \otimes \rho_{\mathrm{W}}^{0}, \tau_{\text {ColdMW }}^{h}\right)=F_{1}\left(\underline{\rho_{\text {ColdMW }}^{1}}, \tau_{\text {ColdMW }}^{h}\right), \\
& \operatorname{tr}\left(\hat{H}_{t} \rho_{\text {ColdHotMW }}^{0}\right)=\operatorname{tr}\left(\hat{H}_{t} \rho_{\text {ColdHotMW }}^{1}\right) \\
& \rho_{\mathrm{W}}^{1}=\varepsilon\left|E_{j}\right\rangle\left\langle\left. E_{j}\right|_{\mathrm{W}}+(1-\varepsilon) \mid E_{k}\right\rangle\left\langle\left. E_{k}\right|_{\mathrm{w}},\right. \\
& \rho_{\mathrm{M}}^{1}=\rho_{\mathrm{M}}^{0}
\end{aligned}
$$

We first make the following observations:

- By our definition of $\rho_{\text {ColdMw, }}^{1}$, we have that $\rho_{\text {Cold }}^{1}=\rho_{\text {Cold }}^{1}$. Therefore, the term $\Delta C$ in Eq. (315) which is only a function of the reduced state on the cold bath is the same for both efficiencies in Eq. (336) and Eq. (340). Therefore, to compare the efficiencies, we need only to compare the value of $W_{\text {ext }}$ that satisfies the free energy constraint in both optimization problems.

- In [65] (pg. 395) it has been proven that the von Neumann entropy is subadditive

$$
S\left(\rho_{A B}\right) \leq S\left(\underline{\rho_{A B}}\right),
$$

with equality iff $\rho_{A B}=\rho_{A B}$. Furthermore, since $\hat{H}_{\text {ColdMw }}$ does not contain interaction terms, as we have demonstrated earlier in Eq. (322),

$$
\operatorname{tr}\left(\hat{H}_{\text {ColdMw }} \rho_{\text {ColdMw }}^{1}\right)=\operatorname{tr}\left(\hat{H}_{\text {ColdMw }} \underline{\rho_{\text {ColdMW }}^{1}}\right) .
$$

Thus, by Eq. (328) we conclude that

$$
F_{1}\left(\underline{\rho_{\text {ColdMW }}^{1}}\right) \leq F_{1}\left(\rho_{\text {ColdMW }}^{1}\right),
$$

with equality iff $\rho_{\text {ColdMW }}^{1}=\rho_{\text {ColdMW. }}^{1}$.

- For any final state $\rho_{\text {ColdMw }}^{1}$ where $\rho_{\mathrm{W}}^{1}=\varepsilon\left|E_{j}\right\rangle\left\langle\left. E_{j}\right|_{\mathrm{W}}+(1-\varepsilon) \mid E_{k}\right\rangle\left\langle\left. E_{k}\right|_{\mathrm{W}}\right.$, we have seen in the proof of Lemma 18 that $F_{1}\left(\rho_{\text {ColdMW }}^{1}, \tau_{\text {ColdMW }}^{h}\right)$ is a continuous function that strictly increases with $W_{\text {ext }}$.

With these three observations we can now prove inequality (2). Note that when $\rho_{\text {ColdMw }}^{1}=\rho_{\text {ColdMW }}^{1}$, equality holds trivially. Therefore, let us consider the case where $\rho_{\text {ColdMw }}^{1} \neq \rho_{\text {ColdMw }}^{1}$. Suppose $\hat{W}_{\text {ext }}$ achieves the supremum in $\eta_{1}^{\mathrm{qm}}\left(\rho_{\text {ColdMW }}^{1}\right)$, and for such a value of $\hat{W}_{\text {ext }}$,

$$
F_{1}\left(\tau_{\text {Cold }}^{0} \otimes \rho_{\mathrm{M}}^{0} \otimes \rho_{\mathrm{W}}^{0}, \tau_{\text {ColdMW }}^{h}\right)=F_{1}\left(\rho_{\text {ColdMW }}^{1}, \tau_{\text {ColdMW }}^{h}\right)>F_{1}\left(\underline{\rho_{\text {ColdMW }}^{1}}, \tau_{\text {ColdMW }}^{h}\right) .
$$

We note also that since $F_{1}\left(\rho_{\text {ColdMW }}^{1}, \tau_{\text {ColdMW }}^{h}\right)$ strictly increases with $W_{\text {ext }}$, and therefore there exists some $W_{\text {ext }}^{\prime}>\hat{W}_{\text {ext }}$ such that $F_{1}\left(\tau_{\text {Cold }}^{0} \otimes \rho_{\mathrm{M}}^{0} \otimes \rho_{\mathrm{W}}^{0}, \tau_{\text {ColdMW }}^{h}\right)=F_{1}\left(\rho_{\text {ColdMW }}^{1}, \tau_{\text {ColdMW }}^{h}\right)$. Therefore, $W_{\text {ext }}^{\prime}$ is a feasible solution for Eq. (340), i.e. it satisfies the constraints in the optimization problem. In conclusion, we have 


$$
\eta_{1}^{\mathrm{qm}}\left(\rho_{\text {ColdMW }}^{1}\right)=\left[1-\varepsilon+\frac{\Delta C}{\hat{W}_{\text {ext }}}\right]^{-1} \leq\left[1-\varepsilon+\frac{\Delta C}{W_{\text {ext }}^{\prime}}\right]^{-1} \leq \eta_{1}^{\mathrm{qm}}\left(\underline{\rho_{\text {ColdMW }}^{1}}\right) .
$$

Proof of equality (3): Consider the quantity $\eta_{1}^{\mathrm{qm}}\left(\rho_{\text {ColdMw }}^{1}\right)$. Since the state $\rho_{\text {ColdMw }}^{1}$ takes on a product structure form between all the subsystems now, Assumption (iii) in the beginning of Section F.1.1 holds again. By Eqns. (338) and (339), we know that Assumptions (i) and (ii) also hold. Therefore, we know that under these assumptions the efficiency does not depend anymore on the global state $\underline{\rho_{\text {ColdMW }}^{1}}$, but only $\rho_{\text {Cold }}^{1}$. Again comparing the conditions of $\eta^{\mathrm{mac}}\left(\underline{\rho_{\text {Cold }}^{1}}\right)$ and $\eta_{1}^{\mathrm{qm}}\left(\rho_{\text {ColdMW }}^{1}\right)$, we see that they are exactly the same quantity.

Therefore, Lemma 19 tells us that correlations between the final states of the cold bath, machine and battery cannot allow you to achieve an efficiency greater than the Carnot efficiency.

\section{F.1.3 Achievability of Carnot efficiency still depends on more than temperature}

Earlier in Section F.1.2, we proved in Lemma 19 that Carnot efficiency gives an upper bound to the efficiency of any arbitrary final state $\rho_{\text {ColdMw }}^{1}$. In this section, we want to prove that when $\Omega>1$ holds, quasi-static heat engines cannot achieve the Carnot efficiency even when allowing correlations between the final states of the battery and the cold bath. This can be done in the following steps:

- According to Lemma 19, Carnot efficiency can be attained only when all the inequalities in Eq. (335) are satisfied with equalities. We use this to prove in Lemma 20 that in order to achieve the Carnot efficiency, we may only consider the limit where correlations in the final state vanish. Not only so, the magnitude of these correlations also have to vanish quickly enough in order for Carnot efficiency to be achieved. In particular, we define a parameter $k$ which quantifies the amount of correlations, and show that $k$ has to vanish faster than the quasi-static parameter $g$, in order to achieve the Carnot efficiency $\eta_{C}$.

- Next, in Lemma 21, we show that if the parameter $k$ vanishes faster than the quasi-static parameter $g$, then whenever $\Omega>1$, one can derive an upper bound for the intermediate efficiency $\eta_{\infty}^{\mathrm{qm}}\left(\rho_{\text {ColdMW }}^{1}\right)$ which considers the amount of work extractable by invoking only the generalized second law of $\alpha \rightarrow \infty$. Combining Lemma 20 and Lemma 21, we conclude in Corollary 3 that when $\Omega>1, \eta^{\mathrm{qm}} \leq \eta_{\infty}^{\mathrm{qm}} \leq \eta_{C}$ is strictly upper bounded away from the Carnot efficiency.

Before we begin, let us note that by definition, the initial state $\rho_{\mathrm{ColdW}}^{0}$ is diagonal in its energy eigenbasis. Furthermore, the state $\rho_{\text {ColdMw }}^{0}$ is of the form $\rho_{\text {Cold }}^{0} \otimes \rho_{\mathrm{M}}^{0} \otimes \rho_{\mathrm{W}}^{0}$. Since w.l.o.g. we can assume that $\hat{H}_{\mathrm{M}}$ is proportional to the identity (or called the trivial Hamiltonian, see [7]), $\rho_{\mathrm{M}}^{0}$ can always be written as a diagonal state in an energy eigenbasis of its Hamiltonian. Therefore the state $\rho_{\text {ColdMw }}^{0}$ is always diagonal in the energy eigenbasis of the Hamiltonian $\hat{H}_{\text {ColdMw }}:=\hat{H}_{\text {Cold }}+\hat{H}_{\mathrm{M}}+\hat{H}_{\mathrm{W}}$. Since catalytic thermal operations cannot create coherences [7], $\rho_{\text {Coldmw }}^{1}$ has to be also diagonal in the energy eigenbasis of $\hat{H}_{\text {ColdMw. }}$.

We observe that any $\rho_{\text {Coldmw }}^{1}$ can always be written as

$$
\rho_{\text {ColdMW }}^{1}=\left(1-k^{*}\right) \underline{\rho_{\text {ColdMW }}^{1}}+k^{*} \rho_{\text {ColdMW }}^{\text {corr }}
$$

where $k^{*}=\min \left\{k \in[0,1] \mid \rho_{\text {ColdMw }}^{1}=(1-k) \underline{\rho_{\text {ColdMw }}^{1}}+k Q, Q \geq \mathbf{0}\right\}$. This means that $\rho_{\text {ColdMw }}^{1}$ can be written as a convex combination of two states: one being $\rho_{\text {ColdMW }}^{1}$, and the other $\rho_{\text {Coldmw }}^{\text {corr }}$ containing all other correlations. Note that such a $k^{*}$ always exists, in particular, $k=1$ is always a feasible solution.

We now define a particular parametrization of the final states,

$$
\rho_{\text {ColdMW }}^{1}\left(k, \rho_{\text {ColdMW }}^{\text {no corr }}, \rho_{\text {ColdMW }}^{\text {corr }}\right):=(1-k) \rho_{\text {ColdMW }}^{\text {no corr }}+k \rho_{\text {ColdMW }}^{\text {corr }}, \quad k \in\left[0, k^{*}\right]
$$


where the following holds:

$$
\begin{aligned}
& \text { (i) } \rho_{\text {ColdMW }}^{\text {no corr }}=\rho_{\text {ColdMW }}^{1}, \\
& \text { (ii) } \rho_{\text {ColdMW }}^{\text {corr }} \neq \rho_{\text {ColdMW }}^{\text {no corr }}, \\
& \text { (iii) } \rho_{\mathrm{M}}^{1}=(1-k) \rho_{\mathrm{M}}^{\text {no corr }}+k \rho_{\mathrm{M}}^{\text {corr }}=\rho_{\mathrm{M}}^{0} \text {. }
\end{aligned}
$$

Since in our heat engine, the initial state has no coherences, it suffices to consider $\rho_{\text {Coldmw }}^{1}$ which is diagonal in the energy eigenbasis. This implies that $\rho_{\text {ColdMW }}^{\text {no corr }}=\rho_{\text {ColdMW }}^{1}$ is also diagonal in the

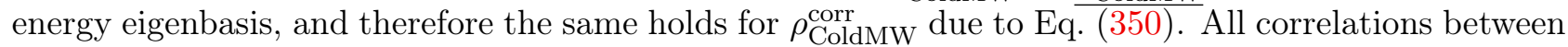
the individual systems of cold bath, machine and battery are contained only in $\rho_{\text {Coldmw }}^{\text {corr }}$. Therefore, $\rho_{\text {ColdMW }}^{1}(\cdot, \cdot, \cdot)$ parametrizes every possible quantum state on $\mathcal{H}_{\text {ColdMw }}$ which is diagonal in the global energy eigenbasis and that returns the machine locally to its initial state after one cycle of the heat engine. In Eq. (353), $\rho_{\mathrm{M}}^{1}$ is the final state of the machine, since the heat engine is cyclic, recall from Section A that we require $\rho_{\mathrm{M}}^{1}=\rho_{\mathrm{M}}^{0}$.

Lemma 20. For every family of states $\rho_{\text {ColdMW }}^{1}\left(k, \rho_{\text {ColdMW }}^{\text {no corr }}, \rho_{\text {ColdMW }}^{\text {corr }}\right)$ parametrized by $k$, (see Eqs. (350)-(353)), if the quantum efficiency $\eta_{1}^{\mathrm{qm}}$ defined in Eq. (316) of a quasi-static heat engine achieves the Carnot efficiency

$$
\eta_{1}^{\mathrm{qm}}\left(\rho_{\text {ColdMW }}^{1}\right)=1-\frac{\beta_{h}}{\beta_{c}},
$$

then the following conditions are satisfied:

1) The state $\rho_{\text {ColdMw }}^{1}$ is the final state of a quasi-static heat engine (see Def. 6)

$$
\underline{\rho_{\text {ColdMW }}^{1}}=\tau(g) \otimes \rho_{\mathrm{M}}^{0}(g) \otimes \rho_{\mathrm{W}}^{1} \quad \text { with } g \rightarrow 0^{+} .
$$

2) The correlations must vanish sufficiently quickly. That is to say, the parameter $k$ in Eq. (350) vanishes more quickly compared to g, i.e.

$$
\lim _{g \rightarrow 0^{+}} \frac{k}{g}=0
$$

Proof. Firstly, suppose that Carnot efficiency is achieved, i.e. $\eta^{\mathrm{qm}}\left(\rho_{\text {ColdMw }}^{1}\right)=1-\frac{\beta_{h}}{\beta_{c}}$. Then according to Lemma 19, all inequalities in Eq. (335) should be satisfied with equality, in particular inequality (4). We have established in Lemma 6 that this equality is achieved in the quasi-static limit, i.e. $\rho_{\text {Cold }}^{1}=\tau_{\text {Cold }}(g)$ where $g \rightarrow 0^{+}$. This implies Condition 1) in the statement of the lemma.

The proof for Condition 2) consists of calculating $W_{\text {ext }}$ for $\alpha=1$ in Eq. (316) to leading order in $g$ and $k$. This $W_{\text {ext }}$ quantity can be later used to evaluate $\eta_{1}^{\mathrm{qm}}$. We will show that we can write the expression for $\eta_{1}^{\mathrm{qm}}$ into two terms: one term describes the efficiency when there are no final correlations, and the other term is a strictly negative contribution which must vanish in order to achieve the Carnot efficiency. This latter constraint will give us Eq. (356).

Let us denote $W_{\text {ext }}^{\prime}$ as the value of battery energy gap $W_{\text {ext }}=E_{k}^{\mathrm{W}}-E_{j}^{\mathrm{W}}$ that solves the equation

$$
F_{1}\left(\tau_{\text {Cold }}^{0} \otimes \rho_{\mathrm{M}}^{0}(g) \otimes \rho_{\mathrm{W}}^{0}, \tau_{\text {ColdMW }}^{h}\right)=F_{1}\left(\rho_{\text {ColdMW }}^{1}\left(k, \rho_{\text {ColdMW }}^{\text {no corr }}, \rho_{\text {ColdMW }}^{\text {corr }}\right), \tau_{\text {ColdMW }}^{h}\right)^{16},
$$

while $\hat{W}_{\text {ext }}$ as the value that solves the case where $k=0$, i.e.

$$
F_{1}\left(\tau_{\text {Cold }}^{0} \otimes \rho_{\mathrm{M}}^{0}(g) \otimes \rho_{\mathrm{W}}^{0}, \tau_{\text {ColdMW }}^{h}\right)=F_{1}\left(\rho_{\text {ColdMW }}^{\text {no corr }}, \tau_{\text {ColdMW }}^{h}\right) .
$$

Since $\rho_{\text {ColdMW }}^{\text {no corr }}=\underline{\rho_{\text {ColdMW }}^{1}}=\rho_{\text {Cold }}^{1} \otimes \rho_{\mathrm{M}}^{0}(g) \otimes \rho_{\mathrm{W}}^{1}$ contains no correlations, $\hat{W}_{\text {ext }}$ was given by Eq. (126). According to Lemma 18, we know that $W_{\text {ext }}^{\prime}$ and $\hat{W}_{\text {ext }}$ are the values of $W_{\text {ext }}$ which solve

\footnotetext{
${ }^{16}$ We denote $\rho_{\mathrm{M}}^{0}(g)$ because for different values of $g$, we are allowed to choose different initial machine states, as long as $\rho_{\mathrm{M}}^{1}(g)=\rho_{\mathrm{M}}^{0}(g)$.
} 
$\sup _{W_{\text {ext }}} \eta_{1}^{\mathrm{qm}}\left(\rho_{\text {ColdMw }}^{1}, W_{\text {ext }}\right)$ and $\sup _{W_{\text {ext }}} \eta_{1}^{\mathrm{qm}}\left(\underline{\rho_{\text {ColdMw }}^{1}}, W_{\text {ext }}\right)$ respectively. Solving Eq. (357) for $W_{\text {ext }}^{\prime}$ with the aid of Eq. (328), we find

$$
W_{\text {ext }}^{\prime}=\hat{W}_{\text {ext }}-\chi
$$

where $W_{\text {ext }}$ is the solution to Eq. (358) when $k=0$, given by Eq. (126), while

$$
\chi:=\frac{1}{\beta_{h}} \frac{1}{1-\varepsilon}\left[S\left(\rho_{\text {ColdMW }}^{\text {no corr }}\right)-S\left(\rho_{\text {ColdMW }}^{1}\left(k, \rho_{\text {ColdMW }}^{\text {no corr }}, \rho_{\text {ColdMW }}^{\text {corr }}\right)\right)\right] .
$$

Let us first note some properties of $\chi$, which we will later use:

- Since $S(\cdot)$ is subadditive, due to the parametrization of $\rho_{\text {Coldmw }}^{1}(\cdot, \cdot, \cdot)$ in Eq. (350), we have

$$
\chi \geq 0
$$

with equality iff $\underline{\rho_{\text {ColdMW }}^{1}}=\rho_{\text {ColdMw }}^{1}$ i.e. iff $k=0$. Therefore, we may conclude that $\frac{\hat{W}_{\text {ext }}}{W_{\text {ext }}^{1}} \geq 1$.

- We have that

$$
\left.\frac{d}{d k} \chi\left(k, \rho_{\text {ColdMW }}^{\text {no corr }}, \rho_{\text {ColdMW }}^{\text {corr }}\right)\right|_{k=k_{0}}=0
$$

if and only if

$$
\rho_{\text {ColdMW }}^{1}\left(k_{0}, \rho_{\text {ColdMW }}^{\text {no corr }}, \rho_{\text {ColdMW }}^{\text {corr }}\right)=\mathbb{1}_{\text {ColdMW }} / N .
$$

Eqs. (362) and (363) are direct consequences of the observations:

1) Entropy is strictly concave, i.e. $S\left(\rho_{\text {ColdMW }}^{1}\left(k, \rho_{\text {ColdMW }}^{\text {no corr }}, \rho_{\text {ColdMW }}^{\text {corr }}\right)\right)$ is strictly concave in $k \in[0,1]$. Therefore, by Eq. (363) $\chi$ is strictly convex in $k \in[0,1]$. When the first derivative of the convex function $\frac{d \chi}{d k}=0$, this must be the global minimum [66].

2) However, we know that the entropy is uniquely maximized (and therefore $\chi$ is uniquely minimized) for the maximally mixed state.

Returning to evaluate the efficiency, we may use Eq. (315) to calculate the inverse efficiency,

$$
\begin{aligned}
{\left[\eta_{1}^{\mathrm{qm}}\left(\rho_{\text {ColdMw }}^{1}\right)\right]^{-1} } & =1-\varepsilon+\frac{\Delta C\left(\rho_{\text {Cold }}^{1}\right)}{W_{\text {ext }}^{\prime}} \\
& =1-\varepsilon+\frac{\Delta C\left(\rho_{\text {Cold }}^{1}\right)}{\hat{W}_{\text {ext }}} \frac{\hat{W}_{\text {ext }}}{W_{\text {ext }}^{\prime}} \\
& \geq 1-\varepsilon+\frac{\Delta C\left(\rho_{\text {Cold }}^{1}\right)}{\hat{W}_{\text {ext }}} .
\end{aligned}
$$

The last term in Eq. (365) is non-negative because we know the terms $\Delta C\left(\rho_{\text {Cold }}^{1}\right), \hat{W}_{\text {ext }}$ and $W_{\text {ext }}^{\prime}$ are all non-negative.

With Condition 1), we now know that

$$
1-\varepsilon+\frac{\Delta C\left(\rho_{\text {Cold }}^{1}\right)}{W_{\text {ext }}}=1-\frac{\beta_{h}}{\beta_{c}},
$$

in the quasi-static limit, and therefore a necessary condition to achieve the Carnot efficiency is that $\lim _{g \rightarrow 0} \frac{\hat{W}_{\text {ext }}}{W_{\text {ext }}^{\prime}}=1$ also in the quasi-static limit. Using the relation $W_{\text {ext }}^{\prime}=\hat{W}_{\text {ext }}+\chi$, we have the requirement that

$$
\lim _{g \rightarrow 0^{+}} \frac{\chi\left(k, \rho_{\text {ColdMW }}^{\text {no corr }}(g), \rho_{\text {ColdMW }}^{\text {corr }}\right)}{\hat{W}_{\text {ext }}\left(\rho_{\text {ColdMW }}^{\text {norr }}(g)\right)}=0 .
$$

First, let us observe that $\hat{W}_{\text {ext }}\left(\rho_{\text {ColdMW }}^{\text {no corr }}(g)\right)=W_{\text {ext }}\left(\beta_{c}-g\right)$ given by Eq. (113). The leading order term of $W_{\text {ext }}\left(\beta_{c}-g\right)=\Theta(g)$ as $g \rightarrow 0^{+}$. Therefore, in order to satisfy Eq. (368), we must firstly have $\lim _{g \rightarrow 0} \chi=0$. From Eqs. (350), (360), this implies that we need $k \rightarrow 0$ for all $\rho_{\text {ColdMW }}^{\text {no corr }}$. 
Since the numerator and denominator of Eq. (368) both go to zero, by L'Hospital rule, to evaluate the limit we need to take the derivative of both terms w.r.t. $g$. Therefore, we expand $\chi$ to first order in $k$ and $g$. From Eq. (360) it follows

$$
\begin{aligned}
\chi\left(k, \rho_{\text {ColdMW }}^{\text {no corr }}(g), \rho_{\text {ColdMW }}^{\text {corr }}\right)= & \left.\frac{d}{d k} \chi\left(k, \rho_{\text {ColdMW }}^{\text {no corr }}(0), \rho_{\text {ColdMW }}^{\text {corr }}\right)\right|_{k=0} k \\
& +\left.\frac{d}{d g} \chi\left(0, \rho_{\text {ColdMW }}^{\text {no corr }}(g), \rho_{\text {ColdMW }}^{\text {corr }}\right)\right|_{g=0} g+o(g k)+o\left(k^{2}\right)+o\left(g^{2}\right) \\
= & \left.\frac{d}{d k} \chi\left(k, \rho_{\text {ColdMW }}^{\text {no corr }}(0), \rho_{\text {ColdMW }}^{\text {corr }}\right)\right|_{k=0} k+o(g k)+o\left(k^{2}\right)+o\left(g^{2}\right) .
\end{aligned}
$$

The term $\left.\frac{d}{d g} \chi\left(0, \rho_{\text {ColdMW }}^{\text {no corr }}(g), \rho_{\text {ColdMW }}^{\text {corr }}\right)\right|_{g=0}=0$ since when $k=0, \chi$ will be constant for all $g$. Next, we note that since Eqs. (361) holds, it must be that $\left.\frac{d}{d k} \chi\left(k, \rho_{\text {ColdMW }}^{\text {no corr }}(0), \rho_{\text {ColdMW }}^{\text {corr }}\right)\right|_{k=0} \geq 0$. Furthermore, from Eq. (362), we have that

$$
\left.\frac{d}{d k} \chi\left(k, \rho_{\text {ColdMW }}^{\text {no corr }}(0), \rho_{\text {ColdMW }}^{\text {corr }}\right)\right|_{k=0} \neq 0,
$$

for all $\rho_{\text {ColdMW }}^{\text {corr }}$ since by definition $\rho_{\text {ColdMW }}^{1}\left(0, \rho_{\text {ColdMW }}^{\text {no corr }}(0), \rho_{\text {ColdMW }}^{\text {corr }}\right) \neq \mathbb{1}_{\text {ColdMW }} / N$. We can infer that $\rho_{\text {ColdMw }}^{1}$ is not maximally mixed from a few observations, for example: this is true because we have required that the reduced state on the battery is not maximally mixed since we consider near perfect work extraction.

Thus, taking into account $W_{\text {ext }}\left(\beta_{c}-g\right)=\Theta(g)$, Eq. (368) implies Eq. (356).

By now, we have established a constraint on how quickly the correlations have to vanish w.r.t. $g$, for the possibility of achieving Carnot efficiency. In the next Lemma 21, we will show that the constraints given by Eq. (356) can be used to derive an upper bound for $\eta_{\infty}^{\mathrm{qm}}$.

Lemma 21. If Eqs. (355) and (356) are satisfied, then the quantity $\eta_{\infty}^{\mathrm{qm}}$ can be upper bounded by

$$
\begin{aligned}
& \eta_{\infty}^{\mathrm{qm}}\left(\rho_{\text {ColdMW }}^{1}\left(k, \rho_{\text {ColdMW }}^{\text {no corr }}(g), \rho_{\text {ColdMw }}^{\text {corr }}\right)\right) \\
& \leq\left[1+\frac{\beta_{h}}{\beta_{c}-\beta_{h}} \frac{\gamma(1)}{\gamma(\infty)}\right]^{-1}+\Theta(f(g))+\Theta(k / g)+\Theta(g)+\Theta(\varepsilon),
\end{aligned}
$$

with $\lim _{g \rightarrow 0^{+}} f(g)=0$.

Proof. The main idea of our proof is as follows: we show that if Eqns. (355) and (356) hold, then we can upper bound $W_{\text {ext }}$ while considering only the $F_{\infty}$ condition. This bound differs from the value given when no correlations are present by only a small amount. Substituting this into the expression for $\eta_{\infty}^{\mathrm{qm}}$, we obtain Eq. (373).

Let us begin by analyzing the difference in eigenvalues of the states $\rho_{\text {ColdMw }}^{1}$ and $\rho_{\text {ColdMw }}^{1}$. Recall that

$$
\rho_{\text {ColdMW }}^{1}\left(k, \rho_{\text {ColdMW }}^{\text {no corr }}, \rho_{\text {ColdMW }}^{\text {corr }}\right)=(1-k) \rho_{\text {ColdMW }}^{\text {no corr }}+k \rho_{\text {ColdMW }}^{\text {corr }}
$$

where $\rho_{\text {ColdMW }}^{\text {no corr }}, \rho_{\text {ColdMw }}^{\text {corr }}$ are both diagonal in the energy eigenbasis. Since $\rho_{\text {ColdMw }}^{1}$ is a mixture of two energy-diagonal states, it is also diagonal. Let us denote its eigenvalues as $\left[\rho_{\text {ColdMw }}^{1}\right]_{i}$.

As for $\rho_{\text {ColdMw }}^{1}$, Eqn. (355) gives the explicit form of the state,

$$
\underline{\rho_{\text {ColdMW }}^{1}}=\rho_{\text {Cold }}^{1} \otimes \rho_{\mathrm{M}}^{1} \otimes \rho_{\mathrm{W}}^{1}=\tau(g) \otimes \rho_{\mathrm{M}}^{0}(g) \otimes \rho_{\mathrm{W}}^{1} .
$$

Let us denote its eigenvalues as $\left[\rho_{\text {ColdMw }}^{1}\right]$.

We first observe two properties involving trace distance $d(\cdot, \cdot)$ : 
(P.i) Consider two states $\sigma_{1}, \sigma_{2}$ diagonal in the same eigenbasis. Then if $\rho=(1-k) \sigma_{1}+k \sigma_{2}$ for some $k \in[0,1]$, then one can conclude that the distance

$$
d\left(\rho, \sigma_{1}\right) \leq k
$$

(P.ii) For any two states $\rho, \sigma$ diagonal in the same basis, with eigenvalues $p_{i}, q_{i}$, if their trace distance

$$
d(\rho, \sigma)=\frac{1}{2}\|\rho-\sigma\|_{1} \leq \varepsilon,
$$

then this implies that their eigenvalues cannot differ by more than $\varepsilon$, i.e. $\forall i,\left|p_{i}-q_{i}\right| \leq \varepsilon$. By using this fact, we may first calculate the trace distance between $\rho_{\text {ColdMw }}^{1}$ and $\rho_{\text {ColdMw }}^{1}$, then bound the difference of their eigenvalues.

We find that

$$
\begin{aligned}
d\left(\rho_{\text {ColdMW }}^{1}, \underline{\rho_{\text {ColdMW }}^{1}}\right) & \leq d\left(\rho_{\text {ColdMW }}^{1}, \rho_{\text {ColdMW }}^{\text {no corr }}\right)+d\left(\rho_{\text {ColdMW }}^{\text {no corr }}, \rho_{\text {ColdMW }}^{1}\right) \\
& \leq k+d\left(\rho_{\text {Cold }}^{\text {no corr }}, \rho_{\text {Cold }}^{1}\right)+d\left(\rho_{\mathrm{M}}^{\text {no corr }}, \rho_{\mathrm{M}}^{1}\right)+d\left(\rho_{\mathrm{W}}^{\text {no corr }}, \rho_{\mathrm{W}}^{1}\right) \\
& \leq 4 k .
\end{aligned}
$$

The first inequality is a triangle inequality that holds for all states. The second inequality holds because of (P.i), and because trace distance is subadditive under tensor product (note that both $\rho_{\text {ColdMW }}^{\text {no corr }}$ and $\rho_{\text {ColdMw }}^{1}$ are tensor product states). The third inequality holds because we know $d\left(\rho_{\text {ColdMW }}^{1}, \rho_{\text {ColdMW }}^{\text {no }}\right) \leq k$ and that trace distance decreases under partial trace. By (P.ii), Eq. (380) tells us that $\forall i$,

$$
\left[\rho_{\text {ColdMW }}^{1}\right]_{i}=\left[\underline{\rho_{\text {ColdMW }}^{1}}\right]_{i}+o(k) .
$$

With Eq.(381), we may relate the $F_{\infty}$ quantities for the states $\rho_{\text {ColdMw }}^{1}$ and $\rho_{\text {ColdMw }}^{1}$. From Eq. (56), we have

$$
\begin{aligned}
F_{\infty}\left(\rho _ { \text { ColdMW } } ^ { 1 } \left(k, \rho_{\text {ColdMW }}^{\text {no corr }}(g)\right.\right. & \left.\left., \rho_{\text {ColdMw }}^{\text {corr }}\right), \tau_{\text {ColdMW }}^{h}\right) \\
& =\ln \max _{i}\left\{\frac{\left[\rho_{\text {ColdMW }}^{1}\right]_{i}}{\tau_{i}}\right\} \\
& =\ln \max _{i}\left\{\frac{\left[\rho_{\text {ColdMW }}^{1}\right]_{i}}{\tau_{i}}\right\}+o(k), \\
& =F_{\infty}\left(\tau(g) \otimes \rho_{\mathrm{M}}^{0}(g) \otimes \rho_{\mathrm{W}}^{1}, \tau_{\text {ColdMW }}^{h}\right)+o(k),
\end{aligned}
$$

where we used Eq. (56) in the last line.

The next step is to evaluate the restriction on $W_{\text {ext }}$ that satisfies

$$
\begin{aligned}
F_{\infty}\left(\tau_{\text {Cold }}^{0} \otimes \rho_{\mathrm{M}}^{0} \otimes \rho_{\mathrm{W}}^{0}, \tau_{\text {ColdMW }}^{h}\right) & \geq F_{\infty}\left(\rho_{\text {ColdMW }}^{1}\left(k, \rho_{\text {ColdMW }}^{\text {no corr }}(g), \rho_{\text {ColdMW }}^{\text {corr }}\right), \tau_{\text {ColdMW }}^{h}\right) \\
& =F_{\infty}\left(\tau(g) \otimes \rho_{\mathrm{M}}^{0}(g) \otimes \rho_{\mathrm{W}}^{1}, \tau_{\text {ColdMW }}^{h}\right)+o(k),
\end{aligned}
$$

for $W_{\text {ext }}$ up to order $o(k)$. Taking into account the additivity of $F_{\infty}$ under tensor product, we can rearrange Eq. (387) to provide an upper bound on $W_{\text {ext }}$,

$$
W_{\text {ext }} \leq \frac{n g}{\beta_{h}}[\gamma(\infty)+\Theta(f(g))+o(k / g)],
$$

where $\lim _{g \rightarrow 0^{+}} f(g)=0, \gamma(\infty)$ is given by Eq. (276). The bound in Eq. (388) is achievable since the $F_{\infty}$ conditions imposed by Eq. (386) are achievable with equality. 
Lastly, by using the expression for efficiency in Eqs. (315), and substituting $W_{\text {ext }}$ from Eq. (388) (with equality for the maximum possible $W_{\text {ext }}$ ) followed by $\Delta C$ from Eq. (279), we have

$$
\begin{aligned}
\sup _{W_{\text {ext }}>0} \eta\left(\rho_{\text {Cold }}^{1}, W_{\text {ext }}\right) & =\sup _{W_{\text {ext }}>0}\left(1-\varepsilon+\frac{\Delta C}{W_{\text {ext }}}\right)^{-1} \\
& =\left[1+\frac{\beta_{h}}{\left(\beta_{c}-\beta_{h}\right)} \frac{\gamma(1)}{\gamma(\infty)}\right]^{-1}+\Theta(f(g))+o(k / g)+\Theta(g)+\Theta(\varepsilon) .
\end{aligned}
$$

Hence using Eqs. (316), (390) we find Eq. (373). Note that in Eq (373) we have an inequality, this is due to the fact that in the optimisation problem Eq. (316), there is an additional constraint (namely mean energy conservation) which is not taken into account in the derivation of Eq. (390).

Finally, the above lemmas allow us to conclude that allowing further correlations in the final state cannot allow quasi-static heat engines to achieve the Carnot efficiency when $\Omega>1$.

Theorem 3. [Correlations do not improve efficiency] Suppose that $\Omega>1$. Parametrizing the final state of the heat engine by Eq. (350)-(353), the quantum efficiency $\eta^{\mathrm{qm}}$ defined in Eq. (311) in a quasi-static heat engine is strictly upper bounded by the Carnot efficiency,

$$
\sup _{k \in[0,1], \rho_{\text {ColdMW }}^{\text {no corr }}} \eta^{\mathrm{qm}}\left(\rho_{\text {ColdMW }}^{1}\left(k, \rho_{\text {ColdMW }}^{\text {no corr }}, \rho_{\text {ColdMW }}^{\text {corr }}\right)\right)<1-\frac{\beta_{h}}{\beta_{c}} .
$$

Proof. From Lemma 17, we have that both $\eta^{\mathrm{qm}} \leq \eta_{1}^{\mathrm{qm}}$ and $\eta^{\mathrm{qm}} \leq \eta_{\infty}^{\mathrm{qm}}$ hold. Thus a necessary

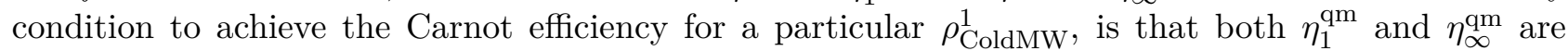
equal to or greater than the Carnot efficiency.

Lemma 20 proves that Eqs. (355) and (356) are necessary conditions for $\eta_{1}^{\mathrm{qm}}$ to achieve the Carnot efficiency. However, when Eqs. (355), (356) are satisfied, then Lemma 21 provides an upper bound on the efficiency $\eta_{\infty}^{\mathrm{qm}}$ in Eq. (373).

Now, suppose $\Omega>1$. Since it is shown in Eq. (277) that $\gamma(1) / \gamma(\infty)=\Omega$, plugging this into the leading term appearing in Eq. (373)

$$
\left[1+\frac{\beta_{h}}{\left(\beta_{c}-\beta_{h}\right)} \frac{\gamma(1)}{\gamma(\infty)}\right]^{-1}
$$

we have that the quantity $\eta_{\infty}^{\mathrm{qm}}$ (and therefore also $\eta^{\mathrm{qm}}$ ) is strictly less than the Carnot efficiency $1-\beta_{h} / \beta_{c}$.

\section{F.2 A more general final battery state}

For the simplicity of our analysis, we have assumed that the battery is left in the specific final state described in Eq. (41), i.e. an amount of work $W_{\text {ext }}=E_{k}-E_{j}$ is extracted, except with failure probability $\varepsilon$ that the battery remains in the initial state $\left|E_{j}\right\rangle\left\langle\left. E_{j}\right|_{\mathrm{W}}\right.$. In this section, we show that this is a simplification which can be removed in general, i.e. the final battery state is allowed to be any state within the $\varepsilon$-ball of $\left|E_{k}\right\rangle\left\langle\left. E_{k}\right|_{\mathrm{W}}\right.$. In particular, our result that the Carnot efficiency cannot be achieved when $\Omega>1$ still holds.

In Lemma 22, we show that for any final state of the cold bath $\rho_{\text {Cold }}^{1}$, allowing a more general final battery state does not affect the amount of work bounded by the $F_{\infty}$ condition. We then use this to prove in Theorem 4 that when $\Omega>1$, the Carnot efficiency cannot be achieved even if we allow a more general battery final state.

Lemma 22. For any given $\rho_{\text {Cold }}^{0}, \rho_{\text {Cold }}^{1}$, with $\rho_{\mathrm{W}}^{0}=\left|E_{j}\right\rangle\left\langle\left. E_{j}\right|_{\mathrm{W}}\right.$, consider the maximum $W_{\infty}^{1}:=E_{k_{1}}-E_{j}$ such that $\rho_{\text {Cold }}^{0} \otimes \rho_{\mathrm{W}}^{0} \rightarrow \rho_{\text {Cold }}^{1} \otimes \rho_{\mathrm{W}}^{1}$ is allowed by the non-increasing $F_{\infty}$ condition (Eq. (55)) i.e.

$$
D_{\infty}\left(\rho_{\text {Cold }}^{0} \| \tau_{\text {Cold }}^{\beta_{h}}\right)+D_{\infty}\left(\rho_{\mathrm{W}}^{0} \| \tau_{\mathrm{W}}^{\beta_{h}}\right) \geq D_{\infty}\left(\rho_{\text {Cold }}^{1} \| \tau_{\text {Cold }}^{\beta_{h}}\right)+D_{\infty}\left(\rho_{\mathrm{W}}^{1} \| \tau_{\mathrm{W}}^{\beta_{h}}\right),
$$


with

$$
\rho_{\mathrm{W}}^{1}=(1-\varepsilon)\left|E_{k_{1}}\right\rangle\left\langle\left. E_{k_{1}}\right|_{\mathrm{W}}+\varepsilon \mid E_{j}\right\rangle\left\langle\left. E_{j}\right|_{\mathrm{W}} .\right.
$$

On the other hand, consider any battery final state

$$
\rho_{\mathrm{W}}^{2}=(1-\varepsilon)\left|E_{k_{2}}\right\rangle\left\langle\left. E_{k_{2}}\right|_{\mathrm{W}}+\varepsilon \rho_{\mathrm{W}}^{\mathrm{junk}},\right.
$$

where $\rho_{\mathrm{W}}^{\mathrm{junk}}$ is an energy-diagonal state orthogonal to $\left|E_{k_{2}}\right\rangle\left\langle\left. E_{k_{2}}\right|_{\mathrm{W}}\right.$ which may depend on $\varepsilon$, i.e. $\rho_{\mathrm{W}}^{\mathrm{junk}}=$ $\sum_{i} p_{i}\left|E_{i}\right\rangle\left\langle\left. E_{i}\right|_{\mathrm{W}}\right.$ with $p_{k_{2}}=0$ and $\sum_{i} p_{i}=1$. Define $W_{\infty}^{2}:=E_{k_{2}}-E_{j}$ such that $\rho_{\text {Cold }}^{0} \otimes \rho_{\mathrm{W}}^{0} \rightarrow \rho_{\text {Cold }}^{1} \otimes \rho_{\mathrm{W}}^{2}$ is allowed by the non-increasing $F_{\infty}$ condition, i.e.

$$
D_{\infty}\left(\rho_{\text {Cold }}^{0} \| \tau_{\text {Cold }}^{\beta_{h}}\right)+D_{\infty}\left(\rho_{\mathrm{W}}^{0} \| \tau_{\mathrm{W}}^{\beta_{h}}\right) \geq D_{\infty}\left(\rho_{\text {Cold }}^{1} \| \tau_{\text {Cold }}^{\beta_{h}}\right)+D_{\infty}\left(\rho_{\mathrm{W}}^{2} \| \tau_{\mathrm{W}}^{\beta_{h}}\right) .
$$

Then for all $0<\varepsilon \leq \hat{\varepsilon}=\left[1+e^{\beta_{h}\left(E_{\max }-E_{j}\right)}\right]^{-1}$, we have $W_{\infty}^{1}=W_{\infty}^{2}$.

Proof. Firstly, note that any energy-diagonal state $\rho_{\mathrm{W}}^{2}$ with trace distance $d\left(\rho_{\mathrm{W}}^{2},\left|E_{k_{2}}\right\rangle\left\langle\left. E_{k_{2}}\right|_{\mathrm{W}}\right)=\varepsilon\right.$ can be written in the form of Eq. (395). Rearranging the terms in Eq. (393),

$$
D_{\infty}\left(\rho_{\mathrm{W}}^{1} \| \tau_{\mathrm{W}}^{\beta_{h}}\right) \leq D_{\infty}\left(\rho_{\mathrm{W}}^{0} \| \tau_{\mathrm{W}}^{\beta_{h}}\right)+D_{\infty}\left(\rho_{\text {Cold }}^{0} \| \tau_{\text {Cold }}^{\beta_{h}}\right)-D_{\infty}\left(\rho_{\text {Cold }}^{1} \| \tau_{\text {Cold }}^{\beta_{h}}\right)=: A .
$$

One can use the definition of $D_{\infty}$ in Eq. (60) to expand the L.H.S. of Eq. (397), obtaining

$$
\log \max \left\{(1-\varepsilon) e^{\beta_{h} E_{k_{1}}}, \varepsilon e^{\beta_{h} E_{j}}\right\} \leq A-\log Z_{\mathrm{W}}^{\beta_{h}} .
$$

We know that since near perfect work is extracted, $\varepsilon$ is arbitrarily small. This implies that for $\varepsilon$ small enough, $\max \left\{(1-\varepsilon) e^{\beta_{h} E_{k_{1}}}, \varepsilon e^{\beta_{h} E_{j}}\right\}=(1-\varepsilon) e^{\beta_{h} E_{k_{1}}}$.

Similarly, one can evaluate Eq. (393) to obtain

$$
\log \max \left\{(1-\varepsilon) e^{\beta_{h} E_{k_{2}}},\left\{\varepsilon p_{i} e^{\beta_{h} E_{i}}\right\}_{i \neq k_{2}}\right\} \leq A-\log Z_{\mathrm{W}}^{\beta_{h}} .
$$

Note that the maximization in Eq. (399) only picks out the maximum value. In particular, denoting $E_{\max }$ to be the largest energy eigenvalue of the battery, then whenever

$$
(1-\varepsilon) e^{\beta_{h} E_{k_{2}}} \geq \varepsilon e^{\beta_{h} E_{\max }},
$$

or equivalently

$$
\varepsilon \leq\left[1+e^{\beta_{h}\left(E_{\max }-E_{k_{2}}\right)}\right]^{-1},
$$

then $\max \left\{(1-\varepsilon) e^{\beta_{h} E_{k_{2}}},\left\{\varepsilon p_{i} e^{\beta_{h} E_{i}}\right\}_{i \neq k_{2}}\right\}=(1-\varepsilon) e^{\beta_{h} E_{k_{2}}}$. In other words, as long as $\varepsilon$ is upper bounded by Eq. (401), we know which terms attains the maximization in Eq. (398). However, we also want an upper bound that is independent of any limit involving the final state $\rho_{\text {ColdMw }}^{1}$ we wish to take, or any amount of work extracted (and therefore, we want the bound to be independent of $E_{k_{2}}$ ). As such, let us construct the following upper bound $\varepsilon \leq \hat{\varepsilon}$ where,

$$
\hat{\varepsilon}:=\inf _{E_{k_{2}}}\left[1+e^{\beta_{h}\left(E_{\max }-E_{k_{2}}\right)}\right]^{-1}=\left[1+e^{\beta_{h}\left(E_{\max }-E_{j}\right)}\right]^{-1}
$$

Now, we see that $E_{k_{1}}$ and $E_{k_{2}}$ correspond to the solutions for Eq. (398) and Eq. (399), which for $\varepsilon \leq \hat{\varepsilon}$ reduce to exactly the same equation. Therefore, $E_{k_{1}}=E_{k_{2}}$ and hence $W_{\infty}^{1}=W_{\infty}^{2}$.

We will use Lemma 22 to prove Theorem 4. But before we proceed, let us fix some notation: we define the efficiency as a function of $\alpha \geq 0$ :

$$
\eta_{\alpha}^{J}\left(\rho_{\text {Cold }}^{1}\right)=\sup _{E_{k_{J}}-E_{j}>0} \eta\left(\rho_{\text {Cold }}^{1}\right) \quad \text { subject to } \quad F_{\alpha}\left(\rho_{\mathrm{W}}^{0} \otimes \tau_{\text {Cold }}^{0}, \tau_{\text {ColdW }}^{h}\right) \geq F_{\alpha}\left(\rho_{\mathrm{W}}^{J} \otimes \rho_{\text {Cold }}^{1}, \tau_{\text {ColdW }}^{h}\right),
$$

$$
\text { and } \operatorname{tr}\left(\hat{H}_{t} \rho_{\text {ColdHotMW }}^{0}\right)=\operatorname{tr}\left(\hat{H}_{t} \rho_{\text {ColdHotMW }}^{1, J}\right)
$$


with $J=1,2$ denoting the final battery state $\rho_{\mathrm{W}}^{J}$. We also define an $\alpha$ independent efficiency:

$$
\begin{array}{ll}
\eta^{J}\left(\rho_{\text {Cold }}^{1}\right)=\sup _{E_{k_{J}}-E_{j}>0} \eta\left(\rho_{\text {Cold }}^{1}\right) \quad \text { subject to } \\
\quad F_{\alpha}\left(\rho_{\mathrm{W}}^{0} \otimes \tau_{\text {Cold }}^{0}, \tau_{\text {ColdW }}^{h}\right) \geq F_{\alpha}\left(\rho_{\mathrm{W}}^{J} \otimes \rho_{\text {Cold }}^{1}, \tau_{\text {ColdW }}^{h}\right) \forall \alpha \geq 0 .
\end{array}
$$

For any $\alpha \geq 0$, and any state $\rho_{\text {Cold }}^{1}, \eta_{\alpha}^{J}\left(\rho_{\text {Cold }}^{1}\right) \geq \eta^{J}\left(\rho_{\text {Cold }}^{1}\right)$ holds.

We already know that when $\Omega>1$, for any final cold bath state $\rho_{\text {Cold }}^{1}$, the efficiency $\eta^{1}\left(\rho_{\text {Cold }}^{1}\right)$ is strictly less than the Carnot efficiency. Theorem 4 shows that this is also true for $\eta^{2}\left(\rho_{\text {Cold }}^{1}\right)$, i.e. when allowing a more general battery final state.

Theorem 4. [General battery states do not improve efficiency] Consider a quasi-static heat engine with a cold bath consisting of $n$ qubits, extracting near perfect work. Let $\Omega>1$ (definition in Eq. (269)). Then the efficiency $\lim _{g \rightarrow 0^{+}} \eta^{2}\left(\tau_{\beta_{f}}\right)$, where $\tau_{\beta_{f}}$ is the final state of a quasi-static heat engine (Def. 6), is strictly less than the Carnot efficiency.

Proof. Firstly, suppose that $\Omega>1$. By Lemma 15 we know that the infimum is obtained at $\alpha=\infty$, and by Lemma 16 we know that the efficiency for quasi-static heat engine is strictly less than the Carnot value:

$$
\lim _{g \rightarrow 0^{+}} \eta^{1}\left(\tau_{\beta_{f}}\right)=\lim _{g \rightarrow 0^{+}} \eta_{\infty}^{1}\left(\tau_{\beta_{f}}\right)<\eta_{C}
$$

In other words, for all other final states $\rho_{\text {Cold }}^{1}$ we know that Carnot efficiency cannot be achieved. Therefore, it suffices to see that in the quasi-static limit,

$$
\lim _{g \rightarrow 0^{+}} \eta^{2}\left(\tau_{\beta_{f}}\right) \leq \lim _{g \rightarrow 0^{+}} \eta_{\infty}^{2}\left(\tau_{\beta_{f}}\right)=\lim _{g \rightarrow 0^{+}} \eta_{\infty}^{1}\left(\tau_{\beta_{f}}\right)=\lim _{g \rightarrow 0^{+}} \eta^{1}\left(\tau_{\beta_{f}}\right)<\eta_{C}
$$

The second equality is obtained by noting that for any state $\tilde{\rho}_{\text {Cold }}^{1}$ (and therefore for $\tau_{\beta_{f}}$ ):

1. $\Delta C$ is the same for both expressions of efficiency $\eta_{\infty}^{1}\left(\rho_{\text {Cold }}^{1}\right)$ and $\eta_{\infty}^{2}\left(\rho_{\text {Cold }}^{1}\right)$.

2. By Lemma 22 , for all $0<\varepsilon<\left[1+e^{\beta_{h}\left(E_{\max }-E_{j}\right)}\right]^{-1}, W_{\infty}^{1}\left(\tilde{\rho}_{\text {Cold }}^{1}\right)=W_{\infty}^{2}\left(\tilde{\rho}_{\text {Cold }}^{1}\right)$.

Hence, from Items 1 and 2 , one concludes that $\eta_{\infty}^{1}\left(\tilde{\rho}_{\text {Cold }}^{1}\right)=\eta_{\infty}^{2}\left(\tilde{\rho}_{\text {Cold }}^{1}\right)$. The third equality in Eq. (408) comes directly from Eq. (407). 